\title{
Traceless Staudinger Ligation to introduce chemical diversity on $\beta$-lactamase inhibitors of second generation
}

Flavie Bouchet, ${ }^{\dagger}$ Heiner Atze, ${ }^{\ddagger}$ Michel Arthur, ${ }^{\ddagger}$ Mélanie Ethève-Quelquejeu, ${ }^{* \dagger}$ Laura lannazzo* ${ }^{+}$

' Université de Paris, UMR CNRS 8601, Laboratoire de Chimie et Biochimie Pharmacologiques et Toxicologiques, F-75006 Paris, France.

‡ INSERM, Sorbonne Université, Université de Paris, Centre de Recherche des Cordeliers (CRC), F-75006 Paris, France.

laura.iannazzo@parisdescartes.fr

melanie.etheve-quelquejeu@parisdescartes.fr 


\section{Table of contents}

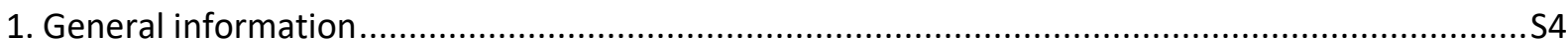

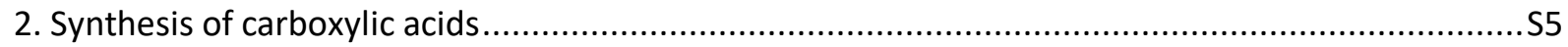

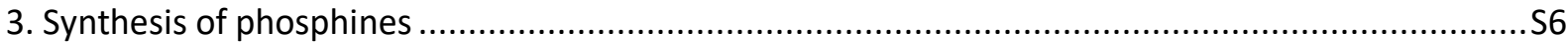

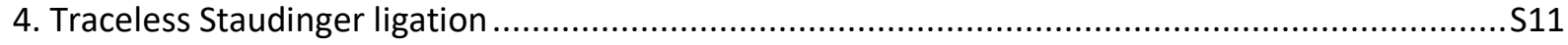

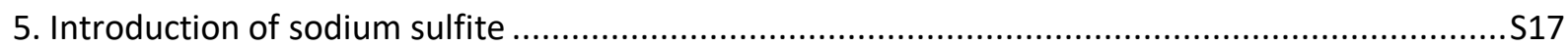

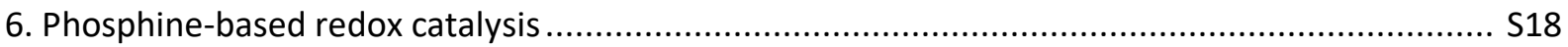

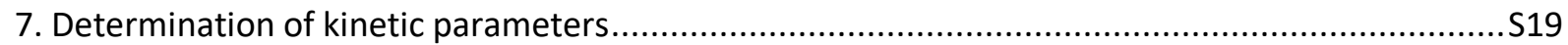

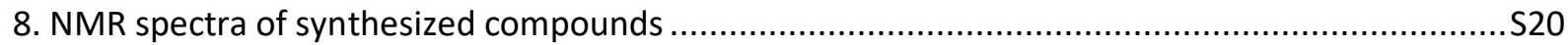

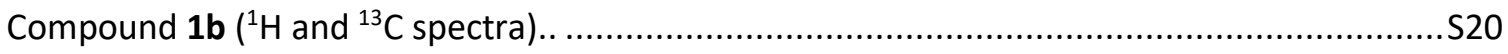

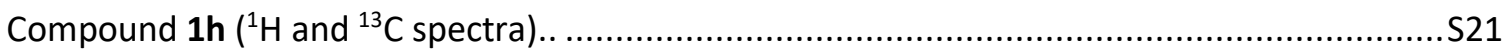

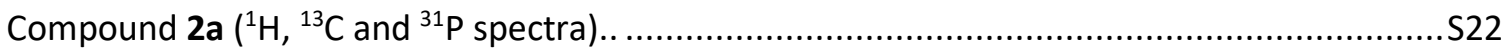

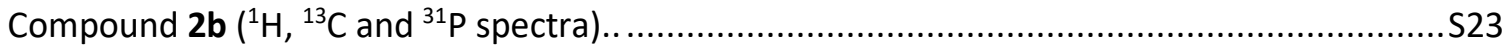

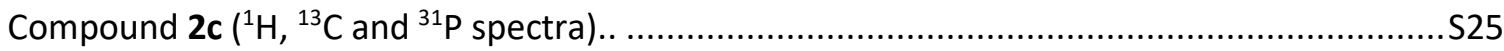

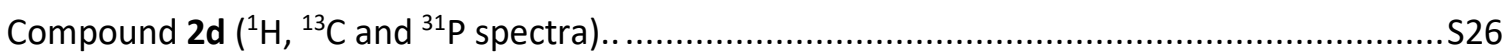

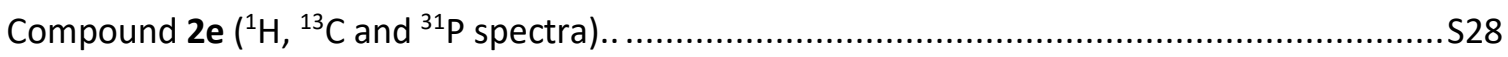

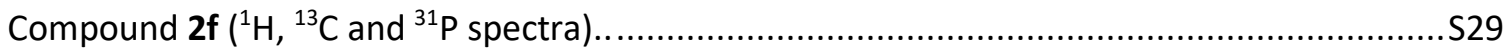

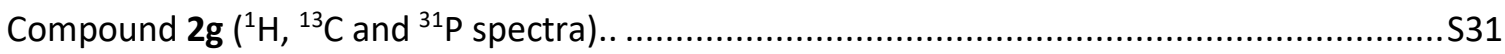

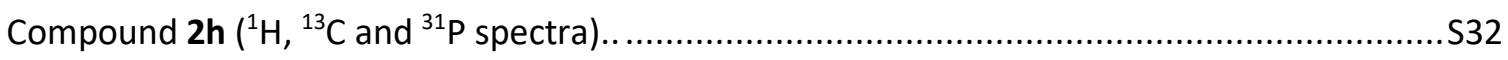

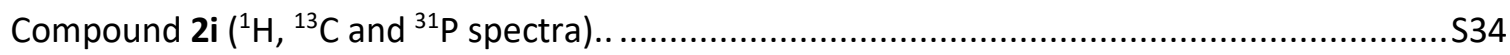

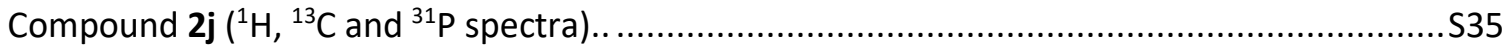

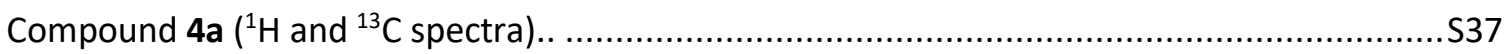

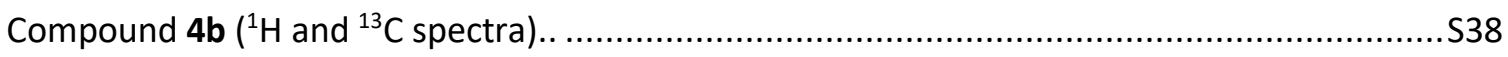

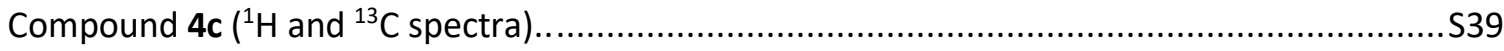


Compound $4 \mathbf{d}\left({ }^{1} \mathrm{H}\right.$ and ${ }^{13} \mathrm{C}$ spectra)..

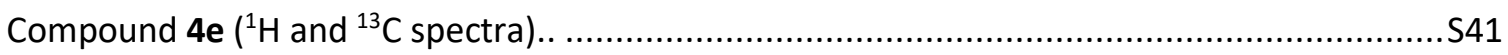

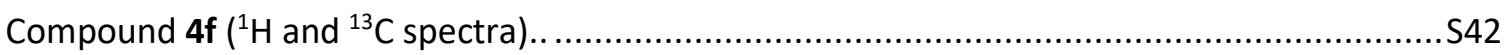

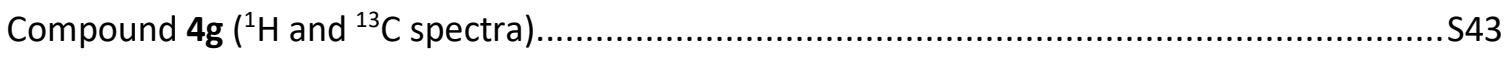

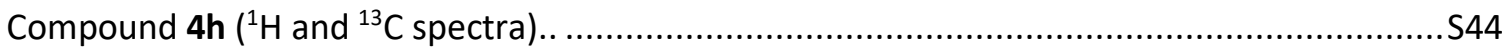

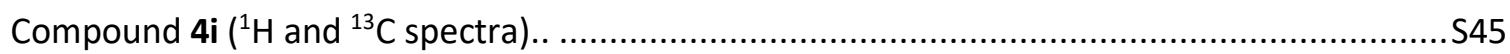

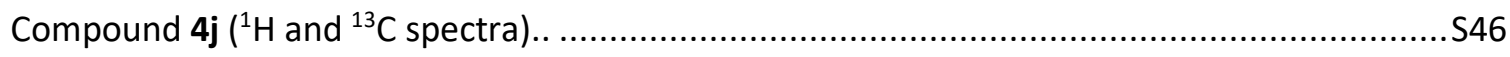

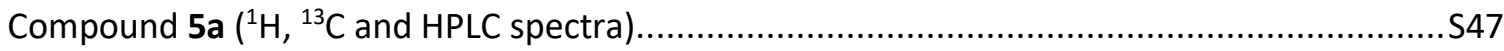




\section{General information}

\section{Synthesis}

Reactions were carried out under an argon atmosphere and solvents were dried using standard methods and distilled before use. Unless otherwise specified, materials were purchased from commercial suppliers and used without further purification. TLC was performed using Merck commercial aluminum sheets coated with silica gel $60 \mathrm{~F}_{254}$. Compounds were detected by charring with phosphomolibdic acid in ethanol followed by heating or by UV light at $254 \mathrm{~nm}$.

\section{Purification}

- Flash chromatography was done on silica gel (60 ^, 180-240 mesh) from Merck.

- Preparative high-performance liquid chromatography (HPLC) was performed using a Shimadzu Prominence system with a Zorbax Extend-C18 prepHT column (150 x $21.2 \mathrm{~mm}, 5 \mu \mathrm{m})$ from Agilent. Compounds were eluted with a linear gradient (from $100 \% \mathrm{H}_{2} \mathrm{O}$ to $100 \% \mathrm{CH}_{3} \mathrm{CN}$ ) that was applied between 5 and $30 \mathrm{~min}$ at a flow rate of $15 \mathrm{~mL} / \mathrm{min}$. Products were detected by UV absorption at $214 \mathrm{~nm}$.

\section{Analysis}

- NMR spectra were recorded on Bruker spectrometers (Avance II 500 and Avance III HD 4000). Chemical shifts $(\delta)$ are reported in parts per million (ppm) and referenced to the residual proton or carbon resonance of the solvents: $\mathrm{CDCl}_{3}(\delta 7.26)$ or $\mathrm{D}_{2} \mathrm{O}(\delta 4.79)$ for ${ }^{1} \mathrm{H}$ and $\mathrm{CDCl}_{3}(\delta$ 77.16) for ${ }^{13} \mathrm{C}$. Signals were assigned using $1 \mathrm{D}\left({ }^{1} \mathrm{H}\right.$ and $\left.{ }^{13} \mathrm{C}\right)$ and $2 \mathrm{D}(\mathrm{HSQC}, \mathrm{COSY}$ and $\mathrm{HMBC})$ spectroscopy. NMR coupling constants $(J)$ are reported in hertz $(\mathrm{Hz})$.

- High-resolution mass spectroscopy (HRMS) was performed with an ion trap mass analyser under electrospray ionization (ESI) in the negative or positive ionization detection mode. HRMS was performed using Thermo Scientific LTQ Orbitrap XL.

- The purity of final compound ( $\geq 95 \%$ ) was established by analytical HPLC, which was performed on a Shimadzu Prominence system with a Hypersil ${ }^{\circledR}$ BDS C18 column $(150 \times 4.6 \mathrm{~mm}, 5 \mu \mathrm{m})$ with UV detection at $220 \mathrm{~nm}$.

- Optical rotations were measured with a sodium lamp $(589 \mathrm{~nm})$ at $20^{\circ} \mathrm{C}$ on a Perkin Elmer polarimeter. 


\section{Synthesis of carboxylic acids}

Carboxylic acids $\mathbf{1 a}, \mathbf{1 c}, \mathbf{1 d}, \mathbf{1 e}, \mathbf{1 f}, \mathbf{1} \mathbf{g}, \mathbf{1}$ and $\mathbf{1 j}$ were commercially available, whereas $\mathbf{1 b}$ and $\mathbf{1 h}$ were prepared according to standard procedures.

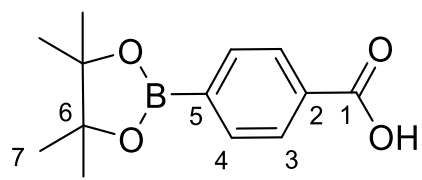

Compound 1b. ${ }^{1}$ 4-carboxyphenylboronic acid (500 mg, $3.01 \mathrm{mmol}$ ) and pinacol ( $356 \mathrm{mg}, 3.01 \mathrm{mmol}$ ) were dissolved in a mixture of THF $(10 \mathrm{~mL})$ and toluene $(10 \mathrm{~mL})$ and stirred for $2 \mathrm{~h}$ at $45^{\circ} \mathrm{C}$. Then the solvents were evaporated under reduced pressure to yield $\mathbf{1 b}$ as a white solid (740 mg, 99\%). ${ }^{1} \mathbf{H}$ NMR $\left(500 \mathrm{MHz}, \mathrm{CDCl}_{3}\right) \delta 8.10\left(\mathrm{~d}, J=8.1 \mathrm{~Hz}, 2 \mathrm{H}, \mathrm{H}_{4}\right), 7.91\left(\mathrm{~d}, J=8.1 \mathrm{~Hz}, 2 \mathrm{H}, \mathrm{H}_{3}\right), 1.36\left(\mathrm{~s}, 12 \mathrm{H}, \mathrm{H}_{7}\right) .{ }^{13} \mathrm{C} \mathrm{NMR}$ $\left(126 \mathrm{MHz}, \mathrm{CDCl}_{3}\right) \delta 172.0\left(\mathrm{C}_{1}\right), 134.9\left(2 \mathrm{C}_{3}\right), 131.5\left(\mathrm{C}_{2}\right), 129.3\left(2 \mathrm{C}_{4}\right), 84.4\left(2 \mathrm{C}_{6}\right), 25.0\left(4 \mathrm{C}_{7}\right)\left({ }^{*} \mathrm{C}_{5}\right.$ not visible in the ${ }^{13} \mathrm{C}$ NMR spectrum).

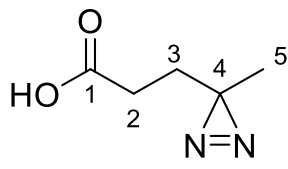

Compound $1 \mathrm{~h}^{2}{ }^{2}$ A solution of $\mathrm{NH}_{3} 7 \mathrm{M}$ in $\mathrm{MeOH}(4,5 \mathrm{~mL}, 31.3 \mathrm{mmol})$ was added at $0^{\circ} \mathrm{C}$ to a solution of levulinic acid $(400 \mu \mathrm{L}, 3.91 \mathrm{mmol})$ in $\mathrm{MeOH}(1 \mathrm{~mL})$. The reaction mixture was stirred for $3 \mathrm{~h}$ at $0^{\circ} \mathrm{C}$ and then a solution of hydroxylamine-O-sulfonic acid $(486 \mathrm{mg}, 4.30 \mathrm{mmol})$ in $\mathrm{MeOH}(2 \mathrm{~mL})$ was added at $0^{\circ} \mathrm{C}$. The reaction mixture was stirred at room temperature overnight, then $\mathrm{NH}_{3}$ was removed with air stream for $1 \mathrm{~h}$. The resulting suspension was then filtered and the filtrate concentrated under reduced pressure. The residue was taken up in $\mathrm{MeOH}(2 \mathrm{~mL})$ and cooled to $0^{\circ} \mathrm{C}$. $\mathrm{NEt}_{3}(820 \mu \mathrm{L}, 5.86 \mathrm{mmol})$ was added and the resulting mixture was stirred at $0^{\circ} \mathrm{C}$ for $5 \mathrm{~min}$ and then $\mathrm{I}_{2}$ was added in portions. The addition was continued until the colour of iodine persisted $(892 \mathrm{mg}, 3.52 \mathrm{mmol})$. The reaction mixture was stirred for $5 \mathrm{~min}$ at room temperature and diluted with ethyl acetate. The organic layer was then washed with $1 \mathrm{M} \mathrm{HCl}, 10 \% \mathrm{Na}_{2} \mathrm{~S}_{2} \mathrm{O}_{5}$ and brine, dried over $\mathrm{MgSO}_{4}$ and concentrated under vacuum to afford compound $1 \mathrm{~h}$ as a yellow oil $(288 \mathrm{mg}, 58 \%) .{ }^{1} \mathrm{H}$ NMR $\left(500 \mathrm{MHz}, \mathrm{CDCl}_{3}\right) \delta 2.20(\mathrm{t}, J=7.7 \mathrm{~Hz}, 2 \mathrm{H}$,

\footnotetext{
${ }^{1}$ Kumar, A.; Ye, G.; Ahmadibeni, Y.; Parang, K. J. Org. Chem. 2006, 71, 7915-7918.

${ }^{2}$ Ikeda, Y.; Behrman, E. J. Synth. Commun. 2008, 38, 2276-2284.
} 
$\left.\mathrm{H}_{2}\right), 1.68\left(\mathrm{t}, J=7.7 \mathrm{~Hz}, 2 \mathrm{H}, \mathrm{H}_{3}\right), 1.00\left(\mathrm{~s}, 3 \mathrm{H}, \mathrm{H}_{5}\right) .{ }^{13} \mathrm{C} \mathrm{NMR}\left(126 \mathrm{MHz}, \mathrm{CDCl}_{3}\right) \delta 178.9\left(\mathrm{C}_{1}\right), 29.4\left(\mathrm{C}_{3}\right), 28.6$ $\left(C_{2}\right), 25.1\left(C_{4}\right), 19.7\left(C_{5}\right)$.

\section{Synthesis of phosphines}

General procedure for preparation of phosphines. To a solution of (2hydroxyphenyl)diphenylphosphine (1 equiv) in DCM were successively added DCC ( 3 equiv), DMAP (4 equiv) and a carboxylic acid 1 (3 equiv). The mixture was stirred for $16 \mathrm{~h}$ at room temperature then filtered and concentrated under reduced pressure. The crude product was purified by flash chromatography to afford product $\mathbf{2}$.<smiles>O=C(Oc1ccccc1P(c1ccccc1)c1ccccc1)c1ccccc1</smiles>

Compound 2a. ${ }^{3}$ Following the general procedure for the preparation of phosphines, starting from (2hydroxyphenyl)diphenylphosphine (200 mg, $0.72 \mathrm{mmol}$ ) and benzoic acid 1a (263 mg, $2.16 \mathrm{mmol}$ ) in DCM (7 mL) and using cyclohexane/ethyl acetate (95/5) as eluent for flash chromatography purification, compound $2 \mathrm{a}$ was obtained as a white solid (247 mg, 90\%). ${ }^{1} \mathrm{H} \mathbf{N M R}\left(500 \mathrm{MHz}, \mathrm{CDCl}_{3}\right) \delta$ $7.89\left(\mathrm{~d}, J=7.4 \mathrm{~Hz}, 2 \mathrm{H}, \mathrm{H}_{3}\right), 7.55\left(\mathrm{t}, J=7.4 \mathrm{~Hz}, 1 \mathrm{H}, \mathrm{H}_{5}\right), 7.47-7.43\left(\mathrm{~m}, 1 \mathrm{H}, \mathrm{H}_{\mathrm{B}}\right), 7.39-7.33\left(\mathrm{~m}, 13 \mathrm{H}, \mathrm{H}_{4, \mathrm{~A}, \mathrm{~B}}\right)$, $7.20\left(\mathrm{t}, J=7.5 \mathrm{~Hz}, 1 \mathrm{H}, \mathrm{H}_{\mathrm{B}}\right), 6.91$ (ddd, $\left.J=7.5,4.2,1.4 \mathrm{~Hz}, 1 \mathrm{H}, \mathrm{H}_{\mathrm{B}}\right) .{ }^{13} \mathrm{C} \mathrm{NMR}\left(126 \mathrm{MHz}, \mathrm{CDCl}_{3}\right) \delta 164.3$ $\left(C_{1}\right), 152.8\left(\mathrm{Cq}_{\mathrm{B}}\right), 135.5\left(2 \mathrm{Cq}_{\mathrm{A}}\right), 134.2\left(2 \mathrm{C}_{\mathrm{A}}\right), 134.1\left(2 \mathrm{C}_{\mathrm{A}}\right), 133.6\left(\mathrm{C}_{\mathrm{B}}\right), 133.4\left(\mathrm{C}_{5}\right), 130.8\left(\mathrm{Cq}_{\mathrm{B}}\right), 130.2\left(2 \mathrm{C}_{3}\right)$, $130.0\left(C_{B}\right), 129.2\left(C_{2}\right), 129.1\left(2 C_{A}\right), 128.6\left(4 C_{A}\right), 128.3\left(2 C_{4}\right), 126.2\left(C_{B}\right), 122.7\left(C_{B}\right) .{ }^{31}$ P NMR $(202 \mathrm{MHz}$, $\left.\mathrm{CDCl}_{3}\right) \delta$-15.4. HRMS calculated for $\mathrm{C}_{25} \mathrm{H}_{20} \mathrm{O}_{2} \mathrm{P}[\mathrm{M}+\mathrm{H}]^{+}: 383.1201$; found: 383.1198.

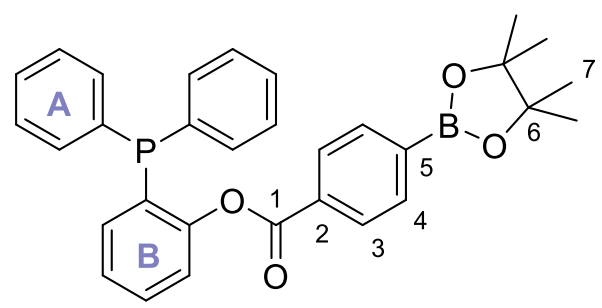

${ }^{3}$ Zhang, J.; Wang, H.; Xian, M. Org. Lett. 2009, 11, 477-480. 
Compound $\mathbf{2 b}$. Following the general procedure for the preparation of phosphines, starting from (2hydroxyphenyl)diphenylphosphine $(200 \mathrm{mg}, 0.72 \mathrm{mmol}$ ) and 4-carboxyphenylboronic acid pinacol ester $1 \mathbf{b}(535 \mathrm{mg}, 2.16 \mathrm{mmol})$ in DCM (7 mL) and using cyclohexane/ethyl acetate (96/4) as eluent for flash chromatography purification, compound $\mathbf{2} \mathbf{b}$ was obtained as a white foam ( $271 \mathrm{mg}, 74 \%$ ). ${ }^{1} \mathbf{H}$ NMR $\left(500 \mathrm{MHz}, \mathrm{CDCl}_{3}\right) \delta 7.87-7.83\left(\mathrm{~m}, 4 \mathrm{H}, \mathrm{H}_{3,4}\right), 7.46-7.42\left(\mathrm{~m}, 1 \mathrm{H}, \mathrm{H}_{\mathrm{B}}\right), 7.39-7.33\left(\mathrm{~m}, 11 \mathrm{H}, \mathrm{H}_{\mathrm{A}, \mathrm{B}}\right)$, $7.19\left(\mathrm{t}, J=7.5 \mathrm{~Hz}, 1 \mathrm{H}, \mathrm{H}_{\mathrm{B}}\right), 6.91\left(\mathrm{ddd}, J=7.5,4.2,1.4 \mathrm{~Hz}, 1 \mathrm{H}, \mathrm{H}_{\mathrm{B}}\right), 1.39\left(\mathrm{~s}, 12 \mathrm{H}, \mathrm{H}_{7}\right) .{ }^{13} \mathrm{C} \mathrm{NMR}(126 \mathrm{MHz}$, $\left.\mathrm{CDCl}_{3}\right) \delta 164.3\left(\mathrm{C}_{1}\right), 152.8\left(\mathrm{Cq}_{\mathrm{B}}\right), 135.5\left(2 \mathrm{Cq}_{\mathrm{A}}\right), 134.6\left(2 \mathrm{C}_{3}\right), 134.2\left(2 \mathrm{C}_{\mathrm{A}}\right), 134.1\left(2 \mathrm{C}_{\mathrm{A}}\right), 133.6\left(\mathrm{C}_{\mathrm{B}}\right), 131.4$ $\left(C_{2}\right), 130.8\left(C_{B}\right), 129.9\left(C_{B}\right), 129.2\left(2 C_{4}\right), 129.1\left(2 C_{A}\right), 128.7\left(2 C_{A}\right), 128.6\left(2 C_{A}\right), 126.2\left(C_{B}\right), 122.6\left(C_{B}\right)$, $84.3\left(2 \mathrm{C}_{6}\right), 25.0\left(4 \mathrm{C}_{7}\right)\left({ }^{*} \mathrm{C}_{5}\right.$ not visible in the ${ }^{13} \mathrm{C}$ NMR spectrum). ${ }^{31} \mathrm{P}$ NMR (202 $\left.\mathrm{MHz}, \mathrm{CDCl}_{3}\right) \delta-15.3$. HRMS calculated for $\mathrm{C}_{31} \mathrm{H}_{31} \mathrm{BO}_{4} \mathrm{P}[\mathrm{M}+\mathrm{H}]^{+}: 509.2053$; found: 509.2036 .

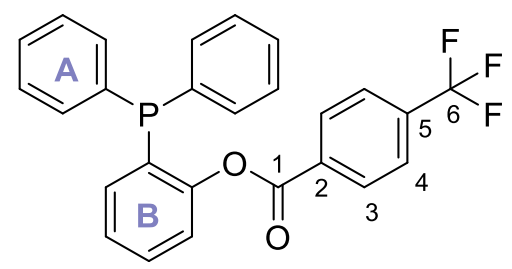

Compound 2c. Following the general procedure for the preparation of phosphines, starting from (2hydroxyphenyl)diphenylphosphine $(200 \mathrm{mg}, 0.72 \mathrm{mmol}$ ) and 4-(trifluoromethyl)benzoic acid 1c (410 $\mathrm{mg}, 2.16 \mathrm{mmol})$ in DCM $(7 \mathrm{~mL})$ and using cyclohexane/ethyl acetate $(98 / 2)$ as eluent for flash chromatography purification, compound $\mathbf{2 c}$ was obtained as a white foam ( $285 \mathrm{mg}, 88 \%$ ). ${ }^{1} \mathbf{H}$ NMR (500 $\left.\mathrm{MHz}, \mathrm{CDCl}_{3}\right) \delta 7.98\left(\mathrm{~d}, J=8.2 \mathrm{~Hz}, 2 \mathrm{H}, \mathrm{H}_{3}\right), 7.65\left(\mathrm{~d}, J=8.2 \mathrm{~Hz}, 2 \mathrm{H}, \mathrm{H}_{4}\right), 7.48-7.45\left(\mathrm{~m}, 1 \mathrm{H}, \mathrm{H}_{\mathrm{B}}\right), 7.39-7.34$ $\left(\mathrm{m}, 11 \mathrm{H}, \mathrm{H}_{\mathrm{A}}, \mathrm{B}\right), 7.23\left(\mathrm{t}, J=7.5 \mathrm{~Hz}, 1 \mathrm{H}, \mathrm{H}_{\mathrm{B}}\right), 6.92$ (ddd, $\left.J=7.5,4.3,1.4 \mathrm{~Hz}, 1 \mathrm{H}, \mathrm{H}_{\mathrm{B}}\right) .{ }^{13} \mathrm{C}$ NMR $(126 \mathrm{MHz}$, $\left.\mathrm{CDCl}_{3}\right)$ \& $163.2\left(\mathrm{C}_{1}\right), 152.5\left(\mathrm{Cq}_{\mathrm{B}}\right), 135.2\left(2 \mathrm{Cq}_{\mathrm{A}}\right), 134.8\left(\mathrm{C}_{5}\right), 134.3\left(2 \mathrm{C}_{\mathrm{A}}\right), 134.1\left(2 \mathrm{C}_{\mathrm{A}}\right), 133.7\left(\mathrm{C}_{\mathrm{B}}\right), 132.5\left(\mathrm{C}_{2}\right)$, $130.7\left(\mathrm{Cq}_{\mathrm{B}}\right), 130.6\left(2 \mathrm{C}_{3}\right), 130.1\left(\mathrm{C}_{\mathrm{B}}\right), 129.3\left(2 \mathrm{C}_{\mathrm{A}}\right), 128.8\left(2 \mathrm{C}_{\mathrm{A}}\right), 128.7\left(2 \mathrm{C}_{\mathrm{A}}\right), 126.6\left(\mathrm{C}_{\mathrm{B}}\right), 125.4\left(2 \mathrm{C}_{4}\right), 123.7$ $\left(\mathrm{C}_{6}\right), 122.5\left(\mathrm{C}_{\mathrm{B}}\right) .{ }^{31} \mathrm{P}$ NMR $\left(202 \mathrm{MHz}, \mathrm{CDCl}_{3}\right) \delta$-15.3. HRMS calculated for $\left.\mathrm{C}_{26} \mathrm{H}_{17} \mathrm{~F}_{3} \mathrm{O}_{2} \mathrm{P}[\mathrm{M}-\mathrm{H}]::\right]^{-:}: 449.0924$ ; found: 449.0927.

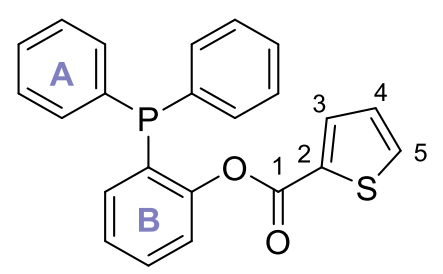

Compound 2d. Following the general procedure for the preparation of phosphines, starting from (2hydroxyphenyl)diphenylphosphine (200 mg, $0.72 \mathrm{mmol}$ ) and 2-thiophenecarboxylic acid $1 \mathbf{d}$ (276 mg, $2.16 \mathrm{mmol})$ in DCM $(7 \mathrm{~mL})$ and using cyclohexane/ethyl acetate (96/4) as eluent for flash chromatography purification, compound $\mathbf{2} \mathbf{d}$ was obtained as a white foam ( $277 \mathrm{mg}, 99 \%) .{ }^{1} \mathbf{H}$ NMR 
$\left(500 \mathrm{MHz}, \mathrm{CDCl}_{3}\right) \delta 7.67\left(\mathrm{dd}, J=3.8,1.2 \mathrm{~Hz}, 1 \mathrm{H}, \mathrm{H}_{3}\right), 7.55$ (dd, $\left.J=5.0,1.2 \mathrm{~Hz}, 1 \mathrm{H}, \mathrm{H}_{5}\right), 7.45(\mathrm{td}, J=8.1$, $\left.1.5 \mathrm{~Hz}, 1 \mathrm{H}, \mathrm{H}_{\mathrm{B}}\right), 7.41-7.33\left(\mathrm{~m}, 11 \mathrm{H}, \mathrm{H}_{\mathrm{A}, \mathrm{B}}\right), 7.20\left(\mathrm{t}, J=7.5 \mathrm{~Hz}, 1 \mathrm{H}, \mathrm{H}_{\mathrm{B}}\right), 7.05\left(\mathrm{dd}, J=4.9,3.8 \mathrm{~Hz}, 1 \mathrm{H}, \mathrm{H}_{4}\right)$, 6.92 (ddd, $\left.J=7.6,4.2,1.6 \mathrm{~Hz}, 1 \mathrm{H}, \mathrm{H}_{\mathrm{B}}\right) .{ }^{13} \mathrm{C}$ NMR $\left(126 \mathrm{MHz}, \mathrm{CDCl}_{3}\right) \delta 159.7\left(\mathrm{C}_{1}\right), 152.5\left(\mathrm{Cq}_{\mathrm{B}}\right), 135.5\left(2 \mathrm{Cq}_{\mathrm{A}}\right)$, $134.6\left(C_{3}\right), 134.2\left(2 C_{A}\right), 134.0\left(2 C_{A}\right), 133.6\left(C_{B}\right), 133.5\left(C_{5}\right), 132.6\left(C_{2}\right), 130.8\left(C_{B}\right), 129.9\left(C_{B}\right), 129.0\left(2 C_{A}\right)$, $128.6\left(2 \mathrm{C}_{\mathrm{A}}\right), 128.5\left(2 \mathrm{C}_{\mathrm{A}}\right), 127.7\left(\mathrm{C}_{4}\right), 126.3\left(\mathrm{C}_{\mathrm{B}}\right), 122.6\left(\mathrm{C}_{\mathrm{B}}\right) .{ }^{31} \mathrm{P}$ NMR $\left(202 \mathrm{MHz}, \mathrm{CDCl}_{3}\right) \delta$-15.5. HRMS calculated for $\mathrm{C}_{23} \mathrm{H}_{18} \mathrm{O}_{2} \mathrm{PS}[\mathrm{M}+\mathrm{H}]^{+}: 389.0765$; found: 389.0749 .

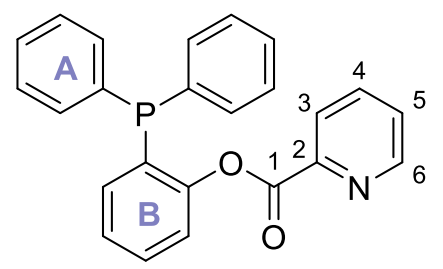

Compound 2e. Following the general procedure for the preparation of phosphines, starting from (2hydroxyphenyl)diphenylphosphine (300 mg, $1.08 \mathrm{mmol}$ ) and 2-picolinic acid 1e (398 mg, $3.23 \mathrm{mmol}$ ) in DCM (10 mL) and using cyclohexane/ethyl acetate (8/2) as eluent for flash chromatography purification, compound $2 \mathrm{e}$ was obtained as a beige foam (370 mg, 90\%). ${ }^{1} \mathbf{H} \mathbf{N M R}\left(500 \mathrm{MHz}, \mathrm{CDCl}_{3}\right) \delta$ $8.61\left(\mathrm{~d}, J=4.7 \mathrm{~Hz}, 1 \mathrm{H}, \mathrm{H}_{6}\right), 7.70\left(\mathrm{~d}, J=7.8 \mathrm{~Hz}, 1 \mathrm{H}, \mathrm{H}_{3}\right), 7.57$ (td, $\left.J=7.7,1.2 \mathrm{~Hz}, 1 \mathrm{H}, \mathrm{H}_{4}\right), 7.32-7.27(\mathrm{~m}$, $\left.2 \mathrm{H}, \mathrm{H}_{5, \mathrm{~B}}\right), 7.24-7.15\left(\mathrm{~m}, 11 \mathrm{H}, \mathrm{H}_{\mathrm{A}, \mathrm{B}}\right), 7.05\left(\mathrm{t}, J=7.5 \mathrm{~Hz}, 1 \mathrm{H}, \mathrm{H}_{\mathrm{B}}\right), 6.80$ (ddd, J = 7.5, 4.2, $1.3 \mathrm{~Hz}, 1 \mathrm{H}, \mathrm{H}_{\mathrm{B}}$ ). ${ }^{13} \mathrm{C}$ NMR $\left(126 \mathrm{MHz}, \mathrm{CDCl}_{3}\right) \delta 162.5\left(\mathrm{C}_{1}\right), 152.9\left(\mathrm{Cq}_{\mathrm{B}}\right), 150.0\left(\mathrm{C}_{6}\right), 147.0\left(\mathrm{C}_{2}\right), 136.7\left(\mathrm{C}_{4}\right), 135.4\left(2 \mathrm{Cq}_{\mathrm{A}}\right)$, $134.0\left(2 C_{A}\right), 133.9\left(2 C_{A}\right), 133.8\left(C_{B}\right), 130.5\left(C_{B}\right), 130.1\left(C_{B}\right), 129.0\left(2 C_{A}\right), 128.5\left(4 C_{A}\right), 127.0\left(C_{5}\right), 126.4$ $\left(C_{B}\right), 125.6\left(C_{3}\right), 122.5\left(C_{B}\right) .{ }^{31} \mathrm{P}$ NMR $\left(202 \mathrm{MHz}, \mathrm{CDCl}_{3}\right) \delta$-16.1. HRMS calculated for $\mathrm{C}_{24} \mathrm{H}_{19} \mathrm{NO}_{2} \mathrm{P}[\mathrm{M}+\mathrm{H}]^{+}$: 384.1153 ; found: 384.1137.

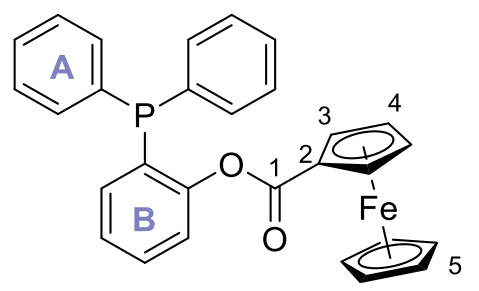

Compound 2f. Following the general procedure for the preparation of phosphines, starting from (2hydroxyphenyl)diphenylphosphine ( $200 \mathrm{mg}, 0.72 \mathrm{mmol}$ ) and ferrocenecarboxylic acid $1 \mathbf{1 f}$ ( $496 \mathrm{mg}, 2.16$ $\mathrm{mmol}$ ) in DCM (7 mL) and using cyclohexane/ethyl acetate (96/4) as eluent for flash chromatography purification, compound $\mathbf{2 f}$ was obtained as an orange foam (333 mg, 95\%). ${ }^{1} \mathbf{H} \mathbf{N M R}\left(500 \mathrm{MHz}, \mathrm{CDCl}_{3}\right.$ ) $\delta 7.48-7.40\left(\mathrm{~m}, 12 \mathrm{H}, \mathrm{H}_{\mathrm{A}, \mathrm{B}}\right), 7.19\left(\mathrm{t}, J=7.3 \mathrm{~Hz}, 1 \mathrm{H}, \mathrm{H}_{\mathrm{B}}\right), 6.90$ (ddd, $\left.J=7.6,4.0,1.3 \mathrm{~Hz}, 1 \mathrm{H}, \mathrm{H}_{\mathrm{B}}\right), 4.74-$ $4.73\left(\mathrm{~m}, 2 \mathrm{H}, \mathrm{H}_{3}\right), 4.44-4.43\left(\mathrm{~m}, 2 \mathrm{H}, \mathrm{H}_{4}\right), 4.33\left(\mathrm{~s}, 5 \mathrm{H}, \mathrm{H}_{5}\right) .{ }^{13} \mathrm{C} \mathrm{NMR}\left(126 \mathrm{MHz}, \mathrm{CDCl}_{3}\right) \delta 169.7\left(\mathrm{C}_{1}\right), 153.1$ 
$\left(\mathrm{Cq}_{\mathrm{B}}\right), 136.0\left(2 \mathrm{Cq}_{\mathrm{A}}\right), 134.0\left(2 \mathrm{C}_{\mathrm{A}}\right), 133.8\left(2 \mathrm{C}_{\mathrm{A}}\right), 133.6\left(\mathrm{C}_{\mathrm{B}}\right), 129.9\left(\mathrm{C}_{\mathrm{B}}\right), 129.7\left(\mathrm{Cq}_{\mathrm{B}}\right), 128.9\left(2 \mathrm{C}_{\mathrm{A}}\right), 128.6\left(2 \mathrm{C}_{\mathrm{A}}\right)$, $128.5\left(2 C_{A}\right), 125.8\left(C_{B}\right), 122.2\left(C_{B}\right), 71.7\left(2 C_{4}\right), 70.5\left(2 C_{3}\right), 70.1\left(5 C_{5}\right), 70.0\left(C_{2}\right) .{ }^{31} \mathrm{P} \mathrm{NMR}\left(202 \mathrm{MHz}, \mathrm{CDCl}_{3}\right)$ $\delta$-16.0. HRMS calculated for $\mathrm{C}_{29} \mathrm{H}_{24} \mathrm{FeO}_{2} \mathrm{P}[\mathrm{M}+\mathrm{H}]^{+}: 491.0863$; found: 491.0846.<smiles>CC(=O)Oc1ccccc1P(c1ccccc1)c1ccccc1</smiles>

Compound $\mathbf{2 g} .{ }^{4}$ Following the general procedure for the preparation of phosphines, starting from (2hydroxyphenyl)diphenylphosphine ( $200 \mathrm{mg}, 0.72 \mathrm{mmol}$ ) and acetic acid $1 \mathrm{~g}(123 \mu \mathrm{L}, 2.16 \mathrm{mmol})$ in DCM $(7 \mathrm{~mL})$ and using cyclohexane/ethyl acetate $(95 / 5)$ as eluent for flash chromatography purification, compound $\mathbf{2 g}$ was obtained as a white foam (219 mg, 95\%). ${ }^{1} \mathbf{H}$ NMR $\left(500 \mathrm{MHz}, \mathrm{CDCl}_{3}\right) \delta 7.42-7.39$ $\left(m, 11 \mathrm{H}, \mathrm{H}_{\mathrm{A}}, \mathrm{B}\right), 7.21-7.15\left(\mathrm{~m}, 2 \mathrm{H}, \mathrm{H}_{\mathrm{B}}\right), 6.93-6.91\left(\mathrm{~m}, 1 \mathrm{H}, \mathrm{H}_{\mathrm{B}}\right), 2.02\left(\mathrm{~s}, 3 \mathrm{H}, \mathrm{H}_{2}\right) .{ }^{13} \mathrm{C}$ NMR $(126 \mathrm{MHz}$, $\left.\mathrm{CDCl}_{3}\right) \delta 168.9\left(\mathrm{C}_{1}\right), 152.7\left(\mathrm{Cq}_{\mathrm{B}}\right), 135.5\left(2 \mathrm{Cq}_{\mathrm{A}}\right), 134.0\left(2 \mathrm{C}_{\mathrm{A}}\right), 133.9\left(2 \mathrm{C}_{\mathrm{A}}\right), 133.7\left(\mathrm{C}_{\mathrm{B}}\right), 130.4\left(\mathrm{Cq}_{\mathrm{B}}\right), 129.9$ $\left(C_{B}\right), 129.1\left(2 C_{A}\right), 128.6\left(4 C_{A}\right), 126.2\left(C_{B}\right), 122.6\left(C_{B}\right), 20.6\left(C_{2}\right) .{ }^{31}$ P NMR $\left(202 \mathrm{MHz}, C D C l_{3}\right) \delta$-15.8. HRMS calculated for $\mathrm{C}_{20} \mathrm{H}_{18} \mathrm{O}_{2} \mathrm{P}[\mathrm{M}+\mathrm{H}]^{+}: 321.1044$; found: 321.1038 .

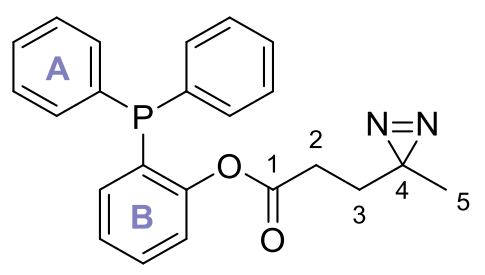

Compound $\mathbf{2 h} .^{5}$ Following the general procedure for the preparation of phosphines, starting from (2hydroxyphenyl)diphenylphosphine (208 $\mathrm{mg}, 0.75 \mathrm{mmol}$ ) and 3-methyl-diazirine-3-propanoic acid $\mathbf{1 h}$ (288 mg, $2.24 \mathrm{mmol})$ in DCM (7 mL) and using cyclohexane/ethyl acetate (96/4) as eluent for flash chromatography purification, compound $\mathbf{2 h}$ was obtained as a colorless oil ( $242 \mathrm{mg}, 83 \%$ ). ${ }^{1} \mathbf{H}$ NMR $\left(500 \mathrm{MHz}, \mathrm{CDCl}_{3}\right) \delta 7.41-7.33\left(\mathrm{~m}, 11 \mathrm{H}, \mathrm{H}_{\mathrm{A}, \mathrm{B}}\right), 7.21-7.19\left(\mathrm{~m}, 1 \mathrm{H}, \mathrm{H}_{\mathrm{B}}\right), 7.15\left(\mathrm{t}, J=7.5 \mathrm{~Hz}, 1 \mathrm{H}, \mathrm{H}_{\mathrm{B}}\right), 6.86$ (ddd, J = 7.6, 4.4, $\left.1.5 \mathrm{~Hz}, 1 \mathrm{H}, \mathrm{H}_{\mathrm{B}}\right), 2.15\left(\mathrm{t}, J=7.9 \mathrm{~Hz}, 2 \mathrm{H}, \mathrm{H}_{2}\right), 1.56\left(\mathrm{t}, J=7.9 \mathrm{~Hz}, 2 \mathrm{H}, \mathrm{H}_{3}\right), 0.99\left(\mathrm{~s}, 3 \mathrm{H}, \mathrm{H}_{5}\right)$. ${ }^{13} \mathrm{C}$ NMR $\left(126 \mathrm{MHz}, \mathrm{CDCl}_{3}\right) \delta 170.1\left(\mathrm{C}_{1}\right), 152.6\left(\mathrm{Cq}_{\mathrm{B}}\right), 135.5\left(2 \mathrm{Cq}_{\mathrm{A}}\right), 134.1\left(2 \mathrm{C}_{\mathrm{A}}\right), 133.9\left(2 \mathrm{C}_{\mathrm{A}}\right), 133.7\left(\mathrm{C}_{\mathrm{B}}\right)$, $130.3\left(\mathrm{Cq}_{\mathrm{B}}\right), 130.0\left(\mathrm{C}_{\mathrm{B}}\right), 129.1\left(2 \mathrm{C}_{\mathrm{A}}\right), 128.7\left(4 \mathrm{C}_{\mathrm{A}}\right), 126.3\left(\mathrm{C}_{\mathrm{B}}\right), 122.6\left(\mathrm{C}_{\mathrm{B}}\right), 29.4\left(\mathrm{C}_{3}\right), 28.6\left(\mathrm{C}_{2}\right), 25.0\left(\mathrm{C}_{4}\right)$,

\footnotetext{
${ }^{4}$ Kitoun, C.; Fonvielle, M.; Sakkas, N.; Lefresne, M.; Djago, F.; Blancart Remaury, Q.; Poinot, P.; Arthur, M.; Etheve-Quelquejeu, M.; Iannazzo, L. Org. Lett. 2020, 22, 8034-8038.

${ }^{5}$ Ahad, A. M.; Jensen, S. M.; Jewett, J. C. Org. Lett. 2013, 15, 5060-5063.
} 
19.6 $\left(\mathrm{C}_{5}\right) .{ }^{31} \mathrm{P}$ NMR $\left(202 \mathrm{MHz}, \mathrm{CDCl}_{3}\right) \delta$-15.8. HRMS calculated for $\mathrm{C}_{23} \mathrm{H}_{22} \mathrm{~N}_{2} \mathrm{O}_{2} \mathrm{P}[\mathrm{M}+\mathrm{H}]^{+}: 389.1419$; found: 389.1417.

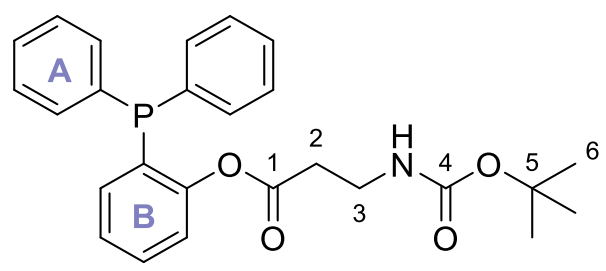

Compound 2i. ${ }^{6}$ Following the general procedure for the preparation of phosphines, starting from (2hydroxyphenyl)diphenylphosphine (200 mg, $0.72 \mathrm{mmol}$ ) and Boc- $\beta$-Ala-OH 1i (408 mg, $2.16 \mathrm{mmol}$ ) in DCM $(7 \mathrm{~mL})$ and using cyclohexane/ethyl acetate $(8 / 2)$ as eluent for flash chromatography purification, compound $2 \mathbf{i}$ was obtained as a white foam (321 mg, 99\%). ${ }^{1} \mathbf{H}$ NMR $\left(500 \mathrm{MHz}, \mathrm{CDCl}_{3}\right) \delta 7.39-7.34(\mathrm{~m}$, $\left.11 \mathrm{H}, \mathrm{H}_{\mathrm{A}}, \mathrm{B}\right), 7.17-7.12\left(\mathrm{~m}, 2 \mathrm{H}, \mathrm{H}_{\mathrm{B}}\right), 6.89-6.87\left(\mathrm{~m}, 1 \mathrm{H}, \mathrm{H}_{\mathrm{B}}\right), 5.15(\mathrm{bs}, 1 \mathrm{H}, \mathrm{NH}), 3.33-3.32\left(\mathrm{~m}, 2 \mathrm{H}, \mathrm{H}_{3}\right)$, $2.51\left(\mathrm{t}, J=5.4 \mathrm{~Hz}, 2 \mathrm{H}, \mathrm{H}_{2}\right), 1.47\left(\mathrm{~s}, 9 \mathrm{H}, \mathrm{H}_{6}\right) .{ }^{13} \mathrm{C} \mathrm{NMR}\left(126 \mathrm{MHz}, \mathrm{CDCl}_{3}\right) \delta 170.3\left(\mathrm{C}_{1}\right), 155.7\left(\mathrm{C}_{4}\right), 152.6$ $\left(\mathrm{Cq}_{\mathrm{B}}\right), 135.3\left(2 \mathrm{Cq}_{\mathrm{A}}\right), 133.9\left(2 \mathrm{C}_{\mathrm{A}}\right), 133.8\left(3 \mathrm{C}, 2 \mathrm{C}_{\mathrm{A}}\right.$ and $\left.\mathrm{C}_{\mathrm{B}}\right), 130.1\left(\mathrm{Cq}_{\mathrm{B}}\right), 130.0\left(\mathrm{C}_{\mathrm{B}}\right), 129.1\left(2 \mathrm{C}_{\mathrm{A}}\right), 128.6\left(4 \mathrm{C}_{\mathrm{A}}\right)$, 126.3 $\left(C_{B}\right), 122.5\left(C_{B}\right), 79.1\left(C_{5}\right), 35.9\left(C_{3}\right), 34.7\left(C_{2}\right), 28.4\left(3 C_{6}\right) .{ }^{31}$ P NMR $\left(202 \mathrm{MHz}, \mathrm{CDCl}_{3}\right)$ 8-16.7. HRMS calculated for $\mathrm{C}_{26} \mathrm{H}_{29} \mathrm{NO}_{4} \mathrm{P}[\mathrm{M}+\mathrm{H}]^{+}: 450.1834$; found: 450.1824.

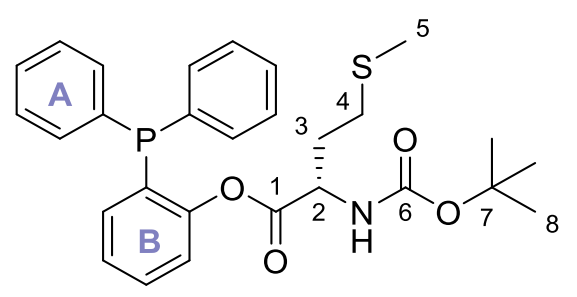

Compound 2j. Following the general procedure for the preparation of phosphines, starting from (2hydroxyphenyl)diphenylphosphine (200 mg, $0.72 \mathrm{mmol}$ ) and Boc-Met-OH 1j (538 mg, $2.16 \mathrm{mmol}$ ) in $\operatorname{DCM}(7 \mathrm{~mL})$ and using cyclohexane/ethyl acetate (9/1) as eluent for flash chromatography purification, compound $2 \mathbf{j}$ was obtained as a white foam (335 mg, 91\%). ${ }^{1} \mathbf{H}$ NMR $\left(500 \mathrm{MHz}, \mathrm{CDCl}_{3}\right) \delta 7.40-7.28(\mathrm{~m}$, $\left.11 \mathrm{H}, \mathrm{H}_{\mathrm{A}} \mathrm{B}\right), 7.19$ (dd, $J=7.7,4.0 \mathrm{~Hz}, 1 \mathrm{H}, \mathrm{H}_{\mathrm{B}}$ ), $7.14\left(\mathrm{t}, J=7.5 \mathrm{~Hz}, 1 \mathrm{H}, \mathrm{H}_{\mathrm{B}}\right.$ ), 6.82 (ddd, J = 7.6, 4.1, $1.5 \mathrm{~Hz}$, $\left.1 \mathrm{H}, \mathrm{H}_{\mathrm{B}}\right), 5.01(\mathrm{bd}, J=7.3 \mathrm{~Hz}, 1 \mathrm{H}, \mathrm{NH}), 4.48-4.43\left(\mathrm{~m}, 1 \mathrm{H}, \mathrm{H}_{2}\right), 2.56-2.46\left(\mathrm{~m}, 2 \mathrm{H}, \mathrm{H}_{4}\right), 2.15-2.08(\mathrm{~m}$, $\left.1 \mathrm{H}, \mathrm{H}_{3}\right), 2.04\left(\mathrm{~s}, 3 \mathrm{H}, \mathrm{H}_{5}\right), 1.82-1.75\left(\mathrm{~m}, 1 \mathrm{H}, \mathrm{H}_{3}\right), 1.46\left(\mathrm{~s}, 9 \mathrm{H}, \mathrm{H}_{8}\right) .{ }^{13} \mathrm{C} \mathrm{NMR}\left(126 \mathrm{MHz}, \mathrm{CDCl}_{3}\right) \delta 170.1\left(\mathrm{C}_{1}\right)$, $155.2\left(C_{6}\right), 152.5\left(\mathrm{Cq}_{B}\right), 135.2\left(2 \mathrm{Cq}_{\mathrm{A}}\right), 133.9\left(2 \mathrm{C}_{\mathrm{A}}\right), 133.7\left(3 \mathrm{C}, 2 \mathrm{C}_{\mathrm{A}}\right.$ and $\left.\mathrm{C}_{\mathrm{B}}\right), 130.0\left(\mathrm{C}_{\mathrm{B}}\right), 129.8\left(\mathrm{Cq}_{\mathrm{B}}\right), 129.0$ $\left(2 C_{A}\right), 128.6\left(4 C_{A}\right), 126.4\left(C_{B}\right), 122.2\left(C_{B}\right), 79.8\left(C_{7}\right), 52.9\left(C_{2}\right), 31.4\left(C_{3}\right), 29.9\left(C_{4}\right), 28.3\left(3 C_{8}\right), 15.3\left(C_{5}\right)$.

${ }^{6}$ Jiang, J.; Taniguchia, M.; Lindsey, J. S. NewJ. Chem. 2015, 39, 4534-4550. 
${ }^{31}$ P NMR (202 MHz, $\left.\mathrm{CDCl}_{3}\right) \delta$-17.1. HRMS calculated for $\mathrm{C}_{28} \mathrm{H}_{33} \mathrm{NO}_{4} \mathrm{PS}[\mathrm{M}+\mathrm{H}]^{+}: 510.1868$; found: 510.1852. [ $\alpha]_{\mathrm{D}}:-44.2^{\circ}(9,0 \mathrm{mg} / \mathrm{mL}, \mathrm{MeOH})$.

\section{Traceless Staudinger ligation}

General procedure for the traceless Staudinger ligation. A solution of azido 3 (1 equiv) and a phosphine phenyl ester 2 ( 1 equiv) in a mixture of THF and water (10/1) was stirred at $40^{\circ} \mathrm{C}$ for $16 \mathrm{~h}$. Then, the mixture was diluted with DCM and washed with brine, dried over $\mathrm{MgSO}_{4}$, filtered, and concentrated under reduced pressure. The crude product was purified by flash chromatography to afford compound 4.

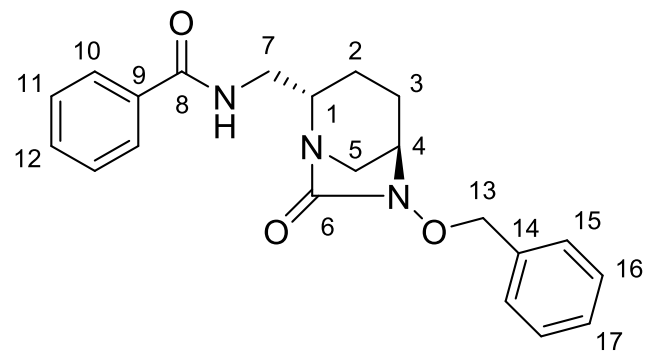

Compound 4a. Following the general procedure for the traceless Staudinger ligation, compound $4 \mathbf{a}$ was obtained as a colourless oil ( $83 \mathrm{mg}, 81 \%)$ after purification by flash chromatography using cyclohexane/ethyl acetate (3/7) as eluent, starting from azido $3(81 \mathrm{mg}, 0.28 \mathrm{mmol})$ and ester phenylphosphine 2a (108 mg, $0.28 \mathrm{mmol})$ in THF/ $\mathrm{H}_{2} \mathrm{O}(4,4 \mathrm{~mL}) .{ }^{1} \mathrm{H}$ NMR $\left(500 \mathrm{MHz}, \mathrm{CDCl}_{3}\right) \delta 7.76(\mathrm{~d}, \mathrm{~J}=$ $7.5 \mathrm{~Hz}, 2 \mathrm{H}, \mathrm{H}_{10}$ ), $7.45-7.33\left(\mathrm{~m}, 8 \mathrm{H}, \mathrm{H}_{11,12,15,16,17)} 6.90\right.$ (bs, $1 \mathrm{H}, \mathrm{NH}$ ), 5.01 (d, J=11.5 Hz, 1H, $\mathrm{H}_{13}$ ), 4.88 $\left(d, J=11.5 \mathrm{~Hz}, 1 \mathrm{H}, \mathrm{H}_{13}\right), 3.67-3.60\left(\mathrm{~m}, 3 \mathrm{H}, \mathrm{H}_{1,7}\right), 3.34-3.33\left(\mathrm{~m}, 1 \mathrm{H}, \mathrm{H}_{4}\right), 3.01\left(\mathrm{~d}, J=11.8 \mathrm{~Hz}, 1 \mathrm{H}, \mathrm{H}_{5}\right)$, $2.83\left(\mathrm{bd}, J=11.7 \mathrm{~Hz}, 1 \mathrm{H}, \mathrm{H}_{5}\right), 2.06-1.97\left(\mathrm{~m}, 2 \mathrm{H}, \mathrm{H}_{2,3}\right), 1.67-1.61\left(\mathrm{~m}, 1 \mathrm{H} ; \mathrm{H}_{3}\right), 1.47-1.41\left(\mathrm{~m}, 1 \mathrm{H}, \mathrm{H}_{2}\right)$. ${ }^{13} \mathrm{C} \mathrm{NMR}\left(126 \mathrm{MHz}, \mathrm{CDCl}_{3}\right) \delta 169.4\left(\mathrm{C}_{6}\right), 167.8\left(\mathrm{C}_{8}\right), 135.9\left(\mathrm{C}_{14}\right), 134.2\left(\mathrm{C}_{9}\right), 131.7\left(\mathrm{C}_{12}\right), 129.4\left(2 \mathrm{C}_{15}\right), 128.9$ $\left(C_{17}\right), 128.7\left(4 \mathrm{C}, 2 \mathrm{C}_{11}\right.$ and $\left.2 \mathrm{C}_{16}\right), 127.2\left(2 \mathrm{C}_{10}\right), 78.4\left(\mathrm{C}_{13}\right), 58.5\left(\mathrm{C}_{4}\right), 56.4\left(\mathrm{C}_{1}\right), 43.8\left(\mathrm{C}_{5}\right), 40.8\left(\mathrm{C}_{7}\right), 20.9\left(\mathrm{C}_{2}\right)$, $20.2\left(C_{3}\right)$. HRMS calculated for $\mathrm{C}_{21} \mathrm{H}_{24} \mathrm{~N}_{3} \mathrm{O}_{3}[\mathrm{M}+\mathrm{H}]^{+}: 366.1818$; found: 366.1806 . [ $\left.\alpha\right]_{D}:-29.7^{\circ}(11$ $\mathrm{mg} / \mathrm{mL}, \mathrm{MeOH})$. 


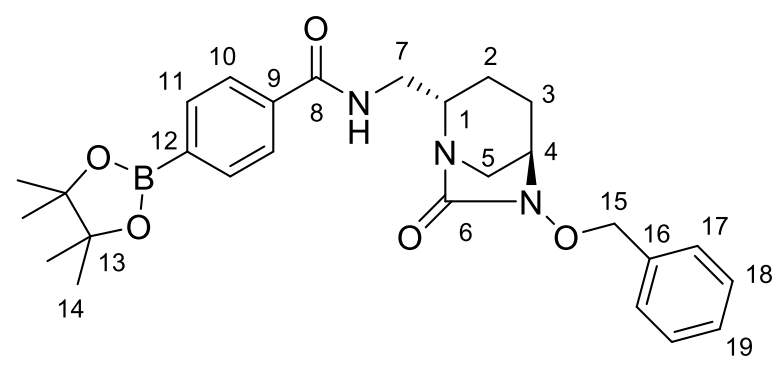

Compound $\mathbf{4 b}$. Following the general procedure for the traceless Staudinger ligation, compound $\mathbf{4 b}$ was obtained as a white foam ( $80 \mathrm{mg}, 52 \%$ ) after purification by flash chromatography using cyclohexane/ethyl acetate (3/7) as eluent, starting from azido 3 (90 mg, $0.31 \mathrm{mmol}$ ) and ester phenylphosphine $\mathbf{2 b}$ (160 mg, $0.31 \mathrm{mmol})$ in THF/ $\mathrm{H}_{2} \mathrm{O}(5,5 \mathrm{~mL}) .{ }^{1} \mathbf{H} \mathbf{N M R}\left(500 \mathrm{MHz}, \mathrm{CDCl}_{3}\right) \delta 7.81(\mathrm{~d}, J$ $\left.=8.0 \mathrm{~Hz}, 2 \mathrm{H}, \mathrm{H}_{11}\right), 7.74\left(\mathrm{~d}, J=8.1 \mathrm{~Hz}, 2 \mathrm{H}, \mathrm{H}_{10}\right), 7.41-7.39\left(\mathrm{~m}, 2 \mathrm{H}, \mathrm{H}_{17}\right), 7.37-7.31\left(\mathrm{~m}, 3 \mathrm{H}, \mathrm{H}_{18,19}\right), 6.85$ (bs, $1 \mathrm{H}, \mathrm{NH}$ ), $5.02\left(\mathrm{~d}, J=11.5 \mathrm{~Hz}, 1 \mathrm{H}, \mathrm{H}_{15}\right), 4.88\left(\mathrm{~d}, J=11.5 \mathrm{~Hz}, 1 \mathrm{H}, \mathrm{H}_{15}\right), 3.66-3.60\left(\mathrm{~m}, 3 \mathrm{H}, \mathrm{H}_{1,7}\right), 3.33$ $-3.31\left(\mathrm{~m}, 1 \mathrm{H}, \mathrm{H}_{4}\right), 2.99\left(\mathrm{~d}, J=11.8 \mathrm{~Hz}, 1 \mathrm{H}, \mathrm{H}_{5}\right), 2.84\left(\mathrm{bd}, J=11.5 \mathrm{~Hz}, 1 \mathrm{H}, \mathrm{H}_{5}\right), 2.08-1.97\left(\mathrm{~m}, 2 \mathrm{H}, \mathrm{H}_{2,3}\right)$, $1.67-1.60\left(\mathrm{~m}, 1 \mathrm{H}, \mathrm{H}_{3}\right), 1.46-1.41\left(\mathrm{~m}, 1 \mathrm{H}, \mathrm{H}_{2}\right), 1.33\left(\mathrm{~s}, 12 \mathrm{H}, \mathrm{H}_{14}\right) .{ }^{13} \mathrm{C} \mathrm{NMR}\left(126 \mathrm{MHz}, \mathrm{CDCl}_{3}\right) \delta 169.9$ $\left(C_{6}\right), 167.7\left(C_{8}\right), 136.4\left(C_{9}\right), 135.8\left(C_{16}\right), 134.9\left(2 C_{11}\right), 129.3\left(2 C_{17}\right), 128.8\left(C_{19}\right), 128.6\left(2 C_{18}\right), 126.3\left(2 C_{10}\right)$, $84.1\left(2 C_{13}\right), 78.3\left(C_{15}\right), 58.6\left(C_{4}\right), 56.3\left(C_{1}\right), 43.8\left(C_{5}\right), 40.8\left(C_{7}\right), 24.9\left(4 C_{14}\right), 20.8\left(C_{2}\right), 20.2\left(C_{3}\right) .\left({ }^{*} C_{12}\right.$ not visible in the ${ }^{13} \mathrm{C}$ NMR spectrum). HRMS calculated for $\mathrm{C}_{27} \mathrm{H}_{35} \mathrm{BN}_{3} \mathrm{O}_{5}[\mathrm{M}+\mathrm{H}]^{+}: 492.2670$; found: 492.2653. $[\alpha]_{\mathrm{D}}:-28.9^{\circ}(6,1 \mathrm{mg} / \mathrm{mL}, \mathrm{MeOH})$.

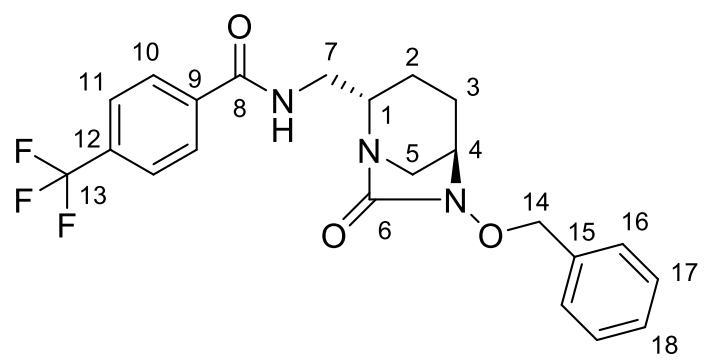

Compound 4c. Following the general procedure for the traceless Staudinger ligation, compound $\mathbf{4 c}$ was obtained as a white solid (116 mg, 77\%) after purification by flash chromatography using cyclohexane/ethyl acetate (4/6) as eluent, starting from azido 3 (100 mg, $0.35 \mathrm{mmol}$ ) and ester phenylphosphine 2c (157 mg, $0.35 \mathrm{mmol})$ in THF/ $\mathrm{H}_{2} \mathrm{O}(5,5 \mathrm{~mL}) .{ }^{1} \mathrm{H}$ NMR $\left(500 \mathrm{MHz}, \mathrm{CDCl}_{3}\right) \delta 7.85(\mathrm{~d}, J=$ $\left.8.2 \mathrm{~Hz}, 2 \mathrm{H}, \mathrm{H}_{10}\right), 7.55\left(\mathrm{~d}, \mathrm{~J}=8.2 \mathrm{~Hz}, 2 \mathrm{H}, \mathrm{H}_{11}\right), 7.39-7.36\left(\mathrm{~m}, 2 \mathrm{H}, \mathrm{H}_{16}\right), 7.35-7.29\left(\mathrm{~m}, 3 \mathrm{H}, \mathrm{H}_{17,18}\right), 7.24$ (bs, $1 \mathrm{H}, \mathrm{NH}), 4.99\left(\mathrm{~d}, J=11.5 \mathrm{~Hz}, 1 \mathrm{H}, \mathrm{H}_{14}\right), 4.87\left(\mathrm{~d}, J=11.5 \mathrm{~Hz}, 1 \mathrm{H}, \mathrm{H}_{14}\right), 3.76-3.69\left(\mathrm{~m}, 1 \mathrm{H}, \mathrm{H}_{7}\right), 3.66-$ $3.58\left(\mathrm{~m}, 2 \mathrm{H}, \mathrm{H}_{1,7}\right), 3.38-3.36\left(\mathrm{~m}, 1 \mathrm{H}, \mathrm{H}_{4}\right), 3.08\left(\mathrm{~d}, J=11.9 \mathrm{~Hz}, 1 \mathrm{H}, \mathrm{H}_{5}\right), 2.82\left(\mathrm{bd}, J=11.5 \mathrm{~Hz}, 1 \mathrm{H}, \mathrm{H}_{5}\right)$, $2.05-1.97\left(m, 2 \mathrm{H}, \mathrm{H}_{2,3}\right), 1.69-1.62\left(\mathrm{~m}, 1 \mathrm{H}, \mathrm{H}_{3}\right), 1.48-1.43\left(\mathrm{~m}, 1 \mathrm{H}, \mathrm{H}_{2}\right) .{ }^{13} \mathrm{C} \mathrm{NMR}\left(126 \mathrm{MHz}, \mathrm{CDCl}_{3}\right) \delta$ $170.1\left(C_{6}\right), 166.4\left(C_{8}\right), 137.4\left(C_{9}\right), 135.6\left(C_{15}\right), 132.9\left(C_{12}\right), 129.2\left(2 C_{16}\right), 128.8\left(C_{18}\right), 128.6\left(2 C_{17}\right), 127.7$ 
$\left(2 C_{10}\right), 125.4\left(2 C_{11}\right), 123.7\left(C_{13}\right), 78.3\left(C_{14}\right), 58.5\left(C_{4}\right), 56.6\left(C_{1}\right), 44.0\left(C_{5}\right), 40.8\left(C_{7}\right), 20.6\left(C_{2}\right), 20.1\left(C_{3}\right)$. HRMS calculated for $\mathrm{C}_{22} \mathrm{H}_{23} \mathrm{~F}_{3} \mathrm{~N}_{3} \mathrm{O}_{3}[\mathrm{M}+\mathrm{H}]^{+}: 434.1692$; found: 434.1674 . [ $\left.\alpha\right]_{\mathrm{D}}:-29.6^{\circ}(9,9 \mathrm{mg} / \mathrm{mL}$, $\mathrm{MeOH})$.

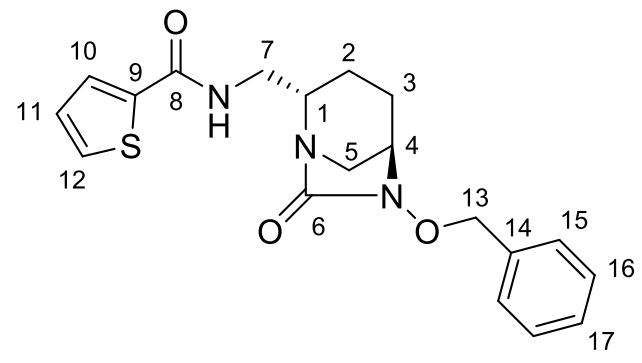

Compound $\mathbf{4 d}$. Following the general procedure for the traceless Staudinger ligation, compound $\mathbf{4 d}$ was obtained as a white foam (105 mg, 85\%) after purification by flash chromatography using cyclohexane/ethyl acetate (2/8) as eluent, starting from azido $3(95 \mathrm{mg}, 0.33 \mathrm{mmol}$ ) and ester phenylphosphine $2 \mathrm{~d}(128 \mathrm{mg}, 0.33 \mathrm{mmol})$ in THF/ $\mathrm{H}_{2} \mathrm{O}(5,5 \mathrm{~mL}) .{ }^{1} \mathrm{H} \mathbf{N M R}\left(500 \mathrm{MHz}, \mathrm{CDCl}_{3}\right) \delta 7.52(\mathrm{~d}, J$ $\left.=3.1 \mathrm{~Hz}, 1 \mathrm{H}, \mathrm{H}_{10}\right), 7.41-7.39\left(\mathrm{~m}, 3 \mathrm{H}, \mathrm{H}_{12,15}\right), 7.36-7.30\left(\mathrm{~m}, 3 \mathrm{H}, \mathrm{H}_{16,17}\right), 6.99-6.95\left(\mathrm{~m}, 2 \mathrm{H}, \mathrm{H}_{11}\right.$ and $\mathrm{NH}), 5.01\left(\mathrm{~d}, J=11.4 \mathrm{~Hz}, 1 \mathrm{H}, \mathrm{H}_{13}\right), 4.87\left(\mathrm{~d}, J=11.4 \mathrm{~Hz}, 1 \mathrm{H}, \mathrm{H}_{13}\right), 3.65-3.58\left(\mathrm{~m}, 3 \mathrm{H}, \mathrm{H}_{1,7}\right), 3.34-3.33$ $\left(\mathrm{m}, 1 \mathrm{H}, \mathrm{H}_{4}\right), 3.03\left(\mathrm{~d}, J=11.9 \mathrm{~Hz}, 1 \mathrm{H}, \mathrm{H}_{5}\right), 2.85\left(\mathrm{bd}, J=11.5 \mathrm{~Hz}, 1 \mathrm{H}, \mathrm{H}_{5}\right), 2.03-1.95\left(\mathrm{~m}, 2 \mathrm{H}, \mathrm{H}_{2,3}\right), 1.67-$ $1.60\left(\mathrm{~m}, 1 \mathrm{H}, \mathrm{H}_{3}\right), 1.47-1.42\left(\mathrm{~m}, 1 \mathrm{H}, \mathrm{H}_{2}\right) .{ }^{13} \mathrm{C} \mathrm{NMR}\left(126 \mathrm{MHz}, \mathrm{CDCl}_{3}\right) \delta 170.0\left(\mathrm{C}_{6}\right), 162.2\left(\mathrm{C}_{8}\right), 138.9\left(\mathrm{C}_{9}\right)$, $135.7\left(C_{14}\right), 130.1\left(C_{12}\right), 129.2\left(2 C_{15}\right), 128.8\left(C_{17}\right), 128.5\left(2 C_{16}\right), 128.2\left(C_{10}\right), 127.6\left(C_{11}\right), 78.2\left(C_{13}\right), 58.5$ $\left(C_{4}\right), 56.4\left(C_{1}\right), 44.0\left(C_{5}\right), 40.6\left(C_{7}\right), 20.5\left(C_{2}\right), 20.1\left(C_{3}\right)$. HRMS calculated for $\mathrm{C}_{19} \mathrm{H}_{22} \mathrm{~N}_{3} \mathrm{O}_{3} \mathrm{~S}[\mathrm{M}+\mathrm{H}]^{+}$: 372.1382 ; found: $372.1366 .[\alpha]_{\mathrm{D}}:-36.4^{\circ}(6,7 \mathrm{mg} / \mathrm{mL}, \mathrm{MeOH})$.

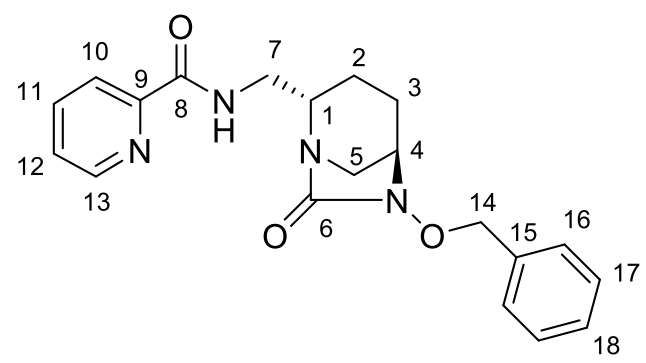

Compound 4e. Following the general procedure for the traceless Staudinger ligation, compound $\mathbf{4 e}$ was obtained as a white foam (58 $\mathrm{mg}, 51 \%$ ) after purification by flash chromatography using cyclohexane/ethyl acetate (3/7) as eluent, starting from azido $3(90 \mathrm{mg}, 0.31 \mathrm{mmol})$ and ester phenylphosphine $2 \mathrm{e}(120 \mathrm{mg}, 0.31 \mathrm{mmol})$ in THF/ $\mathrm{H}_{2} \mathrm{O}(4,4 \mathrm{~mL}) .{ }^{1} \mathrm{H}$ NMR $\left(500 \mathrm{MHz}, \mathrm{CDCl}_{3}\right) \delta 8.51$ (ddd, $\left.J=4.8,1.6,0.9 \mathrm{~Hz}, 1 \mathrm{H}, \mathrm{H}_{13}\right), 8.29(\mathrm{bs}, 1 \mathrm{H}, \mathrm{NH}), 8.13\left(\mathrm{dt}, J=7.8,1.0 \mathrm{~Hz}, 1 \mathrm{H}, \mathrm{H}_{10}\right), 7.79(\mathrm{td}, J=7.7,1.7 \mathrm{~Hz}$, 


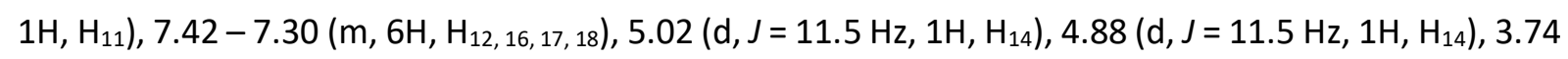
- $3.59\left(\mathrm{~m}, 3 \mathrm{H}, \mathrm{H}_{1}, 7\right), 3.33-3.32\left(\mathrm{~m}, 1 \mathrm{H}, \mathrm{H}_{4}\right), 3.03\left(\mathrm{~d}, J=11.9 \mathrm{~Hz}, 1 \mathrm{H}, \mathrm{H}_{5}\right), 2.91\left(\mathrm{bd}, J=11.8 \mathrm{~Hz}, 1 \mathrm{H}, \mathrm{H}_{5}\right)$, $2.09-1.98\left(m, 2 \mathrm{H}, \mathrm{H}_{2,3}\right), 1.68-1.60\left(\mathrm{~m}, 1 \mathrm{H}, \mathrm{H}_{3}\right), 1.48-1.42\left(\mathrm{~m}, 1 \mathrm{H}, \mathrm{H}_{2}\right) .{ }^{13} \mathrm{C}$ NMR $\left(126 \mathrm{MHz}, \mathrm{CDCl}_{3}\right) \delta$ $169.9\left(C_{6}\right), 164.7\left(C_{8}\right), 149.7\left(C_{9}\right), 148.3\left(C_{13}\right), 137.3\left(C_{11}\right), 136.0\left(C_{15}\right), 129.3\left(2 C_{16}\right), 128.7\left(C_{18}\right), 128.6$ $\left(2 C_{17}\right), 126.3\left(C_{12}\right), 122.2\left(C_{10}\right), 78.2\left(C_{14}\right), 58.6\left(C_{4}\right), 56.6\left(C_{1}\right), 43.9\left(C_{5}\right), 40.5\left(C_{7}\right), 20.8\left(C_{2}\right), 20.3\left(C_{3}\right)$. HRMS calculated for $\mathrm{C}_{20} \mathrm{H}_{23} \mathrm{~N}_{4} \mathrm{O}_{3}[\mathrm{M}+\mathrm{H}]^{+}: 367.1770$; found: 367.1752 . [ $\left.\alpha\right]_{\mathrm{D}}:-36.9^{\circ}$ (6,4 mg/mL, MeOH).

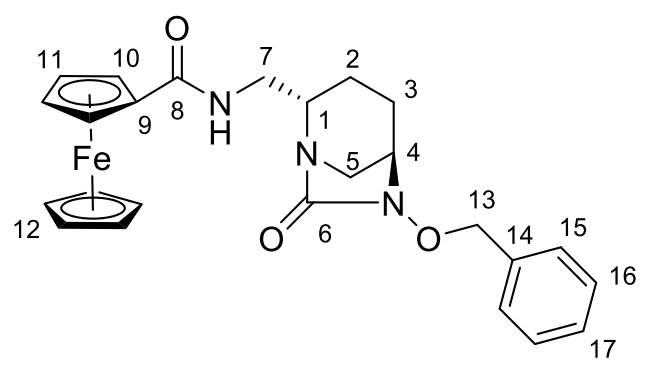

Compound 4f. Following the general procedure for the traceless Staudinger ligation, compound $\mathbf{4} \mathbf{f}$ was obtained as an orange foam (140 mg, 85\%) after purification by flash chromatography using cyclohexane/ethyl acetate (2/8) as eluent, starting from azido $3(100 \mathrm{mg}, 0.35 \mathrm{mmol})$ and ester phenylphosphine $2 \mathrm{f}(171 \mathrm{mg}, 0.35 \mathrm{mmol})$ in THF/ $\mathrm{H}_{2} \mathrm{O}(5,5 \mathrm{~mL}) .{ }^{1} \mathrm{H}$ NMR $\left(500 \mathrm{MHz}, \mathrm{CDCl}_{3}\right) \delta 7.43-7.40$ $\left(m, 2 \mathrm{H}, \mathrm{H}_{15}\right), 7.38-7.32\left(\mathrm{~m}, 3 \mathrm{H}, \mathrm{H}_{16,17}\right), 6.28$ (bs, $\left.1 \mathrm{H}, \mathrm{NH}\right), 5.04$ (d, J=11.5 Hz, 1H, $\left.\mathrm{H}_{13}\right), 4.90$ (d, J = 11.5 $\left.\mathrm{Hz}, 1 \mathrm{H}, \mathrm{H}_{13}\right), 4.70-4.67\left(\mathrm{~m}, 2 \mathrm{H}, \mathrm{H}_{10}\right), 4.31-4.30\left(\mathrm{~m}, 2 \mathrm{H}, \mathrm{H}_{11}\right), 4.18\left(\mathrm{~s}, 5 \mathrm{H}, \mathrm{H}_{12}\right), 3.62-3.49\left(\mathrm{~m}, 3 \mathrm{H}, \mathrm{H}_{1}\right.$, 7), $3.35-3.33\left(\mathrm{~m}, 1 \mathrm{H}, \mathrm{H}_{4}\right), 3.03\left(\mathrm{~d}, J=11.8 \mathrm{~Hz}, 1 \mathrm{H}, \mathrm{H}_{5}\right), 2.89\left(\mathrm{bd}, J=11.4 \mathrm{~Hz}, 1 \mathrm{H}, \mathrm{H}_{5}\right), 2.06-1.98(\mathrm{~m}, 2 \mathrm{H}$, $\left.\mathrm{H}_{2,3}\right), 1.68-1.61\left(\mathrm{~m}, 1 \mathrm{H}, \mathrm{H}_{3}\right), 1.48-1.43\left(\mathrm{~m}, 1 \mathrm{H}, \mathrm{H}_{2}\right) .{ }^{13} \mathrm{C} \mathrm{NMR}\left(126 \mathrm{MHz}, \mathrm{CDCl}_{3}\right) \delta 170.7\left(\mathrm{C}_{8}\right), 170.1$ $\left(C_{6}\right), 135.9\left(C_{14}\right), 129.2\left(2 C_{15}\right), 128.8\left(C_{17}\right), 128.6\left(2 C_{16}\right), 78.3\left(C_{13}\right), 75.9\left(C_{9}\right), 70.5\left(2 C_{11}\right), 69.8\left(5 C_{12}\right), 68.5$ $\left(C_{10}\right), 68.2\left(C_{10}\right), 58.7\left(C_{4}\right), 56.7\left(C_{1}\right), 43.8\left(C_{5}\right), 40.4\left(C_{7}\right), 20.7\left(C_{2}\right), 20.2\left(C_{3}\right)$. HRMS calculated for

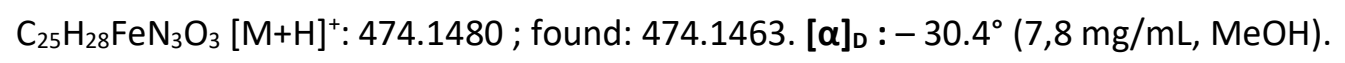

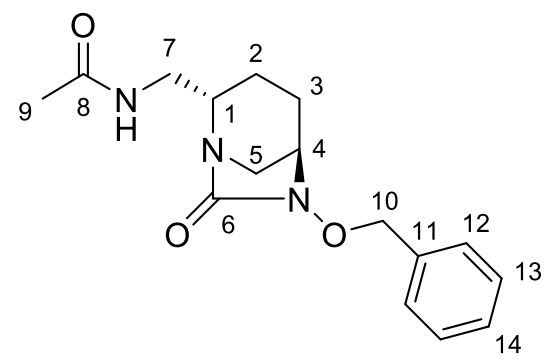

Compound $\mathbf{4 g}$. Following the general procedure for the traceless Staudinger ligation, compound $\mathbf{4 g}$ was obtained as a white foam (102 mg, 97\%) after purification by flash chromatography using $\mathrm{DCM} / \mathrm{MeOH}(96 / 4)$ as eluent, starting from azido 3 (100 mg, $0.35 \mathrm{mmol}$ ) and ester phenylphosphine $2 \mathrm{~g}$ (112 mg, $0.35 \mathrm{mmol})$ in THF/ $\mathrm{H}_{2} \mathrm{O}(5,5 \mathrm{~mL}) .{ }^{1} \mathrm{H}$ NMR (500 MHz, $\left.\mathrm{CDCl}_{3}\right) \delta 7.38-7.36\left(\mathrm{~m}, 2 \mathrm{H}, \mathrm{H}_{12}\right), 7.34$ 
$-7.28\left(\mathrm{~m}, 3 \mathrm{H}, \mathrm{H}_{13,14}\right), 6.40$ (bs, $\left.1 \mathrm{H}, \mathrm{NH}\right), 4.98\left(\mathrm{~d}, J=11.4 \mathrm{~Hz}, 1 \mathrm{H}, \mathrm{H}_{10}\right), 4.85\left(\mathrm{~d}, J=11.4 \mathrm{~Hz}, 1 \mathrm{H}, \mathrm{H}_{10}\right), 3.45$ $-3.37\left(\mathrm{~m}, 3 \mathrm{H}, \mathrm{H}_{1,7}\right), 3.32-3.30\left(\mathrm{~m}, 1 \mathrm{H}, \mathrm{H}_{4}\right), 2.96\left(\mathrm{~d}, J=11.9 \mathrm{~Hz}, 1 \mathrm{H}, \mathrm{H}_{5}\right), 2.81\left(\mathrm{bd}, J=11.7 \mathrm{~Hz}, 1 \mathrm{H}, \mathrm{H}_{5}\right)$, $1.98-1.93\left(\mathrm{~m}, 2 \mathrm{H}, \mathrm{H}_{2}, 3\right), 1.91\left(\mathrm{~s}, 3 \mathrm{H}, \mathrm{H}_{9}\right), 1.61-1.54\left(\mathrm{~m}, 1 \mathrm{H}, \mathrm{H}_{3}\right), 1.37-1.34\left(\mathrm{~m}, 1 \mathrm{H}, \mathrm{H}_{2}\right) .{ }^{13} \mathrm{C}$ NMR $(126$ $\left.\mathrm{MHz}, \mathrm{CDCl}_{3}\right) \delta 170.5\left(\mathrm{C}_{8}\right), 169.9\left(\mathrm{C}_{6}\right), 135.7\left(\mathrm{C}_{11}\right), 129.2\left(2 \mathrm{C}_{12}\right), 128.7\left(\mathrm{C}_{14}\right), 128.5\left(2 \mathrm{C}_{13}\right), 78.2\left(\mathrm{C}_{10}\right), 58.5$ $\left(C_{4}\right), 56.5\left(C_{1}\right), 43.8\left(C_{5}\right), 40.3\left(C_{7}\right), 23.1\left(C_{9}\right), 20.6\left(C_{2}\right), 20.0\left(C_{3}\right)$. HRMS calculated for $\mathrm{C}_{16} \mathrm{H}_{22} \mathrm{~N}_{3} \mathrm{O}_{3}[\mathrm{M}+\mathrm{H}]^{+}$:

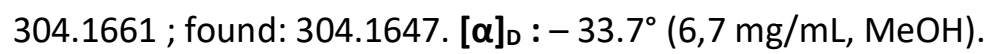

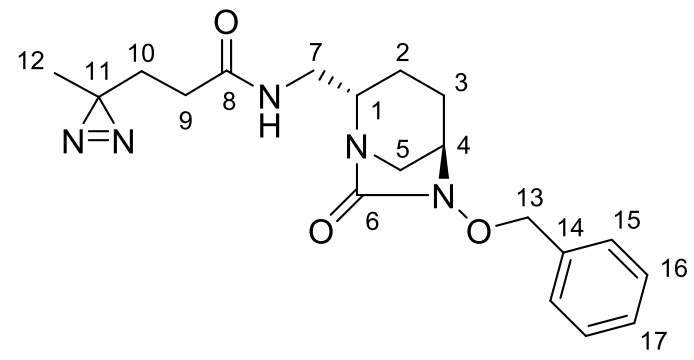

Compound $\mathbf{4 h}$. Following the general procedure for the traceless Staudinger ligation, compound $\mathbf{4 h}$ was obtained as a colorless oil (100 mg, 74\%) after purification by flash chromatography using cyclohexane/ethyl acetate (1/9) as eluent, starting from azido $3(105 \mathrm{mg}, 0.37 \mathrm{mmol})$ and ester phenylphosphine $2 \mathrm{~h}(142 \mathrm{mg}, 0.37 \mathrm{mmol})$ in THF/ $\mathrm{H}_{2} \mathrm{O}(5,5 \mathrm{~mL}) .{ }^{1} \mathrm{H} \mathbf{N M R}\left(500 \mathrm{MHz}, \mathrm{CDCl}_{3}\right) \delta 7.39-7.35$ $\left(\mathrm{m}, 2 \mathrm{H}, \mathrm{H}_{15}\right), 7.34-7.28\left(\mathrm{~m}, 3 \mathrm{H}, \mathrm{H}_{16,17}\right), 6.39$ (bs, $\left.1 \mathrm{H}, \mathrm{NH}\right), 4.99$ (d, J = $\left.11.4 \mathrm{~Hz}, 1 \mathrm{H}, \mathrm{H}_{13}\right), 4.85$ (d, J = 11.4 $\left.\mathrm{Hz}, 1 \mathrm{H}, \mathrm{H}_{13}\right), 3.43-3.38\left(\mathrm{~m}, 3 \mathrm{H}, \mathrm{H}_{1,7}\right), 3.32-3.30\left(\mathrm{~m}, 1 \mathrm{H}, \mathrm{H}_{4}\right), 2.95\left(\mathrm{~d}, J=11.9 \mathrm{~Hz}, 1 \mathrm{H}, \mathrm{H}_{5}\right), 2.82(\mathrm{bd}, J=$ $\left.11.6 \mathrm{~Hz}, 1 \mathrm{H}, \mathrm{H}_{5}\right), 2.00-1.90\left(\mathrm{~m}, 4 \mathrm{H}, \mathrm{H}_{2,3,9}\right), 1.67\left(\mathrm{t}, J=7.8 \mathrm{~Hz}, 2 \mathrm{H}, \mathrm{H}_{10}\right), 1.62-1.54\left(\mathrm{~m}, 1 \mathrm{H}, \mathrm{H}_{3}\right), 1.39-$ $1.34\left(\mathrm{~m}, 1 \mathrm{H}, \mathrm{H}_{2}\right), 0.96\left(\mathrm{~s}, 3 \mathrm{H}, \mathrm{H}_{12}\right) .{ }^{13} \mathrm{C}$ NMR $\left(126 \mathrm{MHz}, \mathrm{CDCl}_{3}\right) \delta 171.7\left(\mathrm{C}_{8}\right), 169.9\left(\mathrm{C}_{6}\right), 135.7\left(\mathrm{C}_{14}\right), 129.2$ $\left(2 C_{15}\right), 128.7\left(C_{17}\right), 128.5\left(2 C_{16}\right), 78.2\left(C_{13}\right), 58.5\left(C_{4}\right), 56.4\left(C_{1}\right), 43.8\left(C_{5}\right), 40.3\left(C_{7}\right), 30.5\left(C_{9}\right), 30.1\left(C_{10}\right)$, $25.4\left(C_{11}\right), 20.6\left(C_{2}\right), 20.0\left(C_{3}\right), 19.8\left(C_{12}\right)$. HRMS calculated for $\mathrm{C}_{19} \mathrm{H}_{26} \mathrm{~N}_{5} \mathrm{O}_{3}[\mathrm{M}+\mathrm{H}]^{+}: 372.2036$; found: 372.2020. $[\alpha]_{\mathrm{D}}:-29.8^{\circ}(6,1 \mathrm{mg} / \mathrm{mL}, \mathrm{MeOH})$.

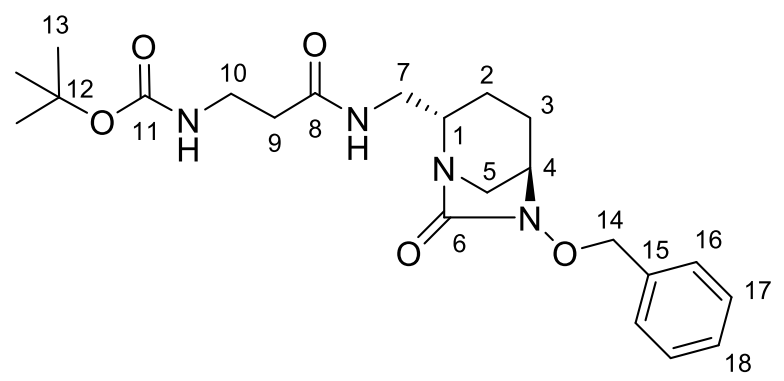

Compound 4i. Following the general procedure for the traceless Staudinger ligation, compound $\mathbf{4 i}$ was obtained as a white foam (100 mg, 66\%) after purification by flash chromatography using DCM/MeOH 
(96/4) as eluent, starting from azido $3(100 \mathrm{mg}, 0.35 \mathrm{mmol})$ and ester phenylphosphine 2i (157 mg, $0.35 \mathrm{mmol})$ in THF/ $\mathrm{H}_{2} \mathrm{O}(5,5 \mathrm{~mL}) .{ }^{1} \mathrm{H}$ NMR $\left(500 \mathrm{MHz}, \mathrm{CDCl}_{3}\right) \delta 7.39-7.36\left(\mathrm{~m}, 2 \mathrm{H}, \mathrm{H}_{16}\right), 7.35-7.29(\mathrm{~m}$, $\left.3 \mathrm{H}, \mathrm{H}_{17,18}\right), 6.44$ (bs, $1 \mathrm{H}, \mathrm{NH}$ ), 5.29 (bs, $1 \mathrm{H}, \mathrm{NH}_{\text {Boc }}$ ), 4.99 (d, J $\left.=11.5 \mathrm{~Hz}, 1 \mathrm{H}, \mathrm{H}_{14}\right), 4.85$ (d, J = $11.5 \mathrm{~Hz}, 1 \mathrm{H}$, $\left.\mathrm{H}_{14}\right), 3.45-3.39\left(\mathrm{~m}, 3 \mathrm{H}, \mathrm{H}_{1,7}\right), 3.37-3.30\left(\mathrm{~m}, 3 \mathrm{H}, \mathrm{H}_{4}, 10\right), 2.94\left(\mathrm{~d}, J=11.8 \mathrm{~Hz}, 1 \mathrm{H}, \mathrm{H}_{5}\right), 2.82(\mathrm{bd}, J=11.4$ $\left.\mathrm{Hz}, 1 \mathrm{H}, \mathrm{H}_{5}\right), 2.34\left(\mathrm{t}, J=5.8 \mathrm{~Hz}, 2 \mathrm{H}, \mathrm{H}_{9}\right), 1.99-1.91\left(\mathrm{~m}, 2 \mathrm{H}, \mathrm{H}_{2,3}\right), 1.61-1.54\left(\mathrm{~m}, 1 \mathrm{H}, \mathrm{H}_{3}\right), 1.38(\mathrm{~s}, 9 \mathrm{H}$, $\left.\mathrm{H}_{13}\right), 1.35-1.33\left(\mathrm{~m}, 1 \mathrm{H}, \mathrm{H}_{2}\right) .{ }^{13} \mathrm{C} \mathrm{NMR}\left(126 \mathrm{MHz}, \mathrm{CDCl}_{3}\right) \delta 171.9\left(\mathrm{C}_{8}\right), 169.9\left(\mathrm{C}_{6}\right), 156.0\left(\mathrm{C}_{11}\right), 135.8\left(\mathrm{C}_{15}\right)$, $129.2\left(2 C_{16}\right), 128.8\left(C_{18}\right), 128.6\left(2 C_{17}\right), 79.1\left(C_{12}\right), 78.2\left(C_{14}\right), 58.5\left(C_{4}\right), 56.5\left(C_{1}\right), 43.7\left(C_{5}\right), 40.2\left(C_{7}\right), 36.8$ $\left(C_{10}\right), 36.1\left(C_{9}\right), 28.4\left(3 C_{13}\right), 20.6\left(C_{2}\right), 20.1\left(C_{3}\right)$. HRMS calculated for $\mathrm{C}_{22} \mathrm{H}_{33} \mathrm{~N}_{4} \mathrm{O}_{5}[\mathrm{M}+\mathrm{H}]^{+}: 433.2451$; found: $433.2433 .[\alpha]_{\mathrm{D}}:-32.4^{\circ}(6,8 \mathrm{mg} / \mathrm{mL}, \mathrm{MeOH})$.

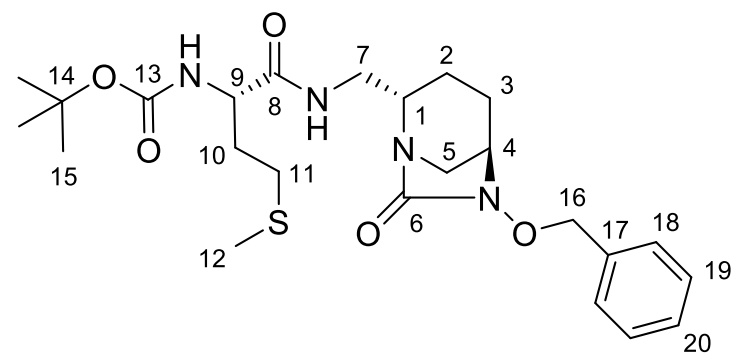

Compound $\mathbf{4 j}$. Following the general procedure for the traceless Staudinger ligation, compound $\mathbf{4 j}$ was obtained as a white foam (94 mg, 55\%) after purification by flash chromatography using cyclohexane/ethyl acetate (3/7) as eluent, starting from azido $3(100 \mathrm{mg}, 0.35 \mathrm{mmol})$ and ester phenylphosphine $2 \mathbf{j}$ (178 mg, $0.35 \mathrm{mmol}$ ) in THF/ $\mathrm{H}_{2} \mathrm{O}(5,5 \mathrm{~mL}) .{ }^{1} \mathrm{H}$ NMR $\left(500 \mathrm{MHz}, \mathrm{CDCl}_{3}\right) \delta 7.40-7.36$ $\left(\mathrm{m}, 2 \mathrm{H}, \mathrm{H}_{18}\right), 7.36-7.30\left(\mathrm{~m}, 3 \mathrm{H}, \mathrm{H}_{19}, 20\right), 6.76(\mathrm{bs}, 1 \mathrm{H}, \mathrm{NH}), 5.33\left(\mathrm{bd}, J=7.5 \mathrm{~Hz}, 1 \mathrm{H}, \mathrm{NH}_{\text {Boc }}\right), 5.01(\mathrm{~d}, J=$ $\left.11.5 \mathrm{~Hz}, 1 \mathrm{H}, \mathrm{H}_{16}\right), 4.86\left(\mathrm{~d}, J=11.5 \mathrm{~Hz}, 1 \mathrm{H}, \mathrm{H}_{16}\right), 4.22-4.21\left(\mathrm{~m}, 1 \mathrm{H}, \mathrm{H}_{9}\right), 3.49-3.41\left(\mathrm{~m}, 3 \mathrm{H}, \mathrm{H}_{1,7}\right), 3.29-$ $3.28\left(\mathrm{~m}, 1 \mathrm{H}, \mathrm{H}_{4}\right), 2.92\left(\mathrm{~d}, J=11.9 \mathrm{~Hz}, 1 \mathrm{H}, \mathrm{H}_{5}\right), 2.81\left(\mathrm{bd}, J=11.4 \mathrm{~Hz}, 1 \mathrm{H}, \mathrm{H}_{5}\right), 2.51\left(\mathrm{t}, J=7.5 \mathrm{~Hz}, 2 \mathrm{H}, \mathrm{H}_{11}\right)$, $2.08-2.00\left(m, 4 \mathrm{H}, \mathrm{H}_{10,12}\right), 1.99-1.92\left(\mathrm{~m}, 2 \mathrm{H}, \mathrm{H}_{2,3}\right), 1.90-1.83\left(\mathrm{~m}, 1 \mathrm{H}, \mathrm{H}_{10}\right), 1.61-1.55\left(\mathrm{~m}, 1 \mathrm{H}, \mathrm{H}_{3}\right)$, $1.40\left(\mathrm{~s}, 9 \mathrm{H}, \mathrm{H}_{15}\right), 1.37-1.34\left(\mathrm{~m}, 1 \mathrm{H}, \mathrm{H}_{2}\right) .{ }^{13} \mathrm{C} \mathrm{NMR}\left(126 \mathrm{MHz}, \mathrm{CDCl}_{3}\right) \delta 171.9\left(\mathrm{C}_{8}\right), 169.8\left(\mathrm{C}_{6}\right), 155.5\left(\mathrm{C}_{13}\right)$, $135.9\left(C_{17}\right), 129.2\left(2 C_{18}\right), 128.7\left(C_{20}\right), 128.6\left(2 C_{19}\right), 80.0\left(C_{14}\right), 78.3\left(C_{16}\right), 58.5\left(C_{4}\right), 56.3\left(C_{1}\right), 53.8\left(C_{9}\right)$, 43.8 $\left(C_{5}\right), 40.3\left(C_{7}\right), 32.1\left(C_{10}\right), 30.2\left(C_{11}\right), 28.4\left(3 C_{15}\right), 20.6\left(C_{2}\right), 20.1\left(C_{3}\right), 15.4\left(C_{12}\right)$. HRMS calculated for

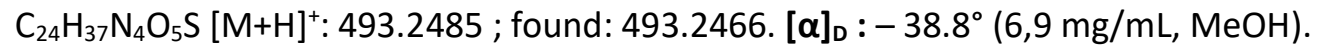




\section{Introduction of sodium sulfite}

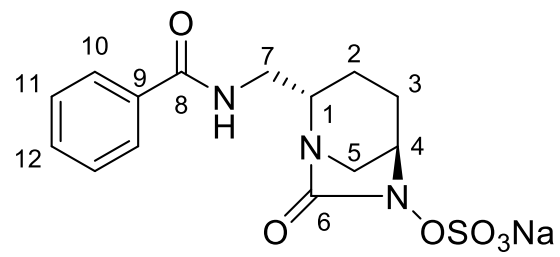

Compound 5a. $10 \mathrm{wt} \% \mathrm{Pd} / \mathrm{C}(21 \mathrm{mg}, 0.20 \mathrm{mmol}$ ) was added to a solution of compound $4 \mathrm{a}$ (72 $\mathrm{mg}$, $0.20 \mathrm{mmol}$ ) in $\mathrm{MeOH}(10 \mathrm{~mL})$ and the reaction mixture was stirred under $\mathrm{H}_{2}$ atmosphere for $48 \mathrm{~h}$ at room temperature. Palladium was removed by filtration through Celite ${ }^{\circledR}$ and the filtrate was concentrated. A sulfur trioxide-pyridine complex (185 $\mathrm{mg}, 1.18 \mathrm{mmol})$ was added to a solution of deprotected compound in pyridine $(3 \mathrm{~mL})$ and the reaction mixture was stirred for $2 \mathrm{~h}$ at room temperature. An additional amount of $\mathrm{SO}_{3}$-pyridine complex (62 $\mathrm{mg}, 0.39 \mathrm{mmol}$ ) was added and stirred for $16 \mathrm{~h}$ at room temperature. The pyridine was then removed under reduced pressure. The crude product was solubilized in water, filtered, eluted on Dowex- $\mathrm{Na}$ resin with $\mathrm{H}_{2} \mathrm{O}$ and lyophilized. The residue was dissolved in EtOH, filtered, and concentrated under vacuum. HPLC purification afforded compound 5 a as white powder (18 mg, 24\%). ${ }^{1} \mathbf{H}$ NMR $\left(500 \mathrm{MHz}, \mathrm{D}_{2} \mathrm{O}\right) \delta 7.83(\mathrm{~d}, J=7.8 \mathrm{~Hz}$, $\left.2 \mathrm{H}, \mathrm{H}_{10}\right), 7.67\left(\mathrm{t}, J=7.4 \mathrm{~Hz}, 1 \mathrm{H}, \mathrm{H}_{12}\right), 7.58\left(\mathrm{t}, J=7.6 \mathrm{~Hz}, 2 \mathrm{H}, \mathrm{H}_{11}\right), 4.28\left(\mathrm{bs}, 1 \mathrm{H}, \mathrm{H}_{4}\right), 3.87(\mathrm{dd}, J=16.0,11.5$ $\left.\mathrm{Hz}, 1 \mathrm{H}, \mathrm{H}_{7}\right), 3.70-3.67\left(\mathrm{~m}, 2 \mathrm{H}, \mathrm{H}_{1,7}\right), 3.49\left(\mathrm{~d}, J=12.3 \mathrm{~Hz}, 1 \mathrm{H}, \mathrm{H}_{5}\right), 3.23\left(\mathrm{bd}, J=12.2 \mathrm{~Hz}, 1 \mathrm{H}, \mathrm{H}_{5}\right), 2.13-$ $2.09\left(m, 1 H, H_{3}\right), 2.01-1.93\left(m, 2 H, H_{2,3}\right), 1.73-1.67\left(m, 1 H, H_{2}\right) .{ }^{13} \mathrm{C} \mathrm{NMR}\left(126 \mathrm{MHz}, \mathrm{D}_{2} \mathrm{O}\right) \delta 171.0$ $\left(C_{8}\right), 170.7\left(C_{6}\right), 133.4\left(C_{9}\right), 132.3\left(C_{12}\right), 128.8\left(2 C_{11}\right), 127.1\left(2 C_{10}\right), 60.3\left(C_{4}\right), 57.4\left(C_{1}\right), 44.0\left(C_{5}\right), 40.7\left(C_{7}\right)$, $19.9\left(C_{2}\right), 19.0\left(C_{3}\right)$. HRMS calculated for $\mathrm{C}_{14} \mathrm{H}_{16} \mathrm{~N}_{3} \mathrm{O}_{6} \mathrm{~S}[\mathrm{M}-\mathrm{H}]=354.0760$; found: $354.0767 .[\alpha]_{D}:-51.6^{\circ}$ $(6,1 \mathrm{mg} / \mathrm{mL}, \mathrm{MeOH})$. HPLC purity $98.0 \%$ (BDS, acetonitrile/water $=0 / 100$ to $100 / 0$ in $15 \mathrm{~min}$, flow rate $=1.3 \mathrm{~mL} / \mathrm{min}, \mathrm{I}=220 \mathrm{~nm}$ ) $\mathrm{tR}=10.1 \mathrm{~min}$ 


\section{Phosphine-based redox catalysis ${ }^{7}$}<smiles>NC[C@@H]1CCC2CN1C(=O)N2Cc1ccccc1</smiles>

3<smiles>O=C(O)c1ccccc1</smiles>

$1 \mathrm{a}$

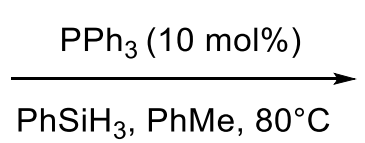

$\mathrm{PhSiH}_{3}, \mathrm{PhMe}, 80^{\circ} \mathrm{C}$<smiles>O=C(NC[C@@H]1CC[C@@H]2CN1C(=O)N2OCc1ccccc1)c1ccccc1</smiles>

$4 a$

\begin{tabular}{|c|c|c|c|c|}
\hline Entry & Time & Conditions $^{a}$ & Yield & Ratio 4a / O= $\mathrm{PPh}_{3}{ }^{b}$ \\
\hline $\mathbf{1}$ & $24 \mathrm{~h}$ & A & $17 \%$ & $3 / 1$ \\
\hline $\mathbf{2}$ & $48 \mathrm{~h}$ & A & $47 \%$ & $7 / 1$ \\
\hline $\mathbf{3}$ & $24 \mathrm{~h}$ & B & $51 \%$ & $12 / 1$ \\
\hline
\end{tabular}

a) Two conditions ( $\mathrm{A}$ and $\mathrm{B}$ ) were developed to remove 1a inseparable from 4a. $b)^{31} \mathbf{P ~ N M R ~}(202 \mathrm{MHz}, \mathrm{CDCl} 3) \delta-$ 29.1. HRMS calculated for $\mathrm{C}_{18} \mathrm{H}_{16} \mathrm{OP}[\mathrm{M}+\mathrm{H}]^{+}: 279.0939$; found: 279.0927.

Triphenylphosphine $(8 \mathrm{mg}, 0.03 \mathrm{mmol})$ and a solution of $\mathbf{3}(100 \mathrm{mg}, 0.35 \mathrm{mmol})$ in PhMe $(500 \mu \mathrm{L})$ were added sequentially to a mixture of $1 \mathrm{a}\left(35 \mathrm{mg}, 0.29 \mathrm{mmol}\right.$ ) and $\mathrm{PhSiH}_{3}(36 \mu \mathrm{L}, 0.29 \mathrm{mmol}$ ) in PhMe (500 $\mu \mathrm{L}$ ) at room temperature. The reaction was heated in an oil bath at $80^{\circ} \mathrm{C}$ and stirred for indicated time.

Conditions A: The mixture was diluted with ethyl acetate and washed with a saturated solution of sodium bicarbonate and brine, then dried over $\mathrm{MgSO}_{4}$, filtered, and concentrated under reduced pressure. The crude product was purified by flash chromatography cyclohexane/ethyl acetate $(3 / 7)$ to afford product $4 \mathrm{a}$ in mixture with triphenylphosphine oxyde.

Conditions B: The mixture was concentrated under vacuum and the crude product was purified by flash chromatography cyclohexane/ethyl acetate (3/7) to afford product $4 \mathbf{a}$ in mixture with triphenylphosphine oxyde and 1a. A final extraction step with dichloromethane and brine allows to remove 1a of the mixture.

\footnotetext{
${ }^{7}$ Kosal, A. D.; Wilson, E. E.; Ashfeld, B. L. Angew. Chem. Int. Ed. 2012, 51, 12036-12040.
} 


\section{Determination of Kinetic Parameters}

The kinetic parameter $k_{2} / K_{\mathrm{i}}$ for the carbamoylation of $\beta$-lactamases by avibactam, relebactam, and $\mathbf{5 a}$ was determined at $20^{\circ} \mathrm{C}$ in MES (100 mM; pH 6.4), as previously described. ${ }^{8}$ The reporter substrate was nitrocefin (100 $\mu \mathrm{M})$ for TEM-1, KPC-2, CTX-M-15, and AmpC clo or CENTA (100 $\mu \mathrm{M})$ for OXA-48. The variation in the molecular extinction coefficient was $15,200 \mathrm{M}^{-1} \mathrm{~cm}^{-1}$ at $486 \mathrm{~nm}$ for the hydrolysis of nitrocefin and 7,380 $\mathrm{M}^{-1} \mathrm{~cm}^{-1}$ at $415 \mathrm{~nm}$ for the hydrolysis of CENTA. Kinetics constants were deduced from a minimum of six progress curves obtained in a minimum of two independent experiments.

Data for avibactam and relebactam are from a previous study. ${ }^{9}$

${ }^{8}$ Ehmann, D. E.; Jahic, H.; Ross, P. L.; Gu, R. F.; Hu, J.; Kern, G.; Walkup, G. K.; Fisher, S. L. Proc. Natl. Acad. Sci. U. S. A. 2012, 109, $11663-11668$.

${ }^{9}$ Bouchet, F.; Atze, H.; Fonvielle, M.; Edoo, Z.; Arthur, M.; Ethève-Quelquejeu, M.; Iannazzo, L. J. Med. Chem. 2020, 63, 5257-5273. 


\section{NMR spectra of synthesized compounds}

${ }^{1} \mathrm{H} \mathrm{NMR}\left(\mathrm{CDCl}_{3}, 500 \mathrm{MHz}\right)$

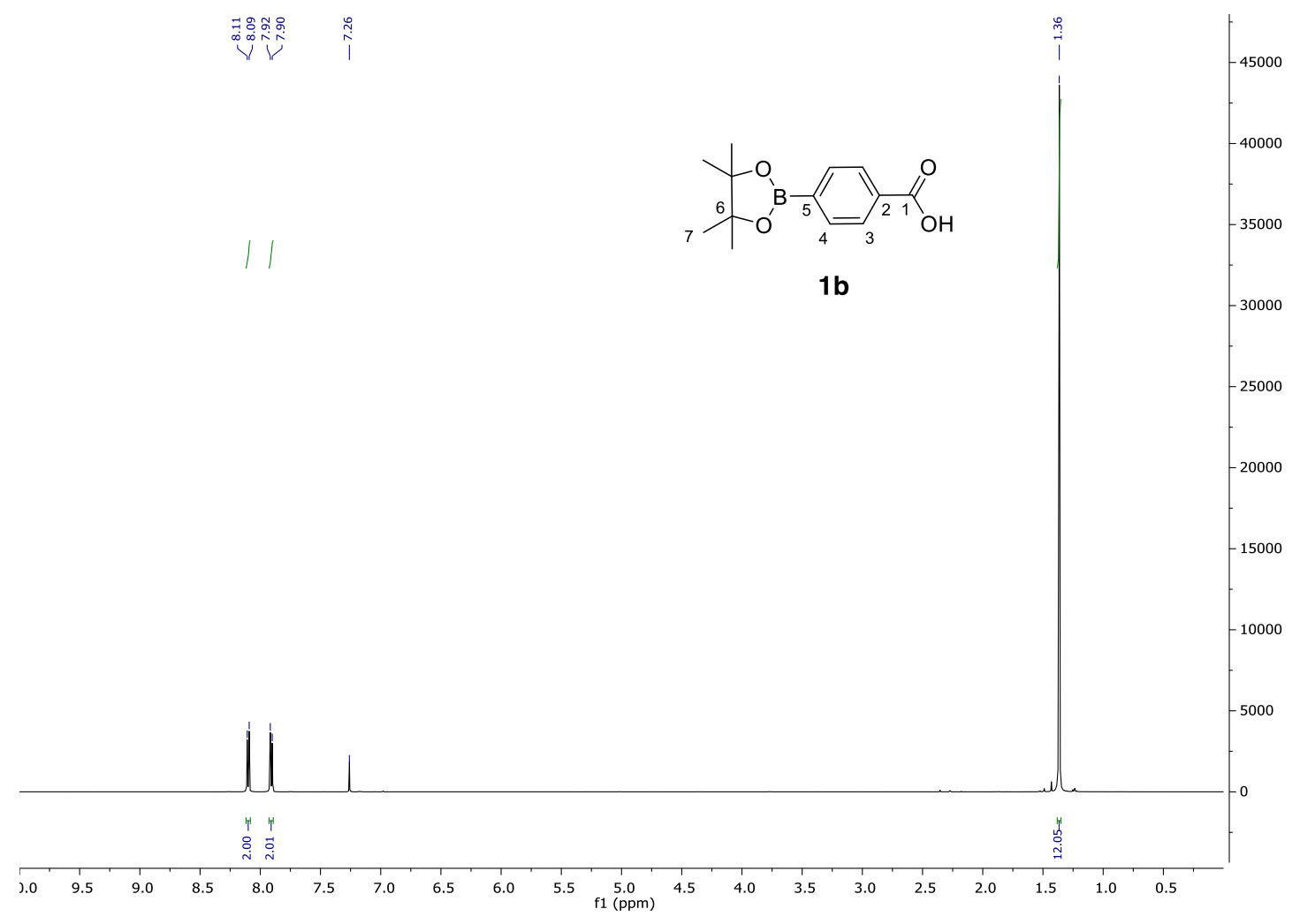

$\left.{ }^{13} \mathrm{C} \mathrm{NMR} \mathrm{(CDCl}, 126 \mathrm{MHz}\right)$

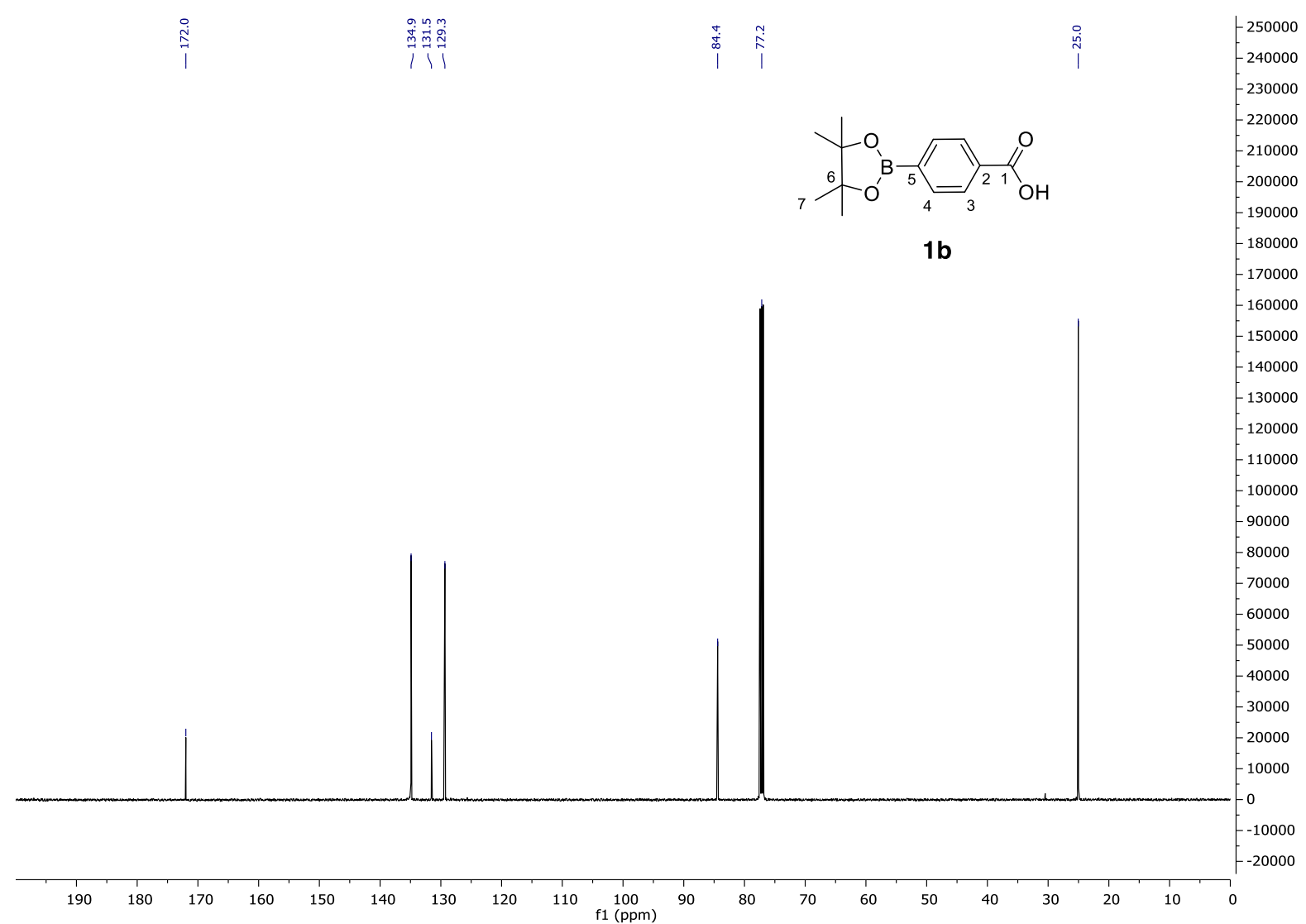


${ }^{1} \mathrm{H}$ NMR $\left(\mathrm{CDCl}_{3}, 500 \mathrm{MHz}\right)$

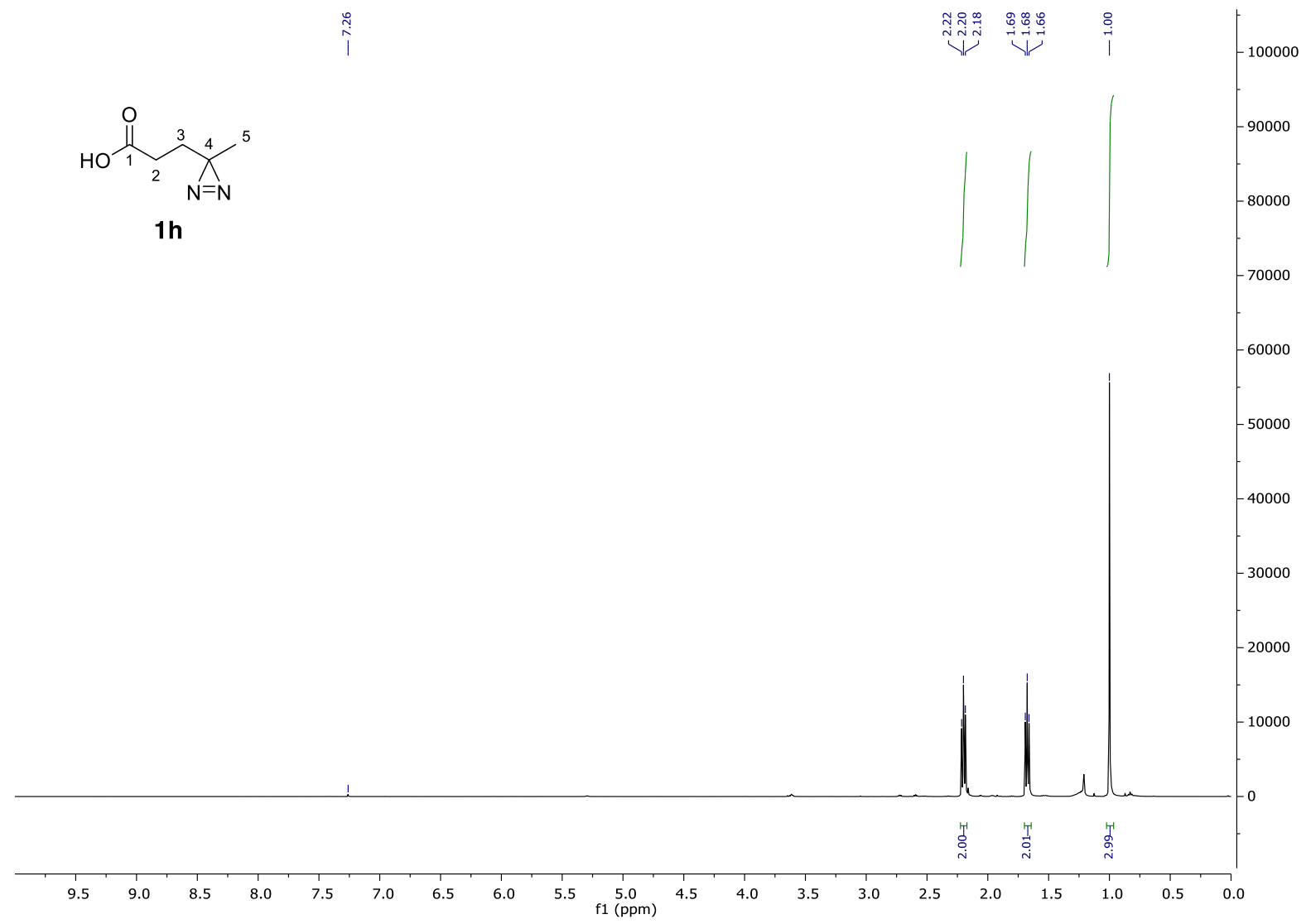

$\left.{ }^{13} \mathrm{C} \mathrm{NMR} \mathrm{(CDCl} 3,126 \mathrm{MHz}\right)$

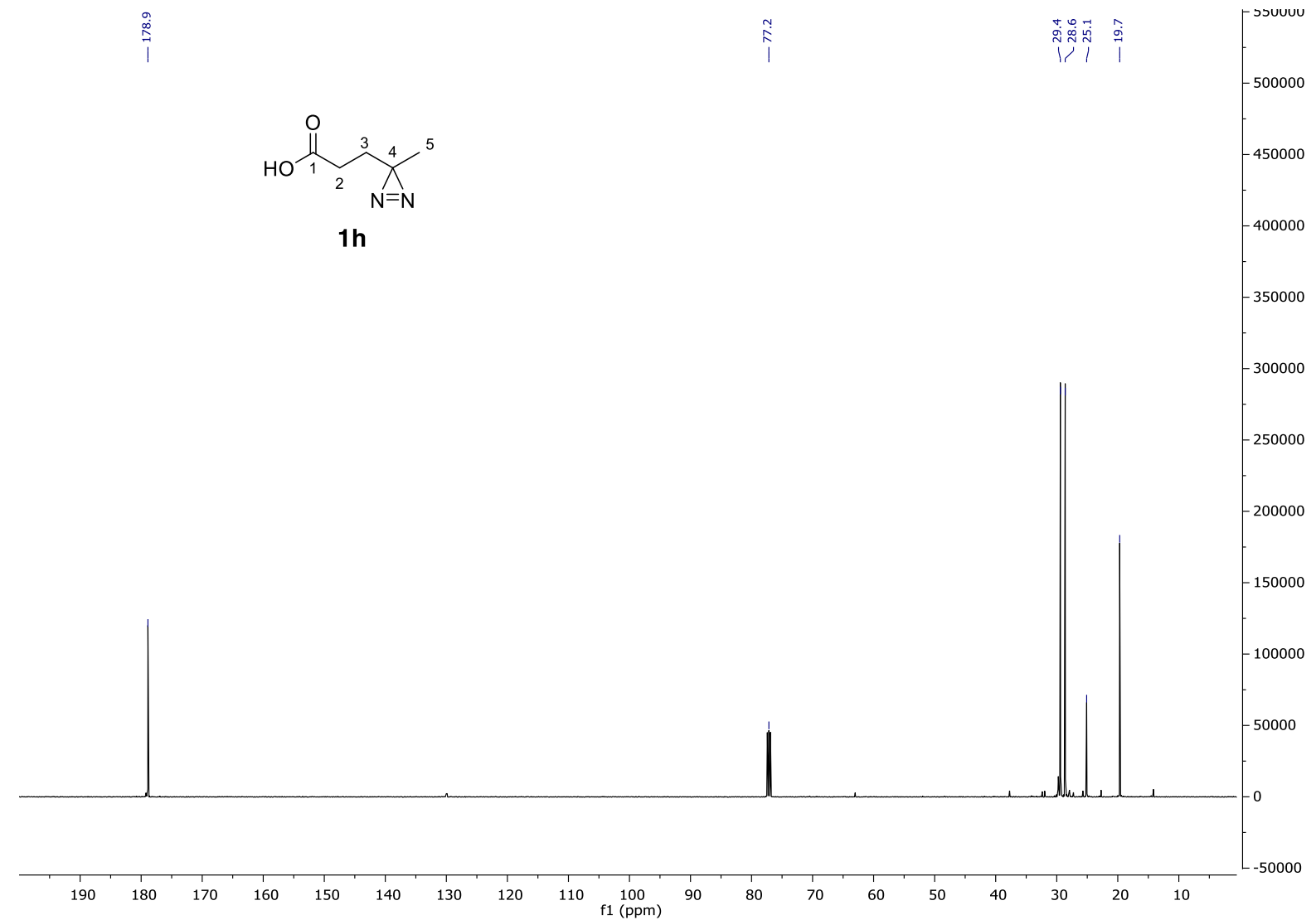


$\left.{ }^{1} \mathrm{H} \mathrm{NMR} \mathrm{(CDCl}{ }_{3}, 500 \mathrm{MHz}\right)$

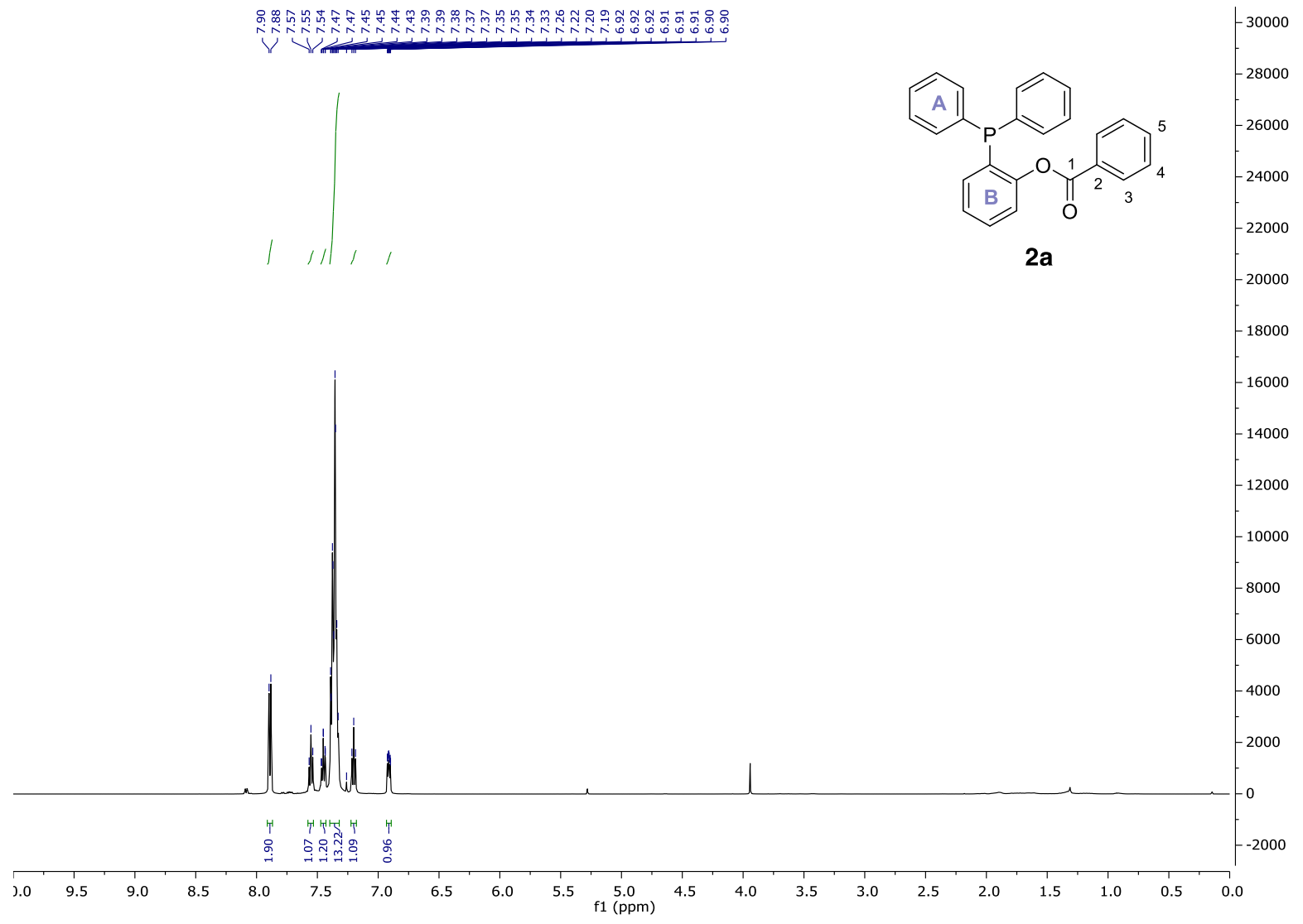

${ }^{13} \mathrm{C} \mathrm{NMR}\left(\mathrm{CDCl}_{3}, 126 \mathrm{MHz}\right)$

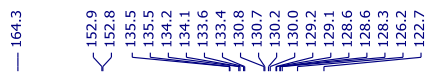
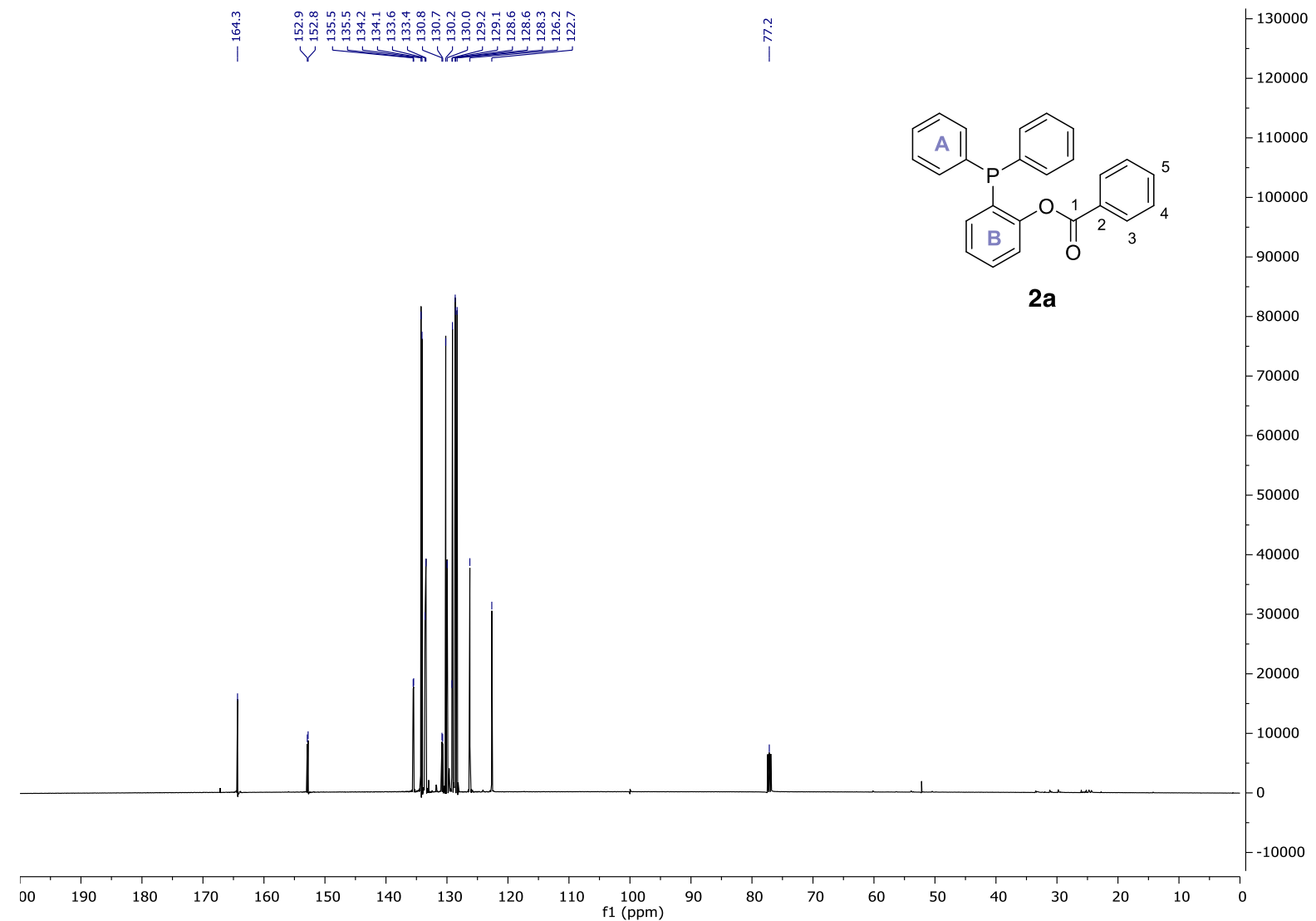
${ }^{31} \mathrm{P}$ NMR $\left(\mathrm{CDCl}_{3}, 202 \mathrm{MHz}\right)$

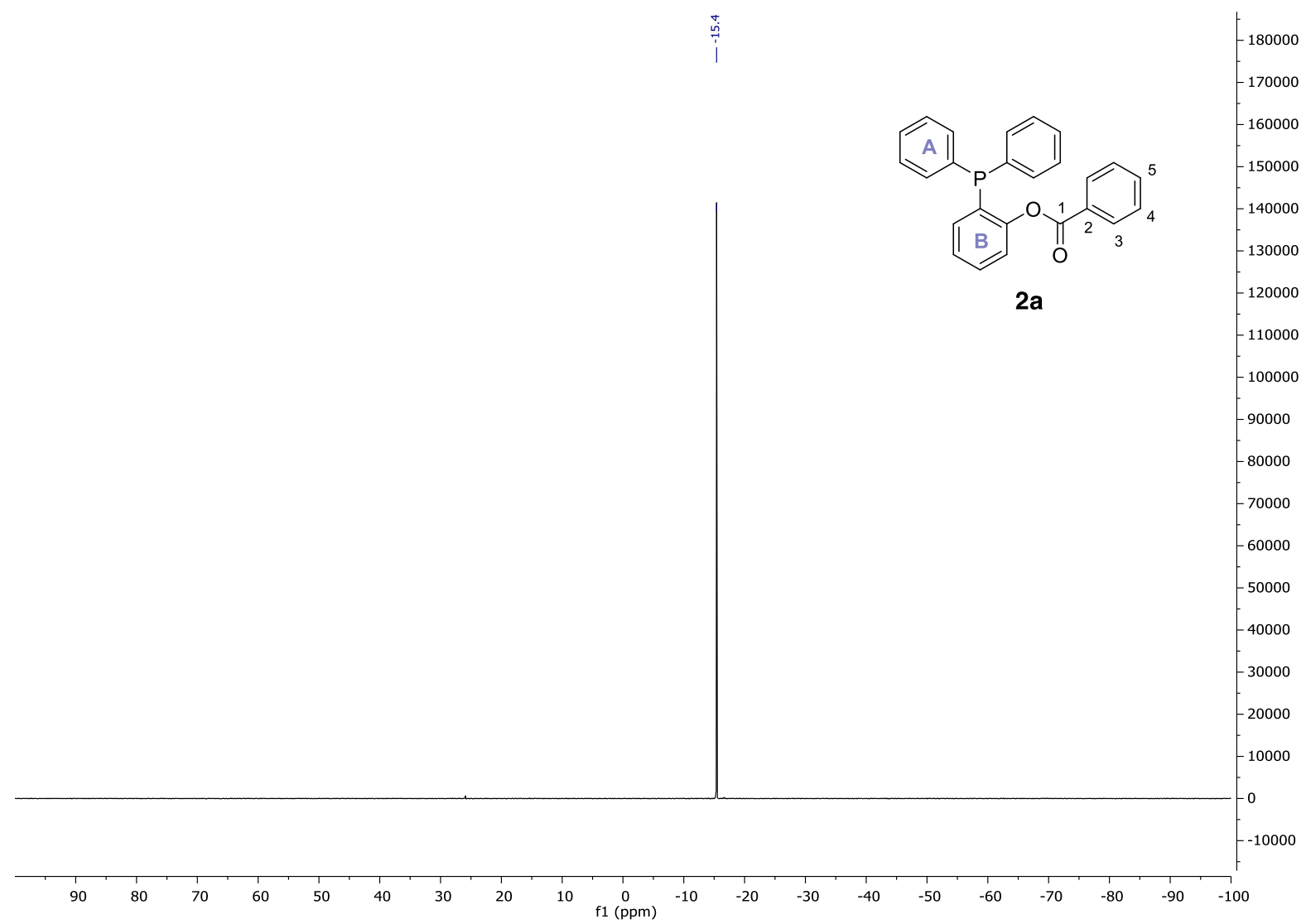

${ }^{1} \mathrm{H}$ NMR $\left(\mathrm{CDCl}_{3}, 500 \mathrm{MHz}\right)$

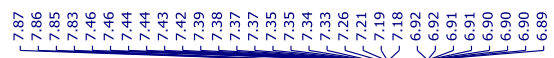

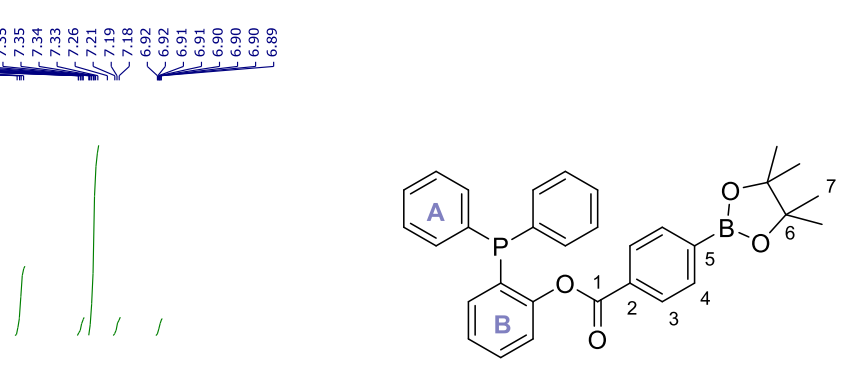

2b

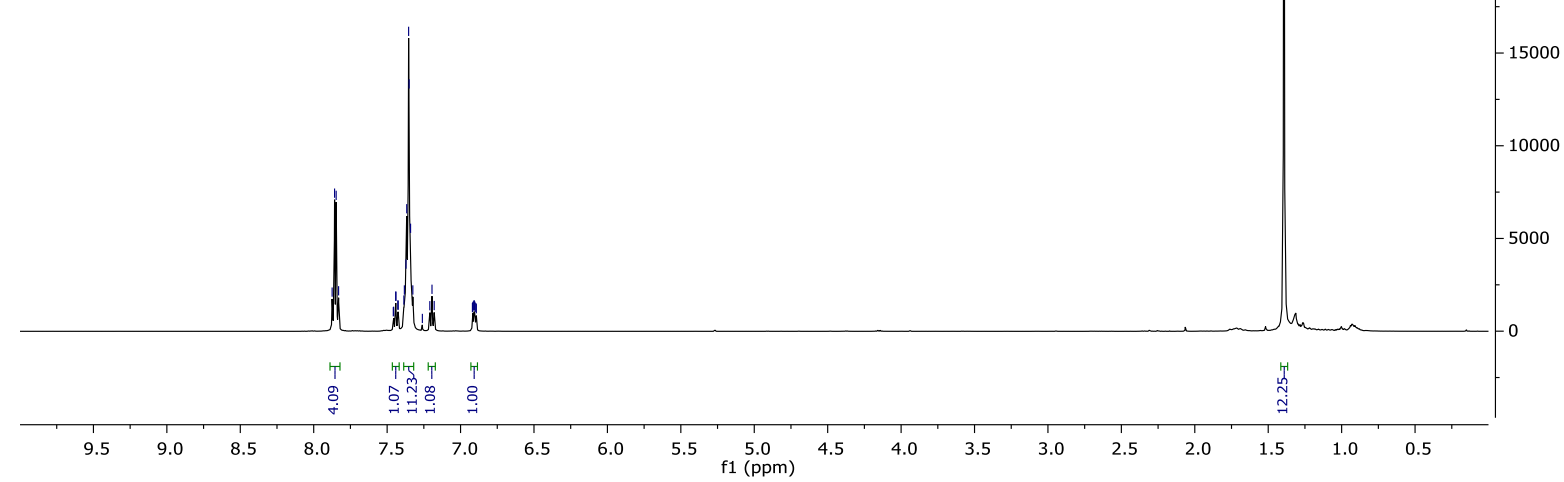


${ }^{13} \mathrm{C} \mathrm{NMR}\left(\mathrm{CDCl}_{3}, 126 \mathrm{MHz}\right)$

I
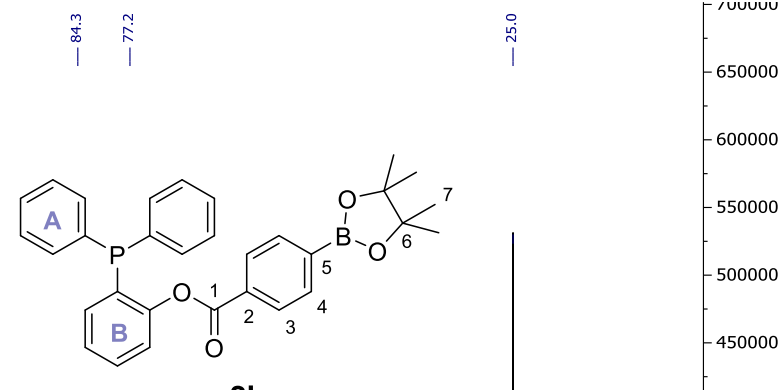

2b

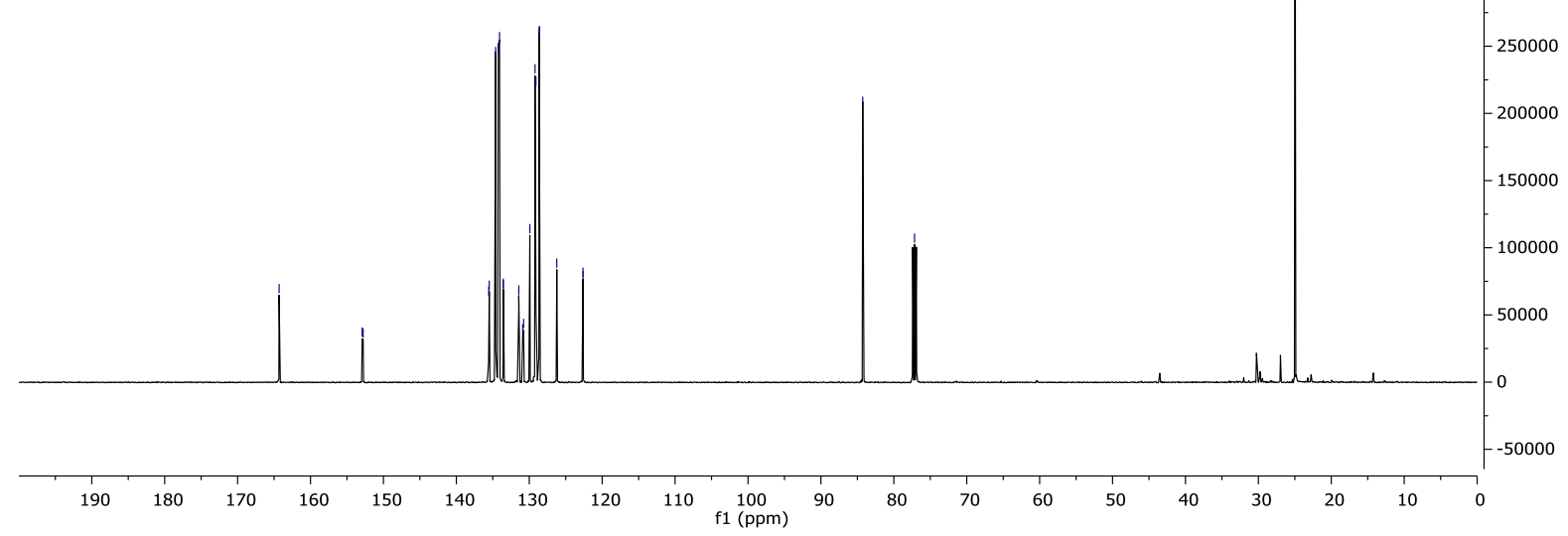

${ }^{31} \mathrm{P} \mathrm{NMR}\left(\mathrm{CDCl}_{3}, 202 \mathrm{MHz}\right)$

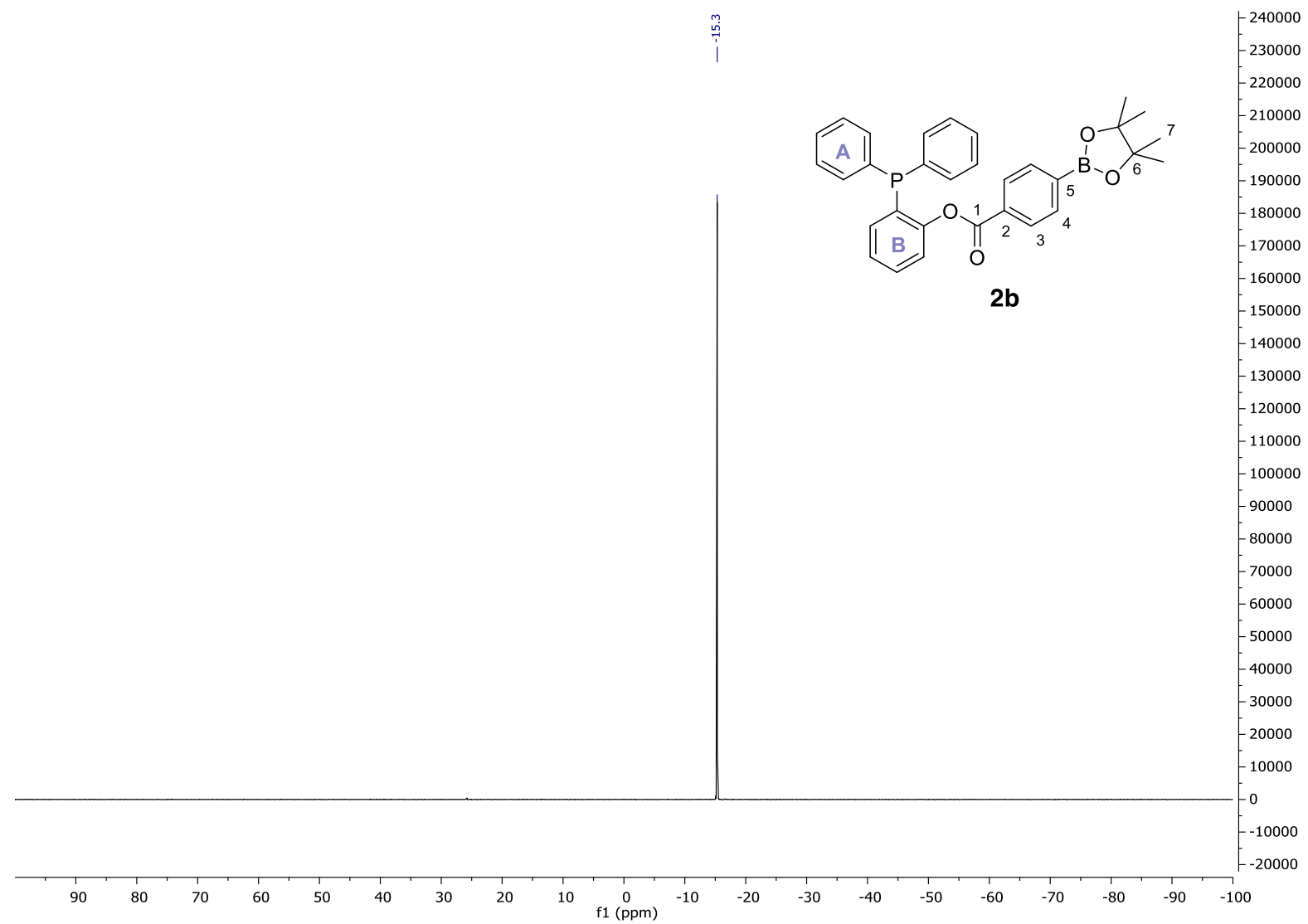


${ }^{1} \mathrm{H}$ NMR $\left(\mathrm{CDCl}_{3}, 500 \mathrm{MHz}\right)$

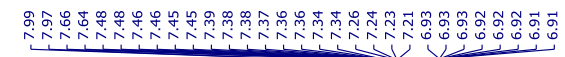

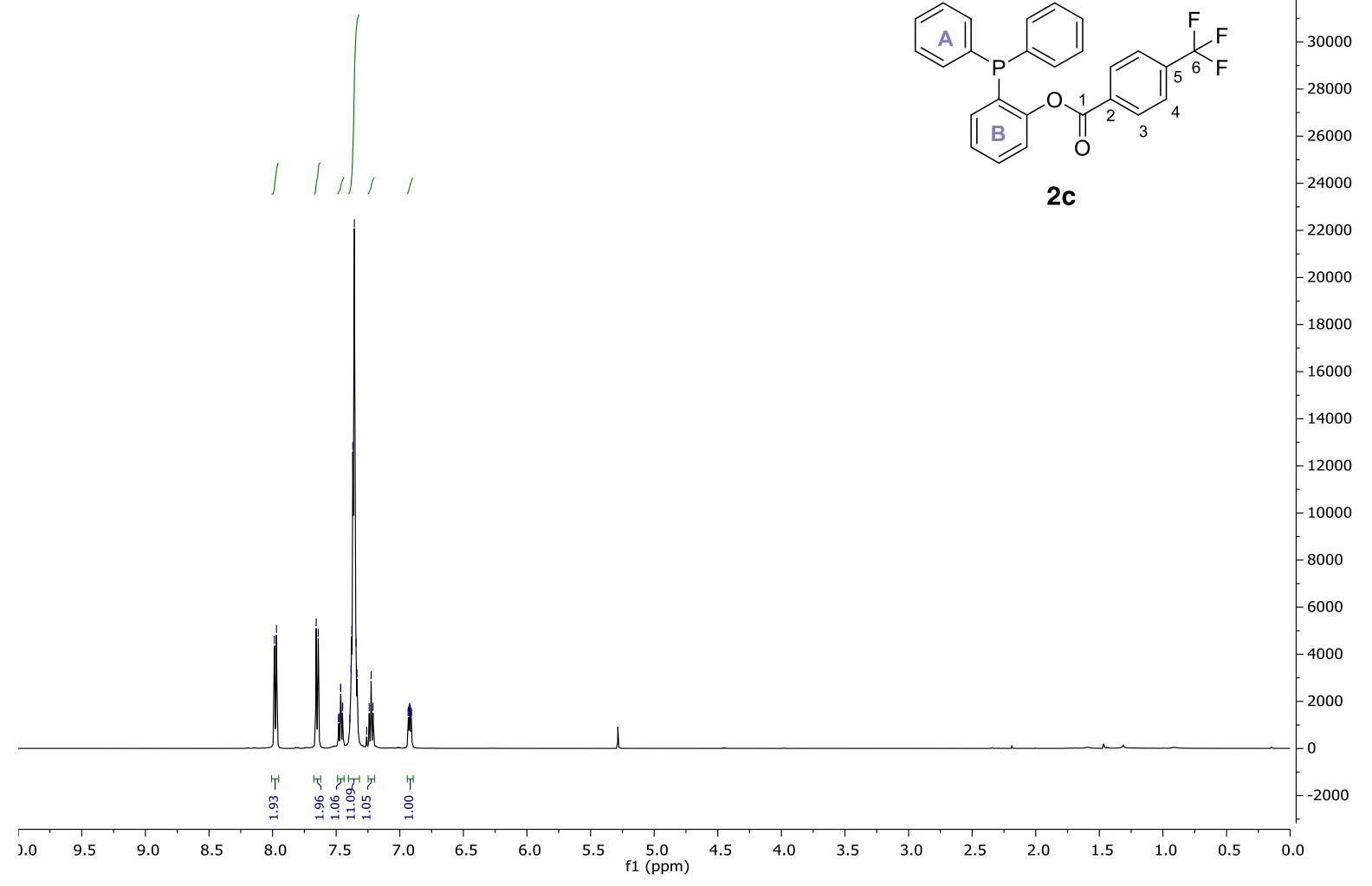

$\left.{ }^{13} \mathrm{C} \mathrm{NMR} \mathrm{(CDCl}, 126 \mathrm{MHz}\right)$

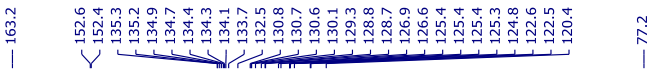

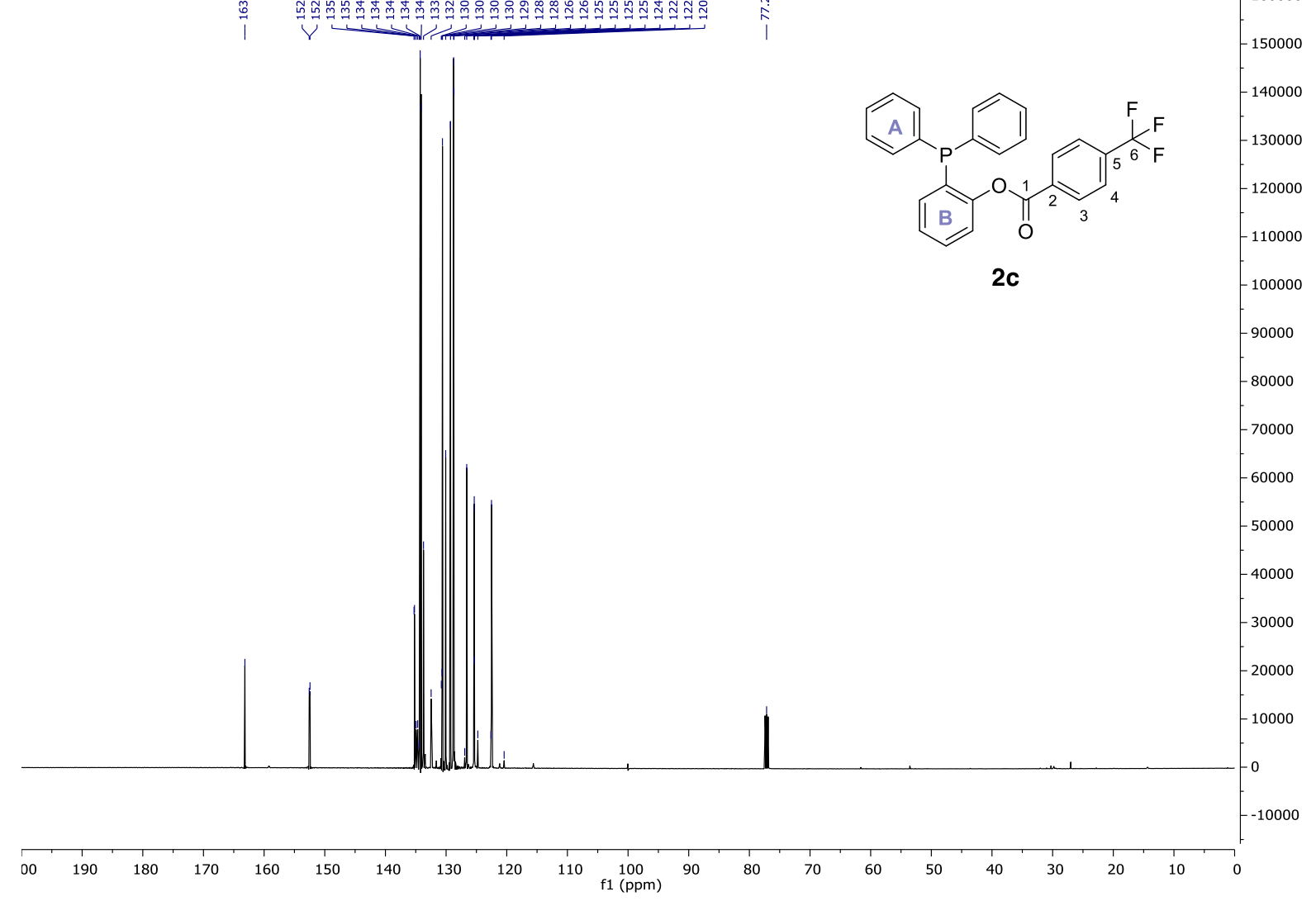


${ }^{31} \mathrm{P} \mathrm{NMR}\left(\mathrm{CDCl}_{3}, 202 \mathrm{MHz}\right)$

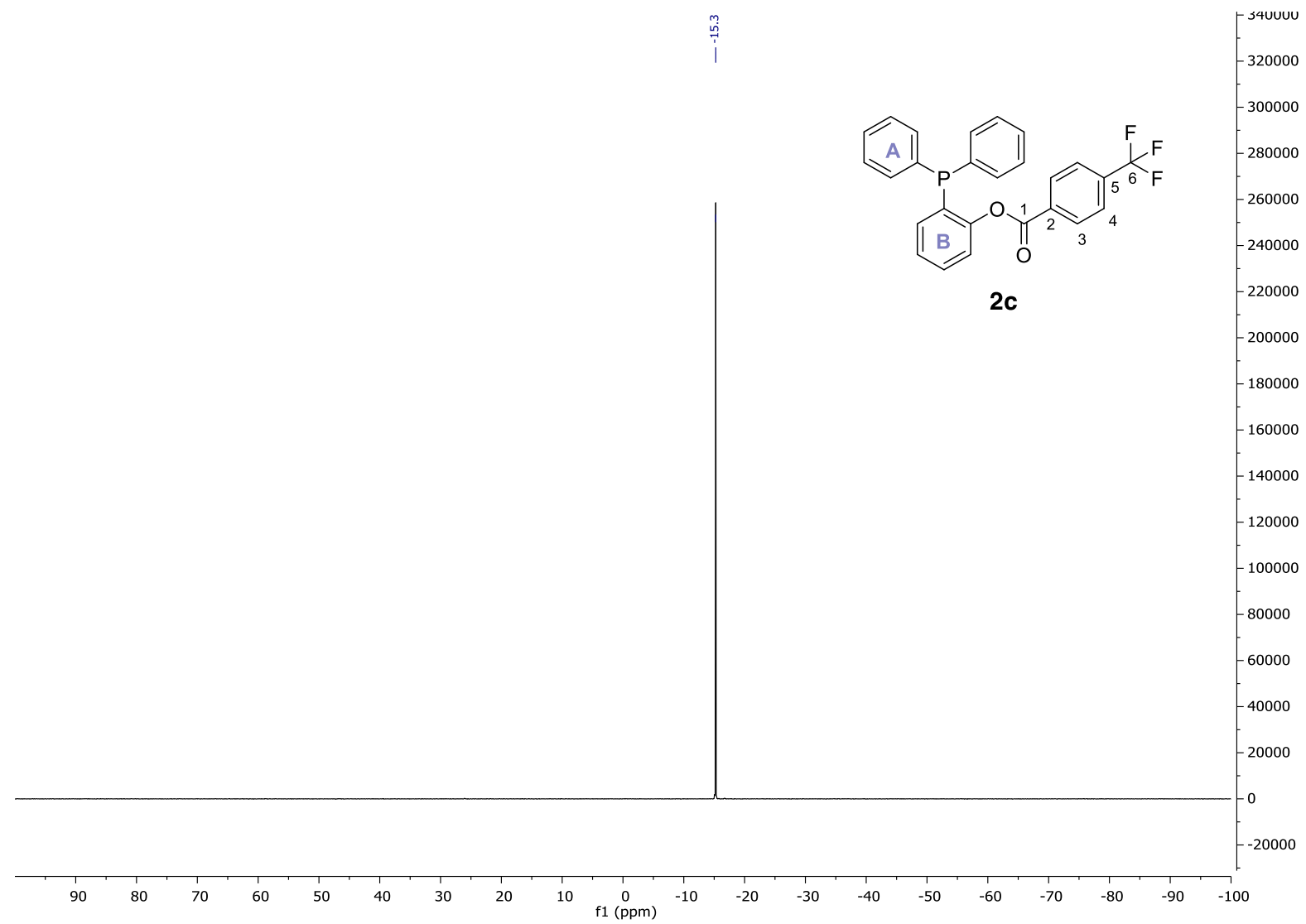

$\left.{ }^{1} \mathrm{H} \mathrm{NMR} \mathrm{(CDCl}{ }_{3}, 500 \mathrm{MHz}\right)$

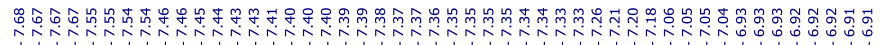

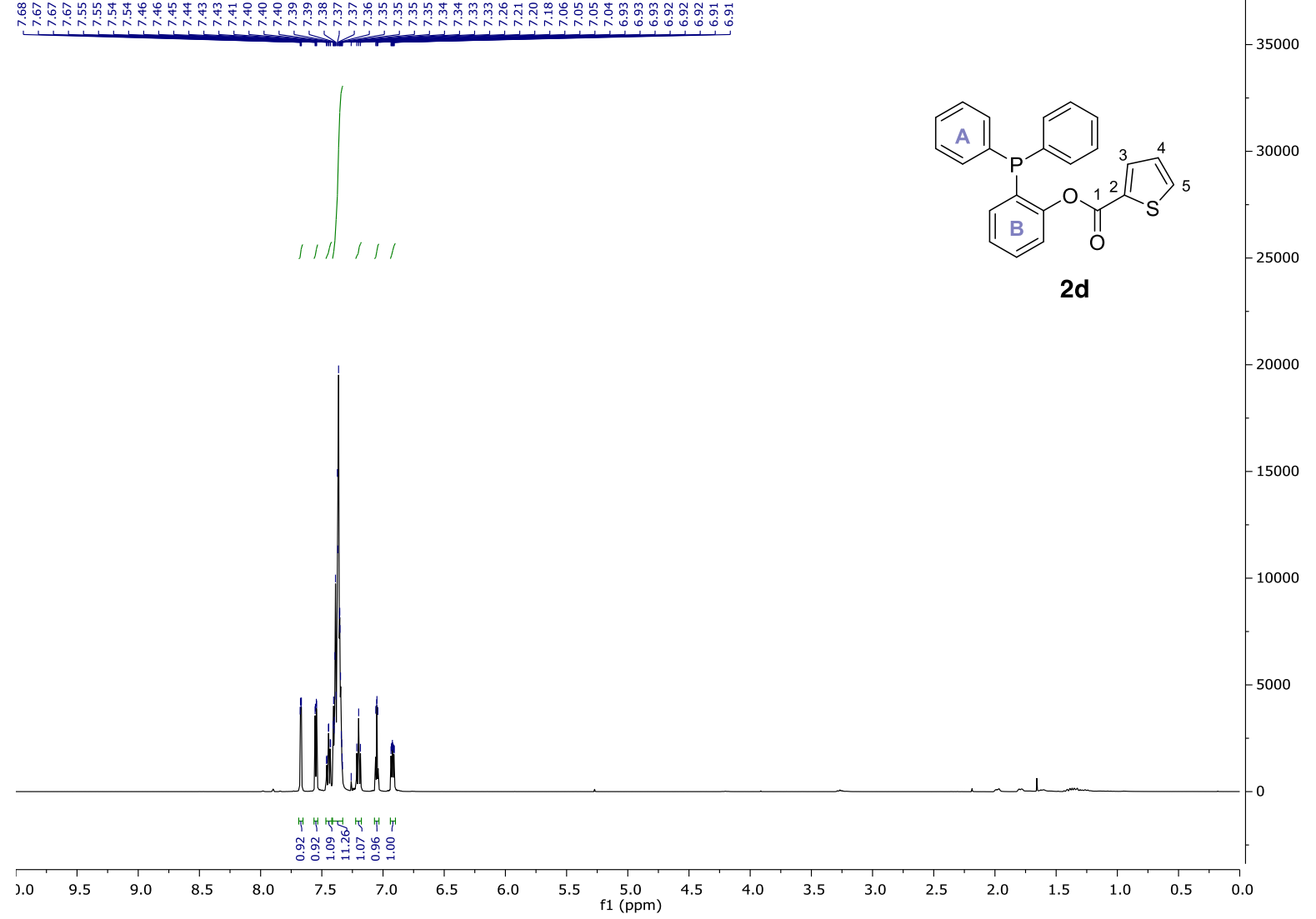


${ }^{13} \mathrm{C} \mathrm{NMR}\left(\mathrm{CDCl}_{3}, 126 \mathrm{MHz}\right)$

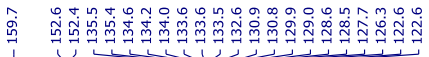

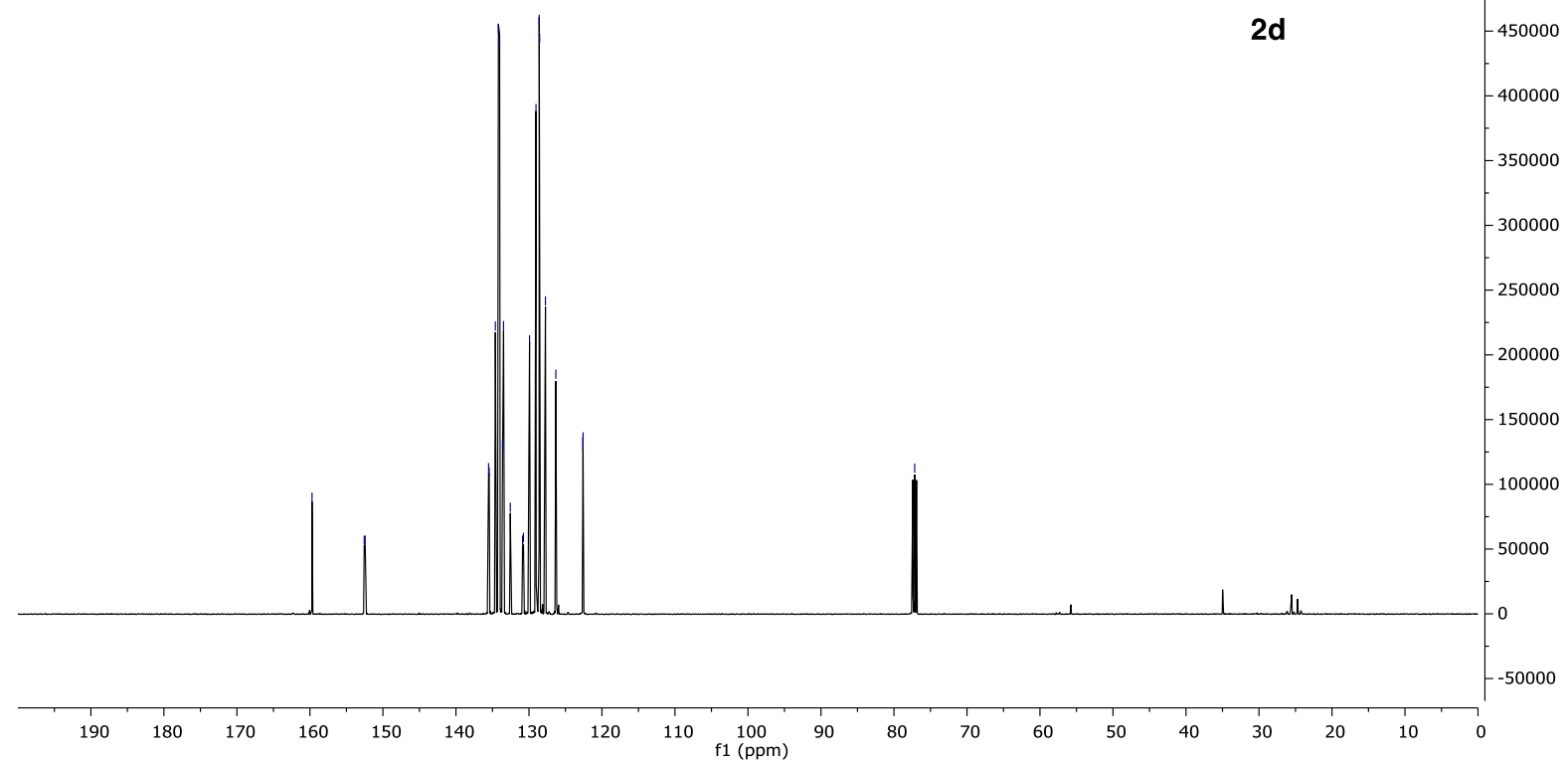

${ }^{31} \mathrm{P} \mathrm{NMR}\left(\mathrm{CDCl}_{3}, 202 \mathrm{MHz}\right)$

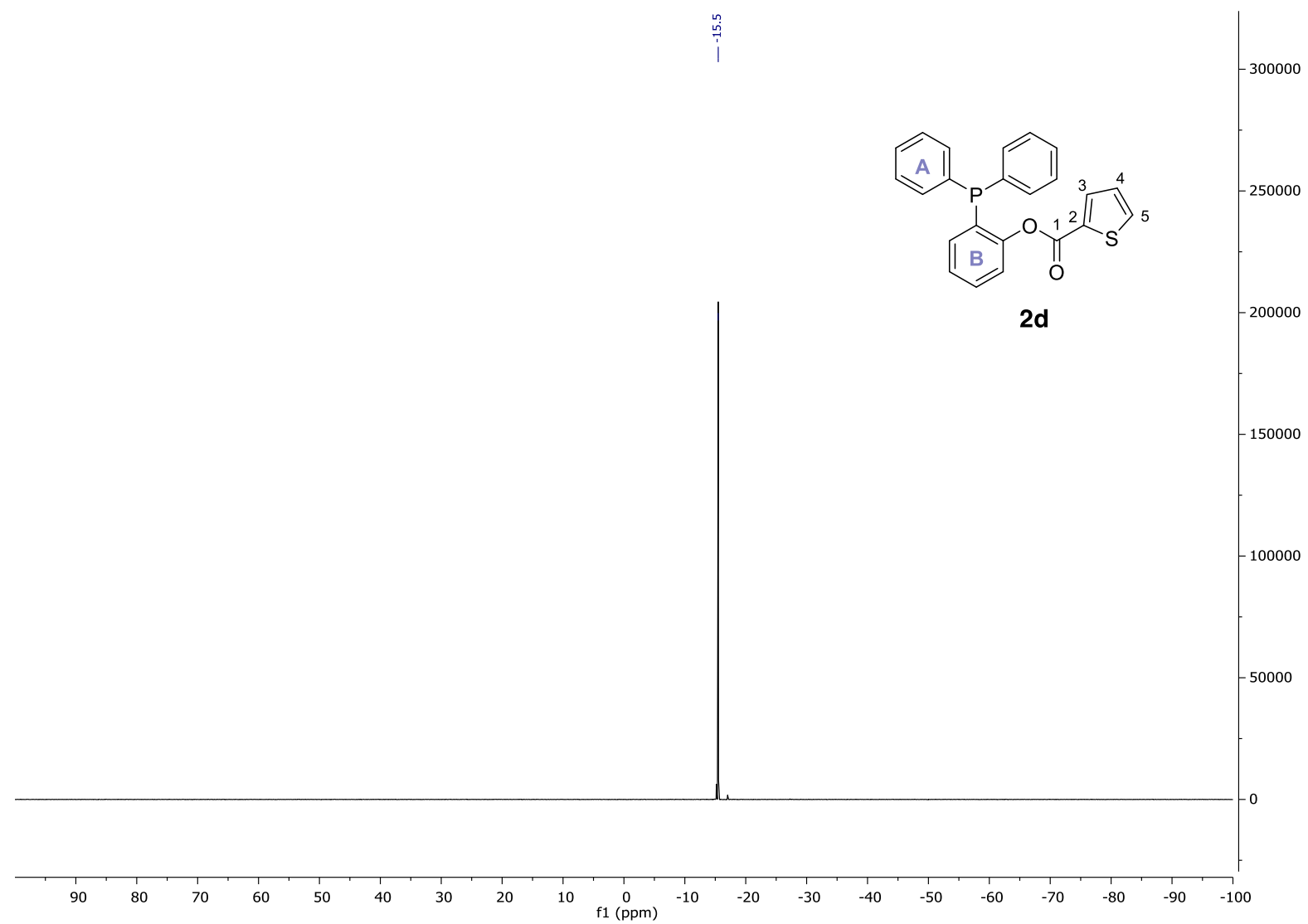


$\left.{ }^{1} \mathrm{H} \mathrm{NMR} \mathrm{(CDCl}{ }_{3}, 500 \mathrm{MHz}\right)$

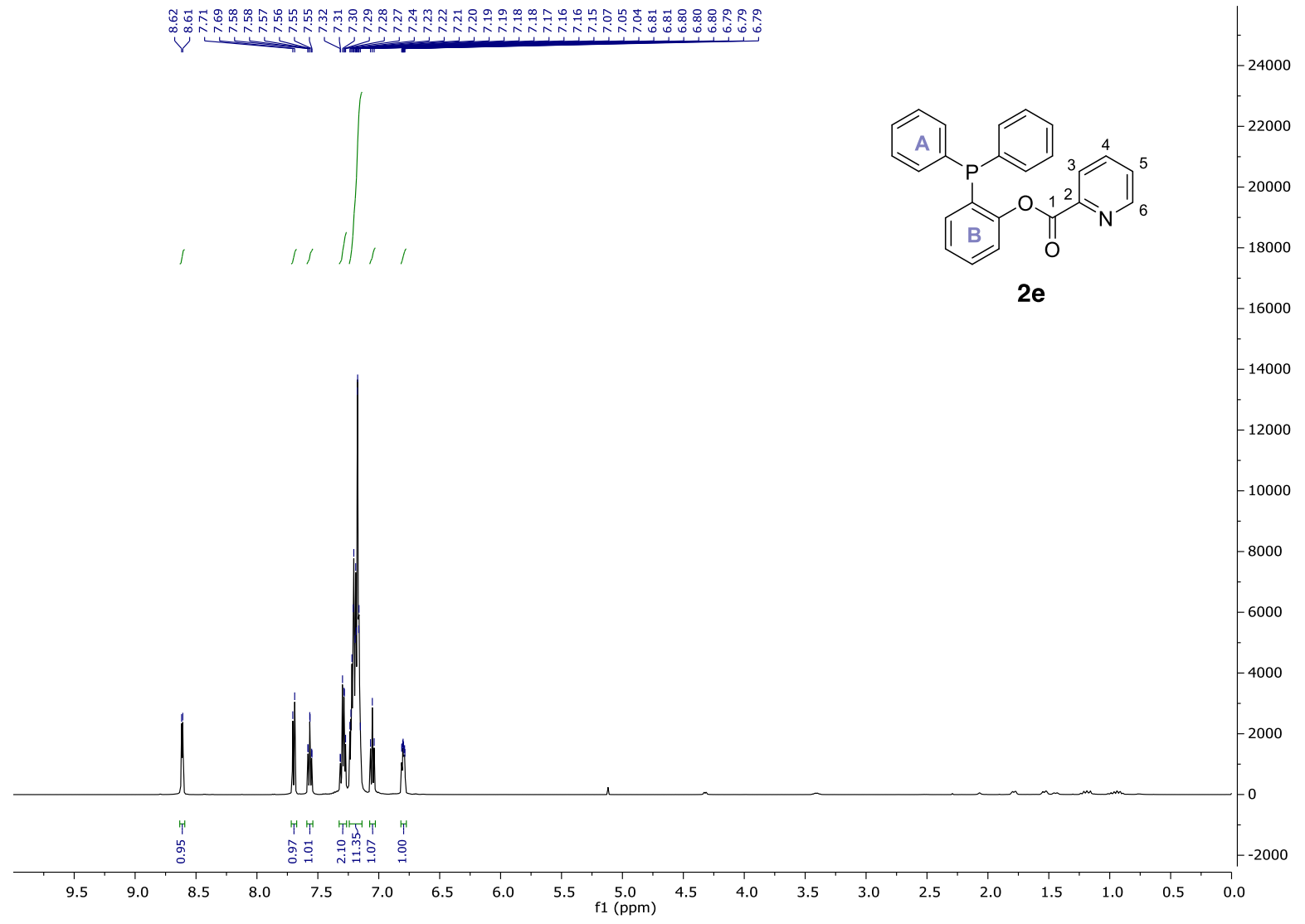

${ }^{13} \mathrm{C} \mathrm{NMR}\left(\mathrm{CDCl}_{3}, 126 \mathrm{MHz}\right)$

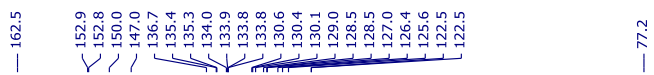
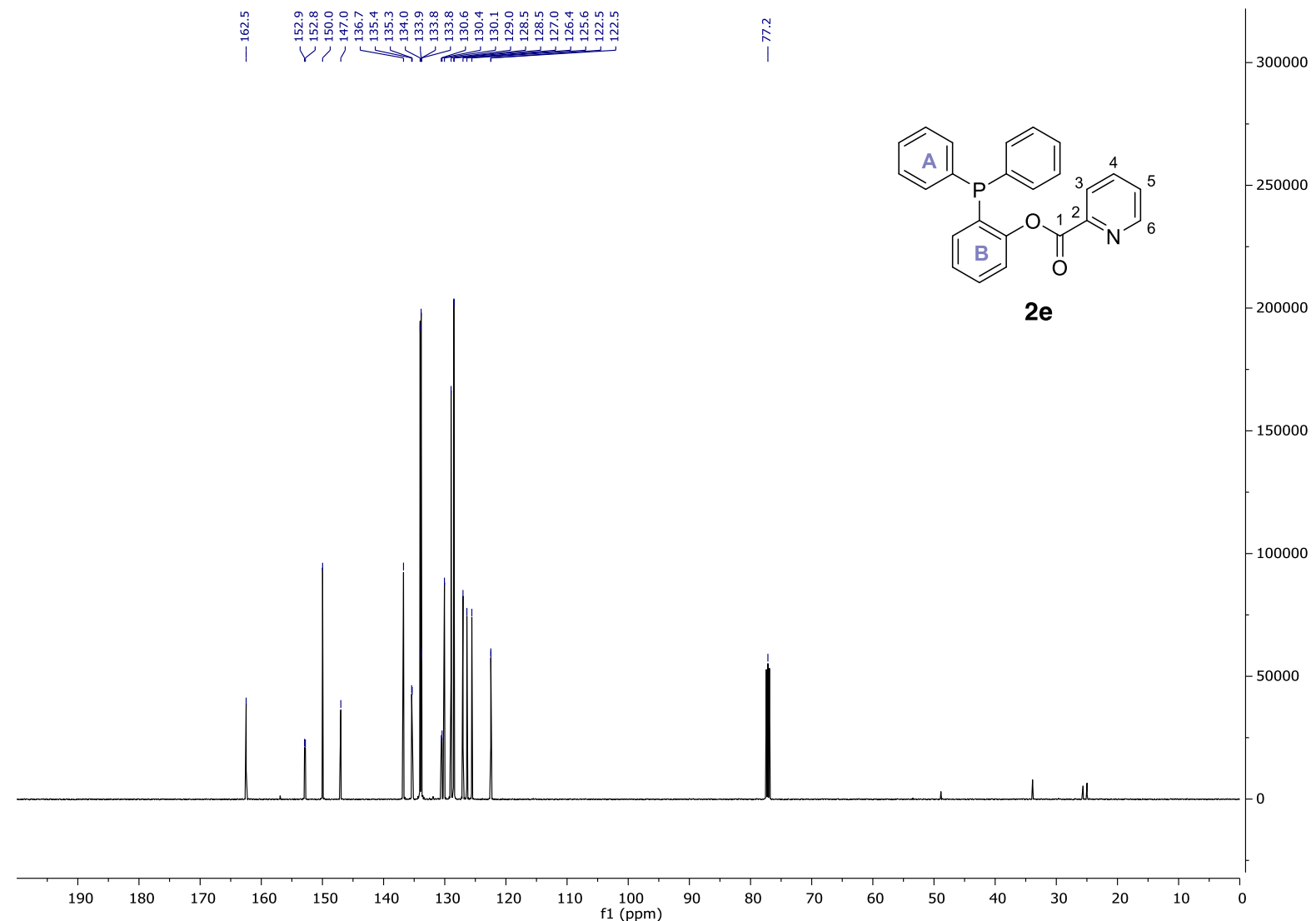
${ }^{31} \mathrm{P}$ NMR $\left(\mathrm{CDCl}_{3}, 202 \mathrm{MHz}\right)$

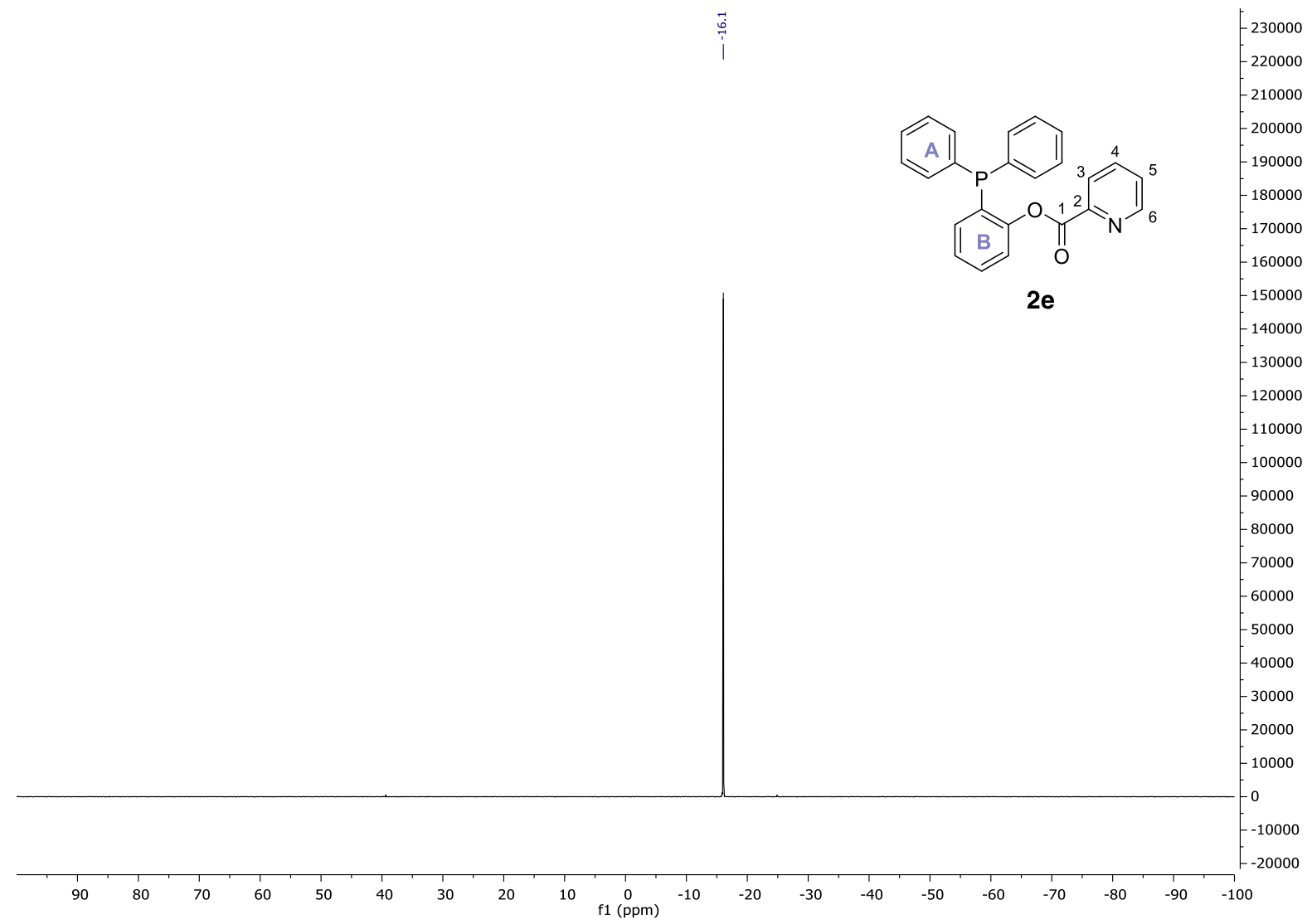

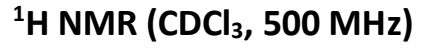

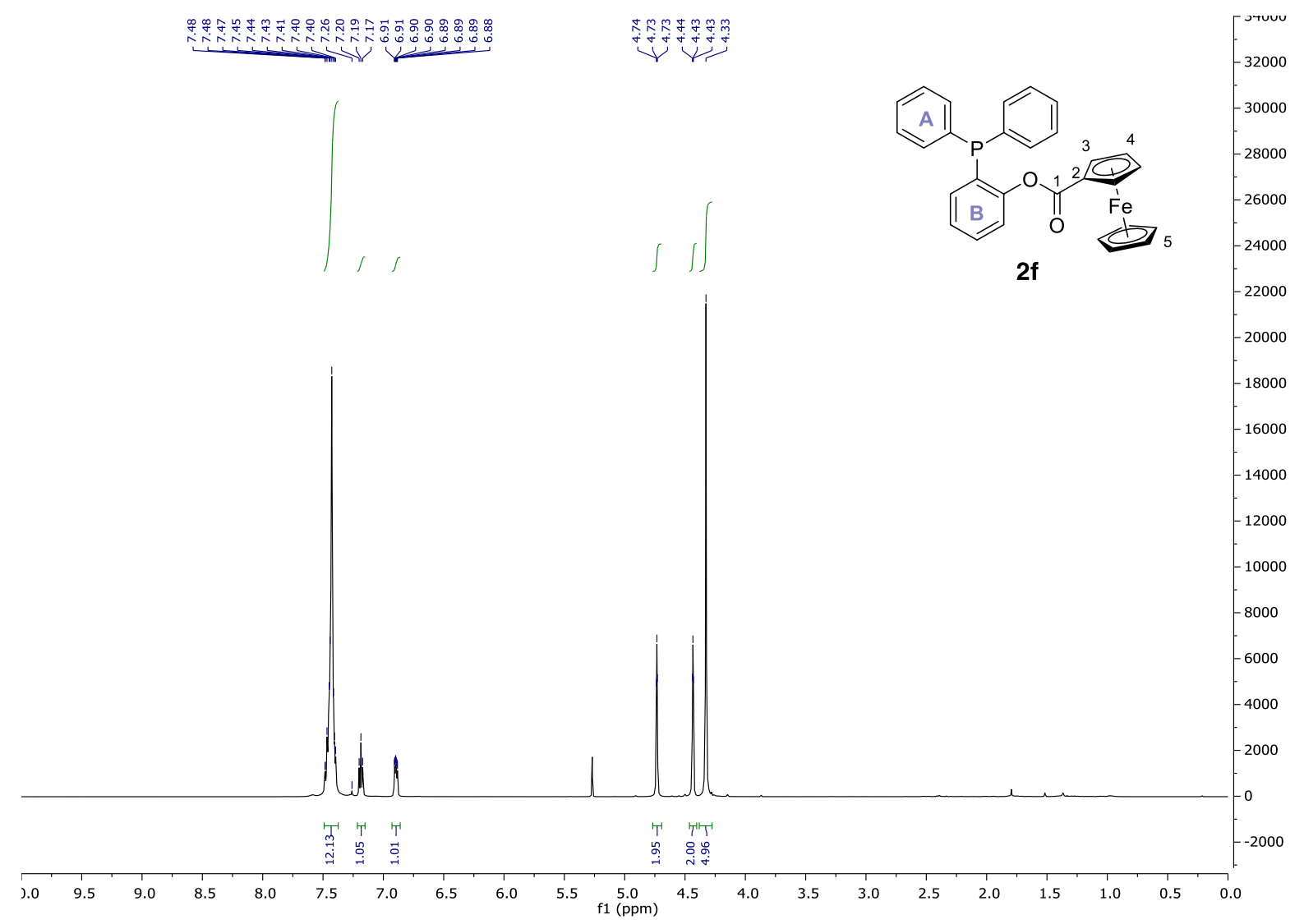


${ }^{13} \mathrm{C} \mathrm{NMR}\left(\mathrm{CDCl}_{3}, 126 \mathrm{MHz}\right)$

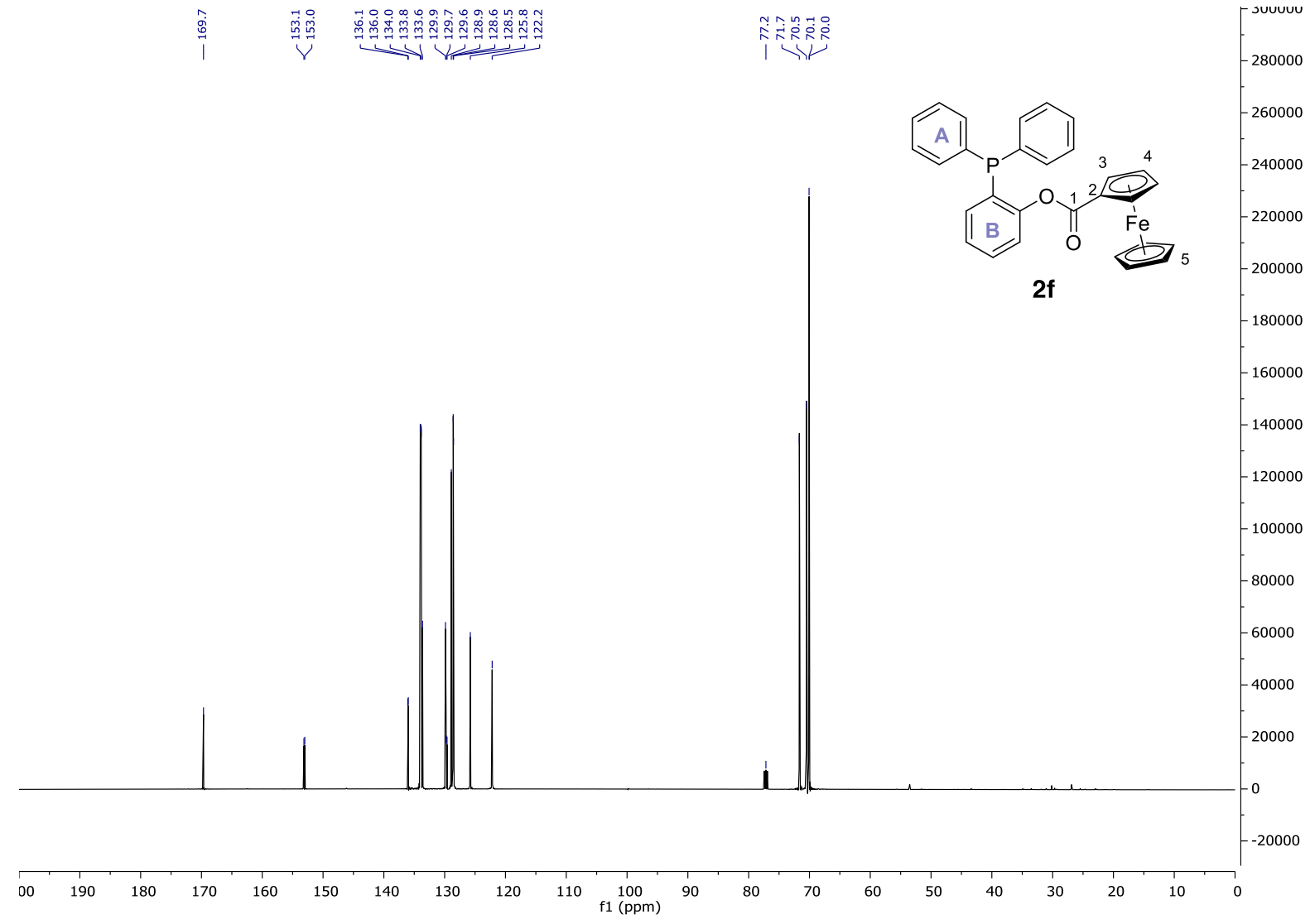

${ }^{31} \mathrm{P} \mathrm{NMR}\left(\mathrm{CDCl}_{3}, 202 \mathrm{MHz}\right)$

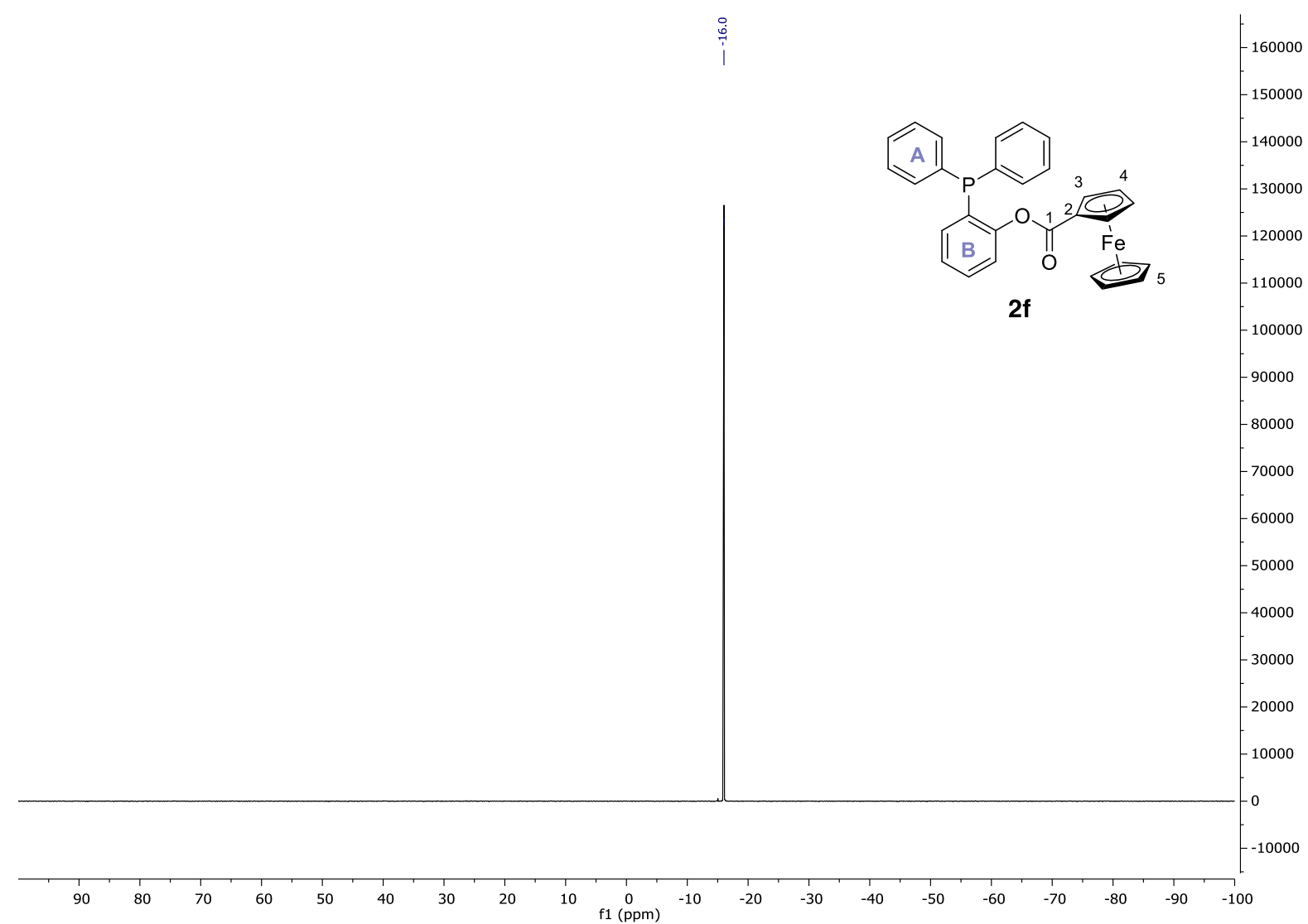


${ }^{1} \mathrm{H}$ NMR $\left(\mathrm{CDCl}_{3}, 500 \mathrm{MHz}\right)$

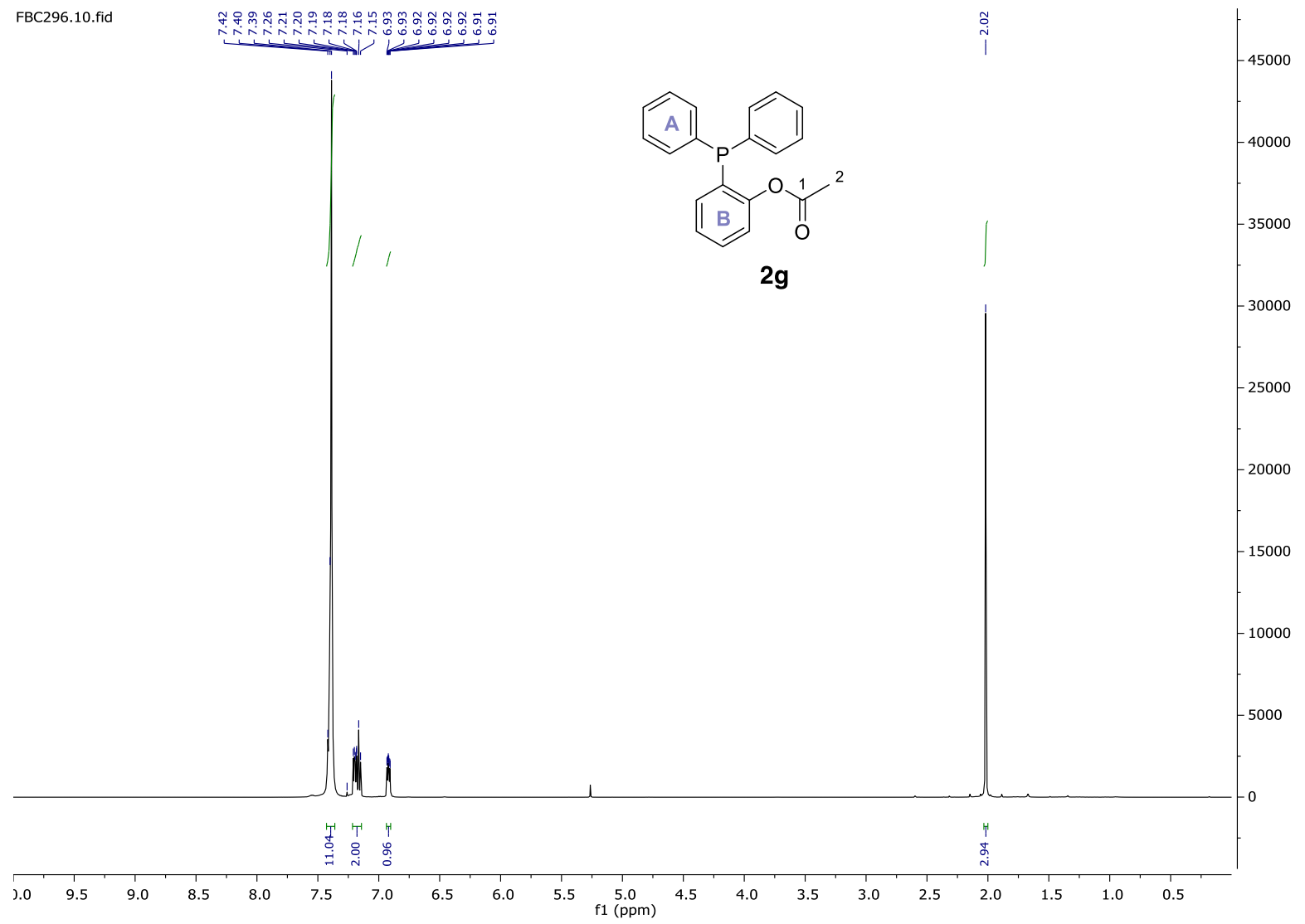

$\left.{ }^{13} \mathrm{C} \mathrm{NMR} \mathrm{(CDCl}, 126 \mathrm{MHz}\right)$

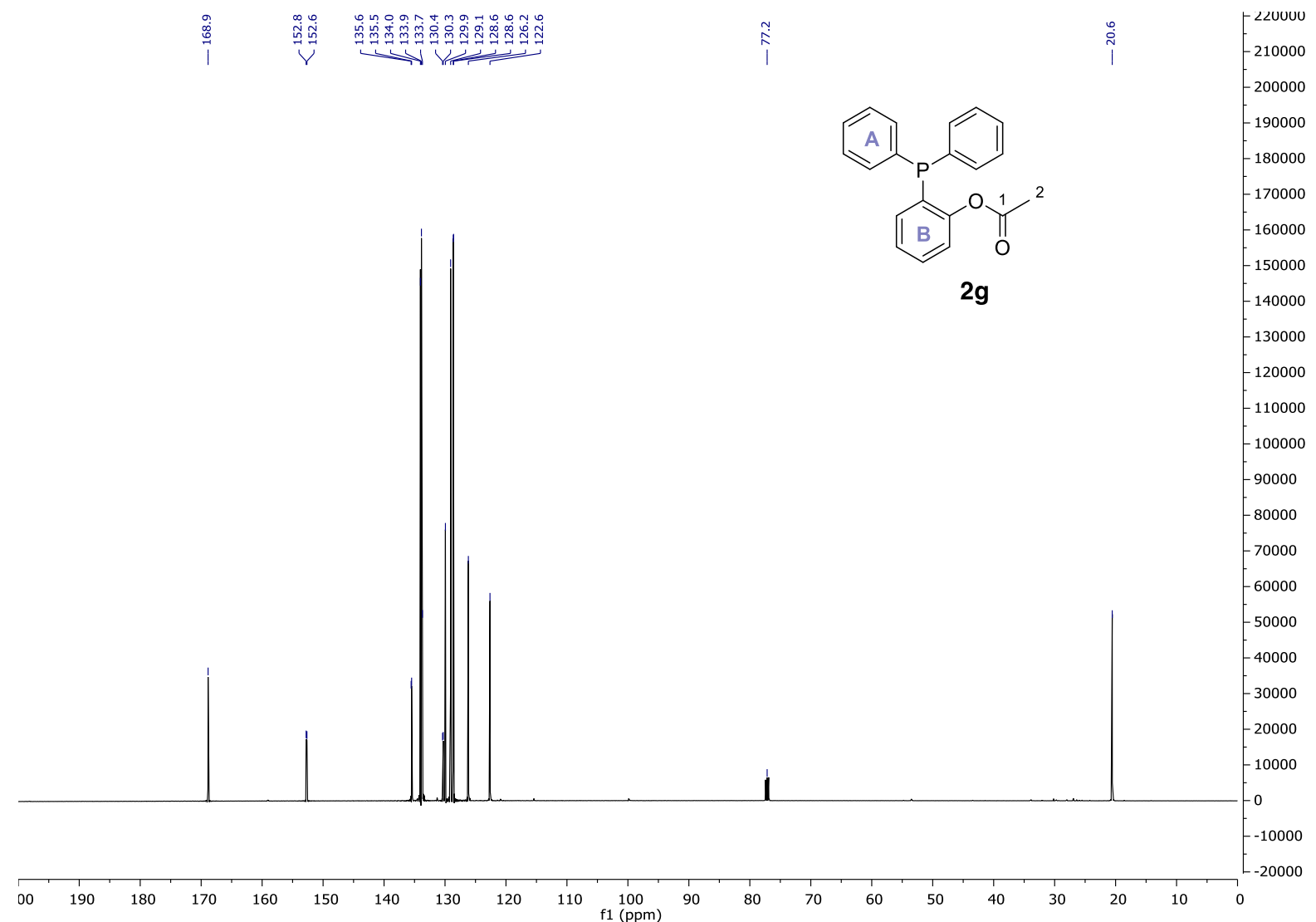


${ }^{31} \mathrm{P}$ NMR $\left(\mathrm{CDCl}_{3}, 202 \mathrm{MHz}\right)$

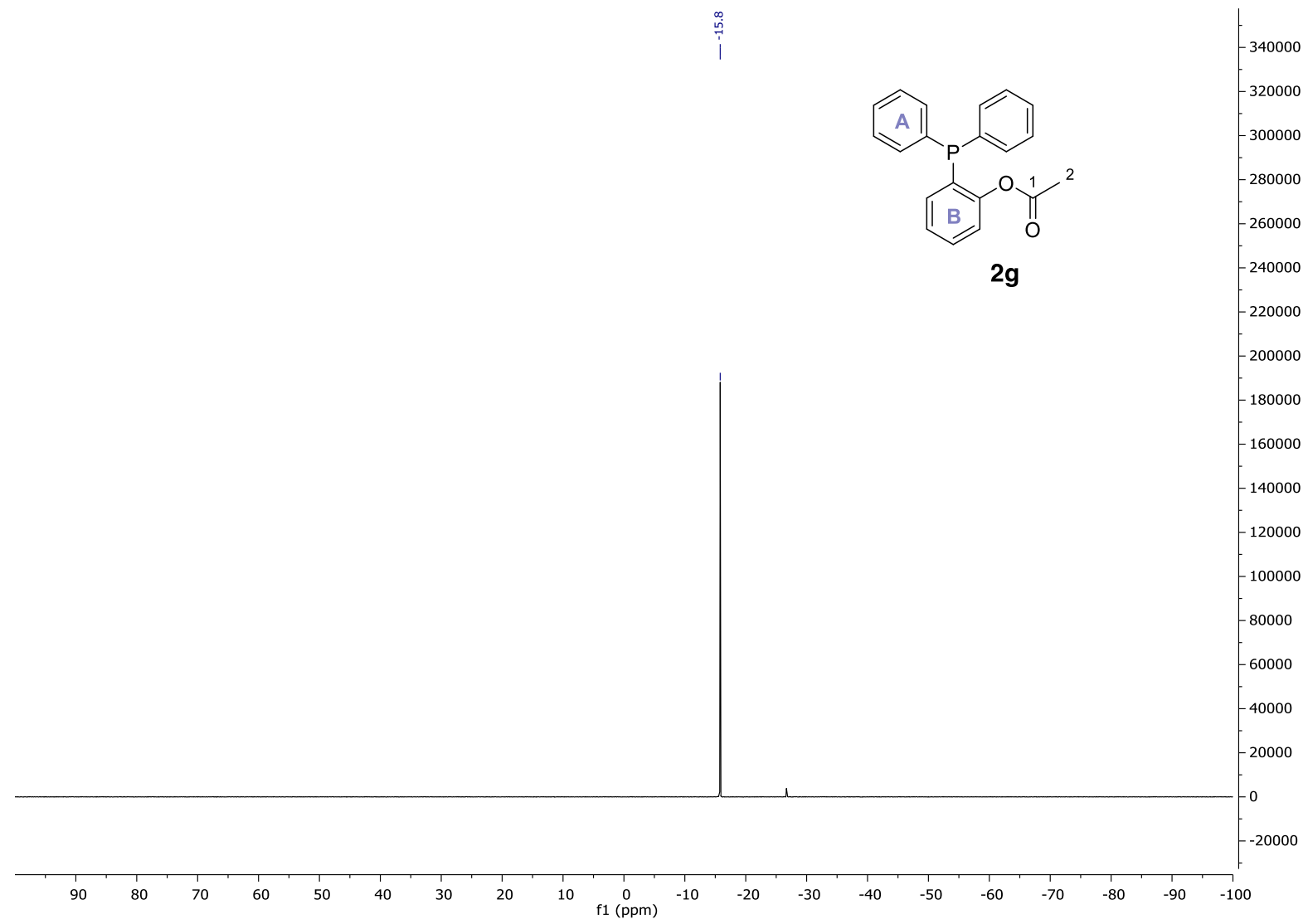

${ }^{1} \mathrm{H} \mathrm{NMR}\left(\mathrm{CDCl}_{3}, 500 \mathrm{MHz}\right)$

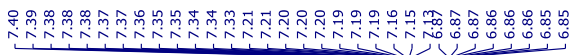

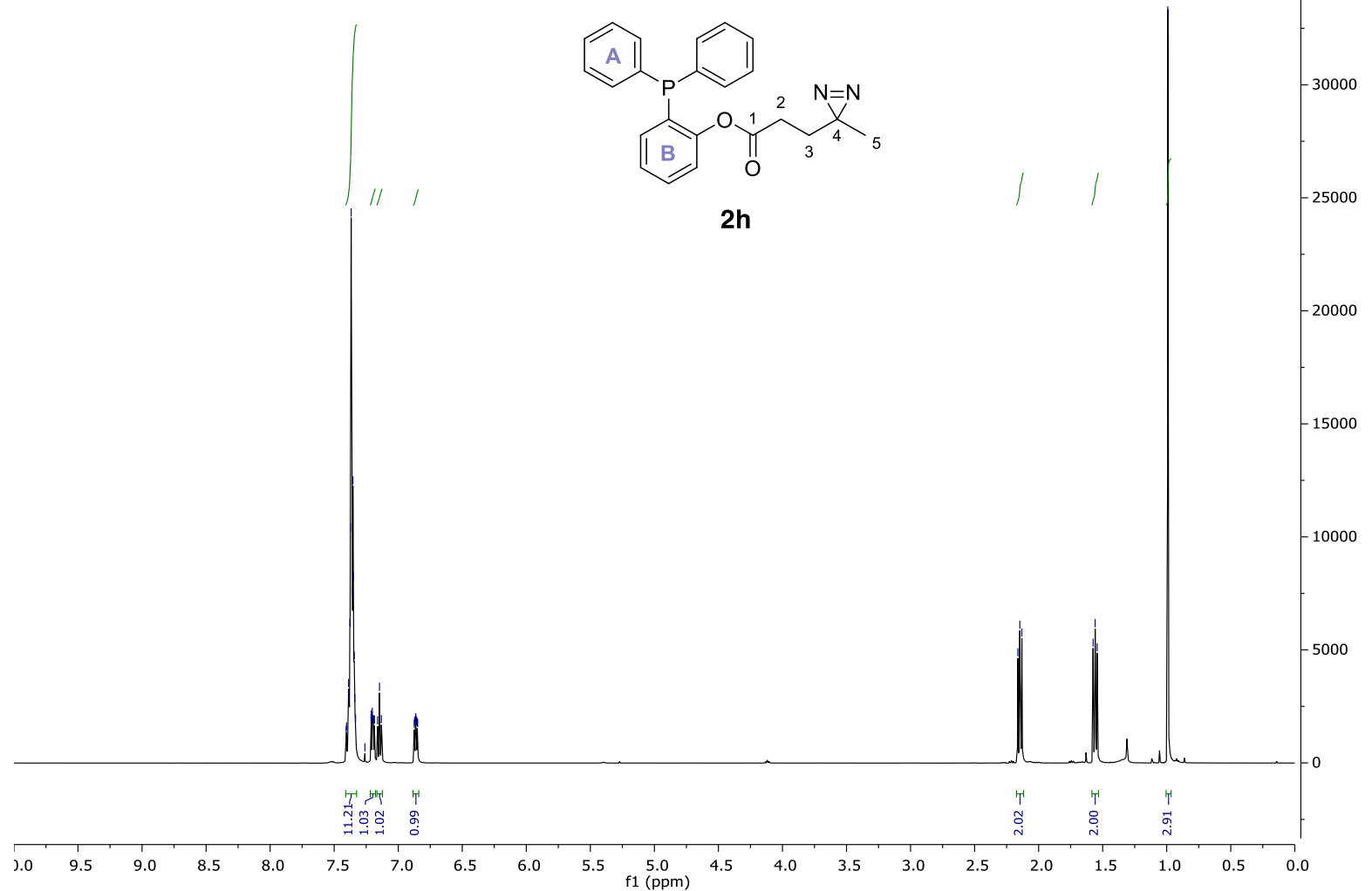


${ }^{13} \mathrm{C} \mathrm{NMR}\left(\mathrm{CDCl}_{3}, 126 \mathrm{MHz}\right)$

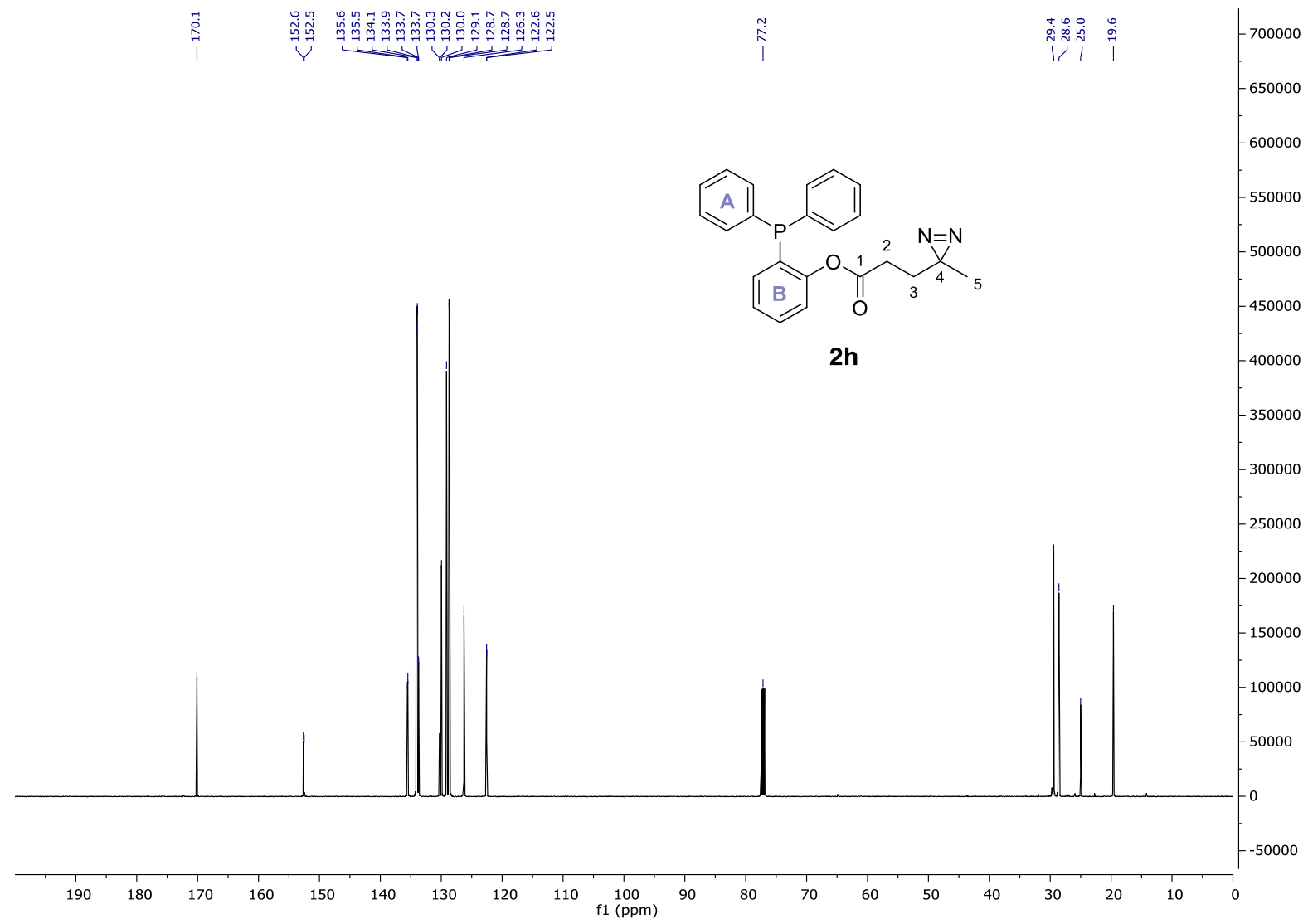

${ }^{31} \mathrm{P} \mathrm{NMR}\left(\mathrm{CDCl}_{3}, 202 \mathrm{MHz}\right)$

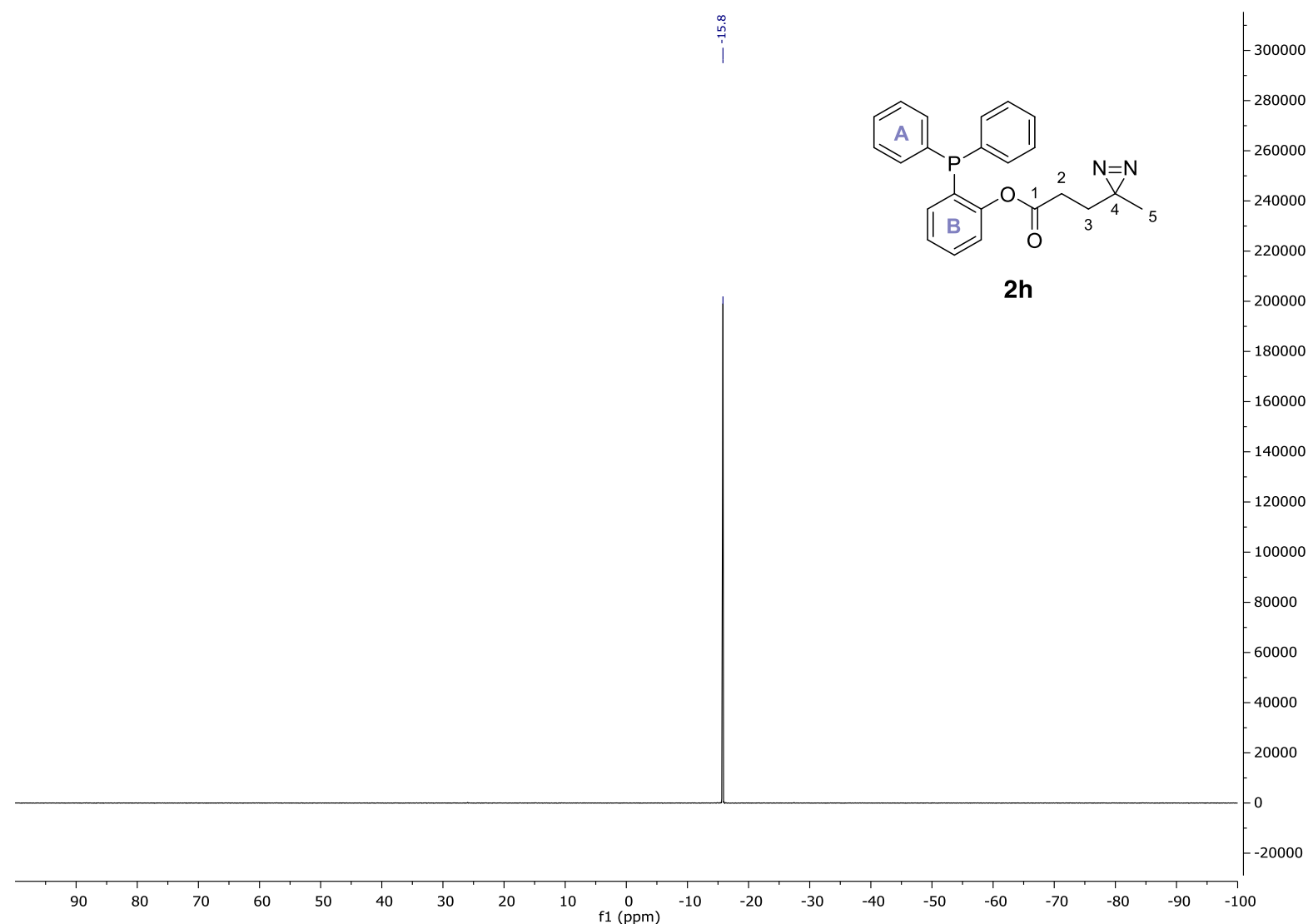


${ }^{1} \mathrm{H}$ NMR $\left(\mathrm{CDCl}_{3}, 500 \mathrm{MHz}\right)$

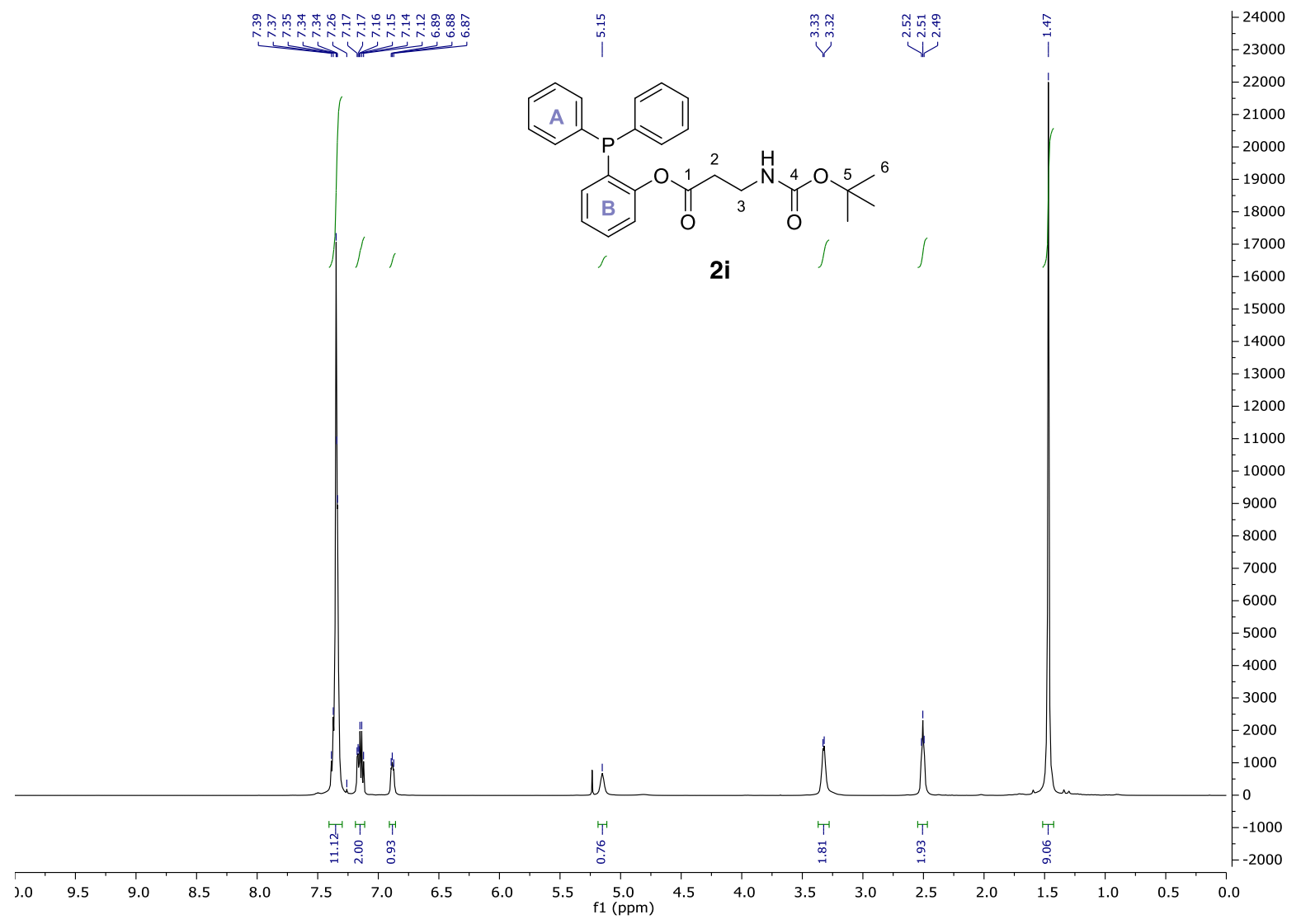

$\left.{ }^{13} \mathrm{C} \mathrm{NMR} \mathrm{(CDCl} 3,126 \mathrm{MHz}\right)$

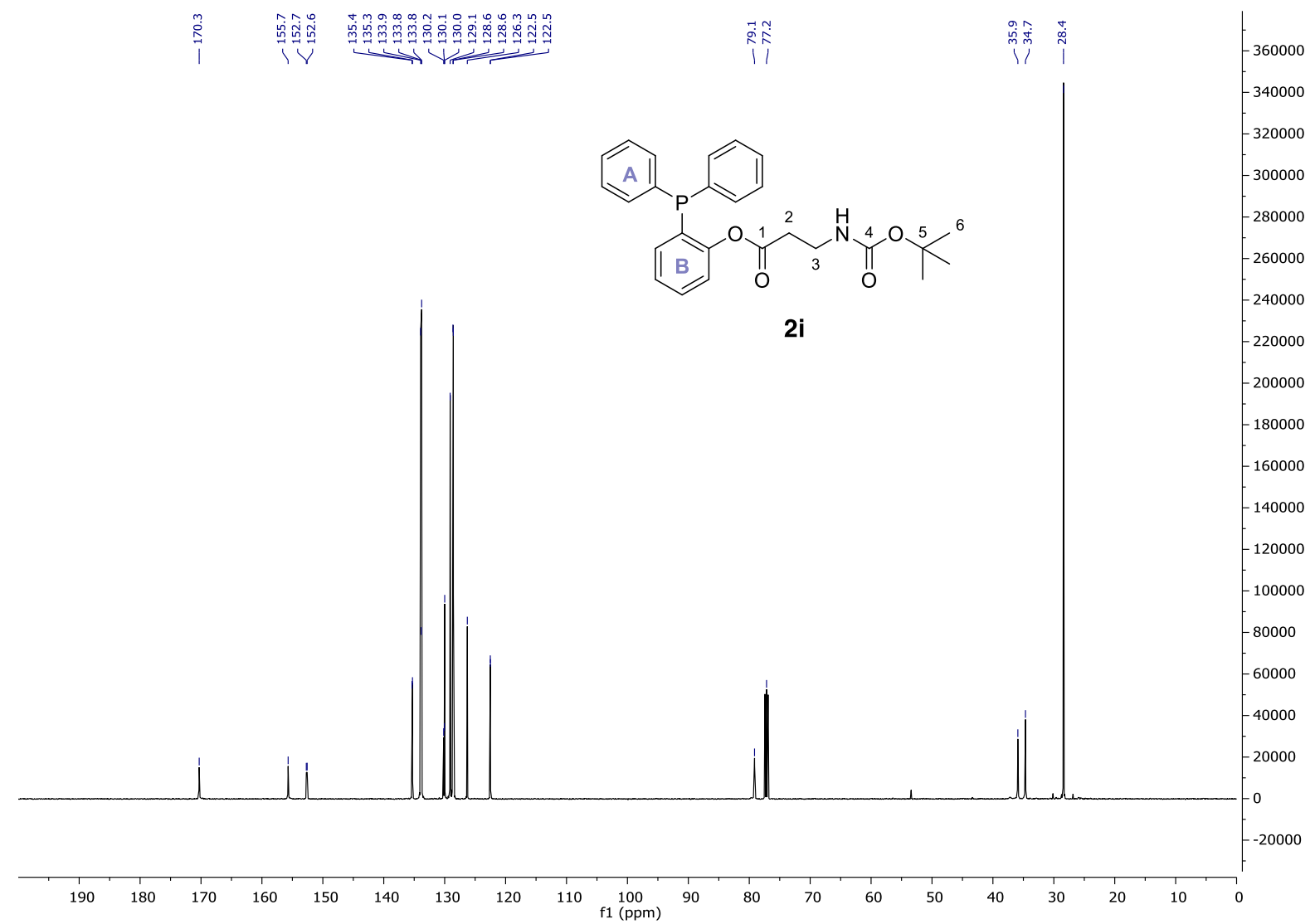


${ }^{31} \mathrm{P}$ NMR $\left(\mathrm{CDCl}_{3}, 202 \mathrm{MHz}\right)$

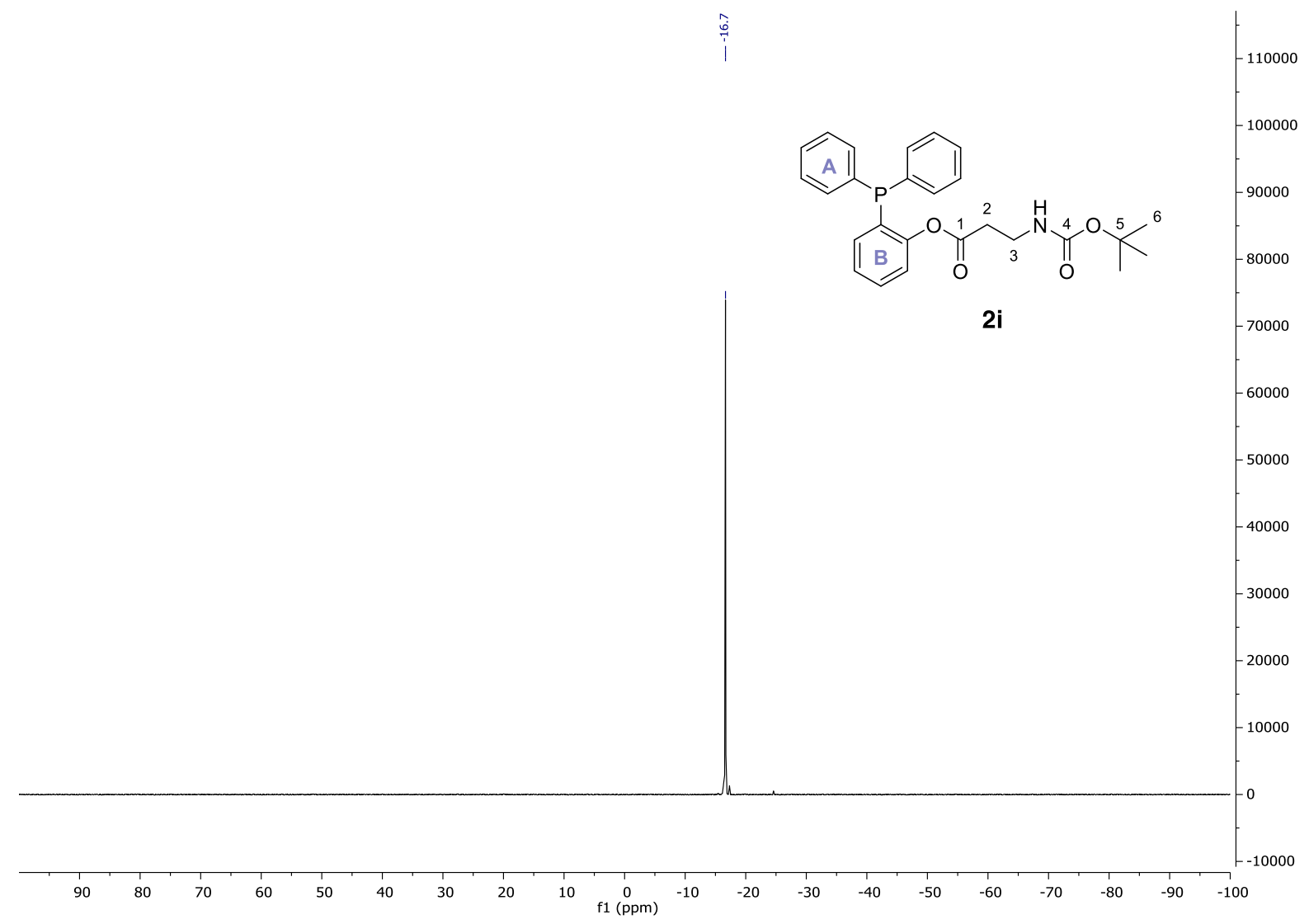

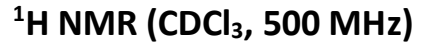

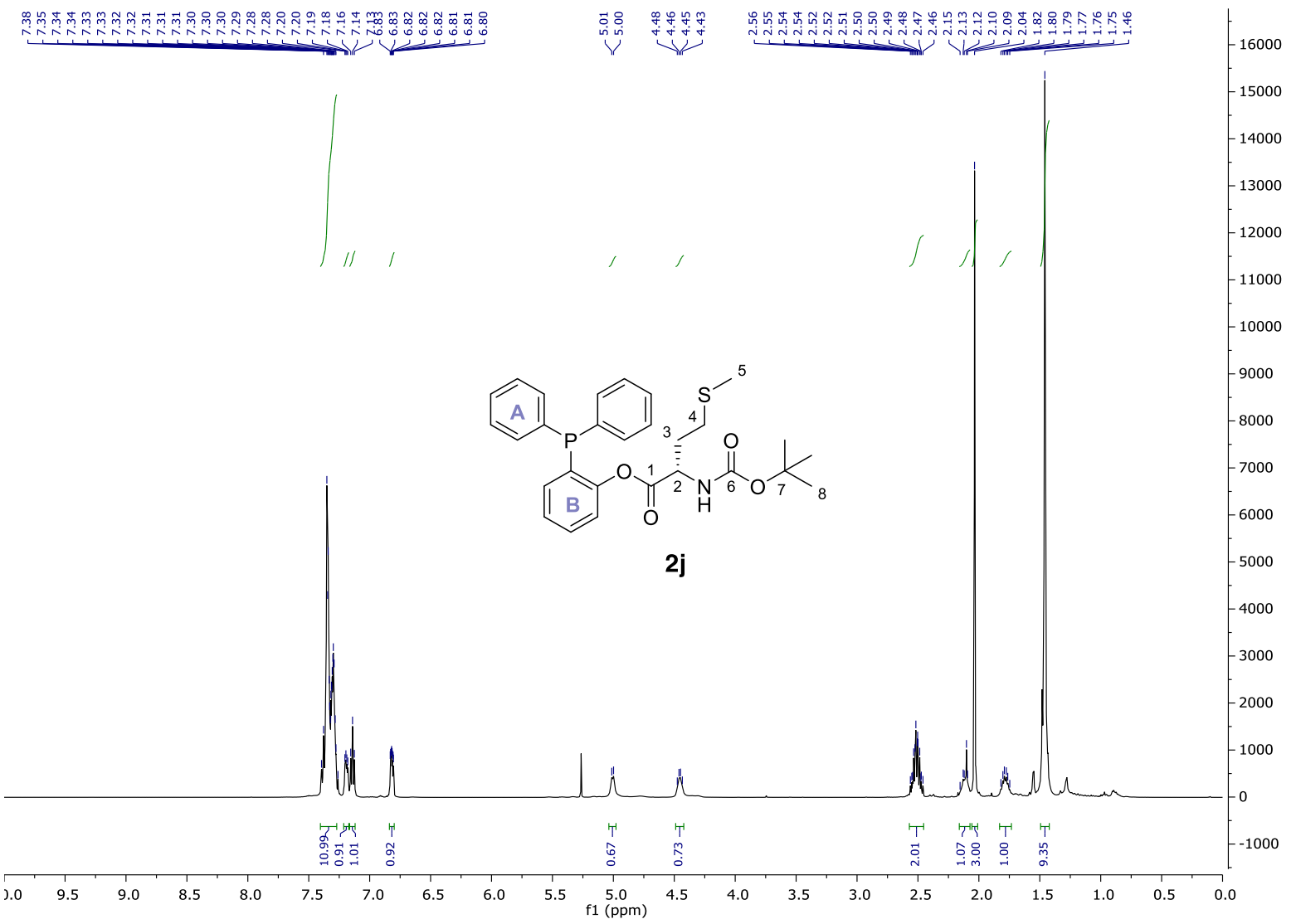


${ }^{13} \mathrm{C} \mathrm{NMR}\left(\mathrm{CDCl}_{3}, 126 \mathrm{MHz}\right)$

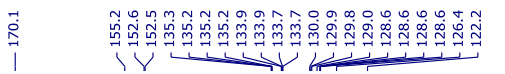

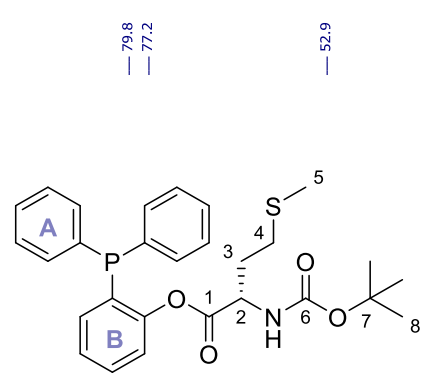

2j

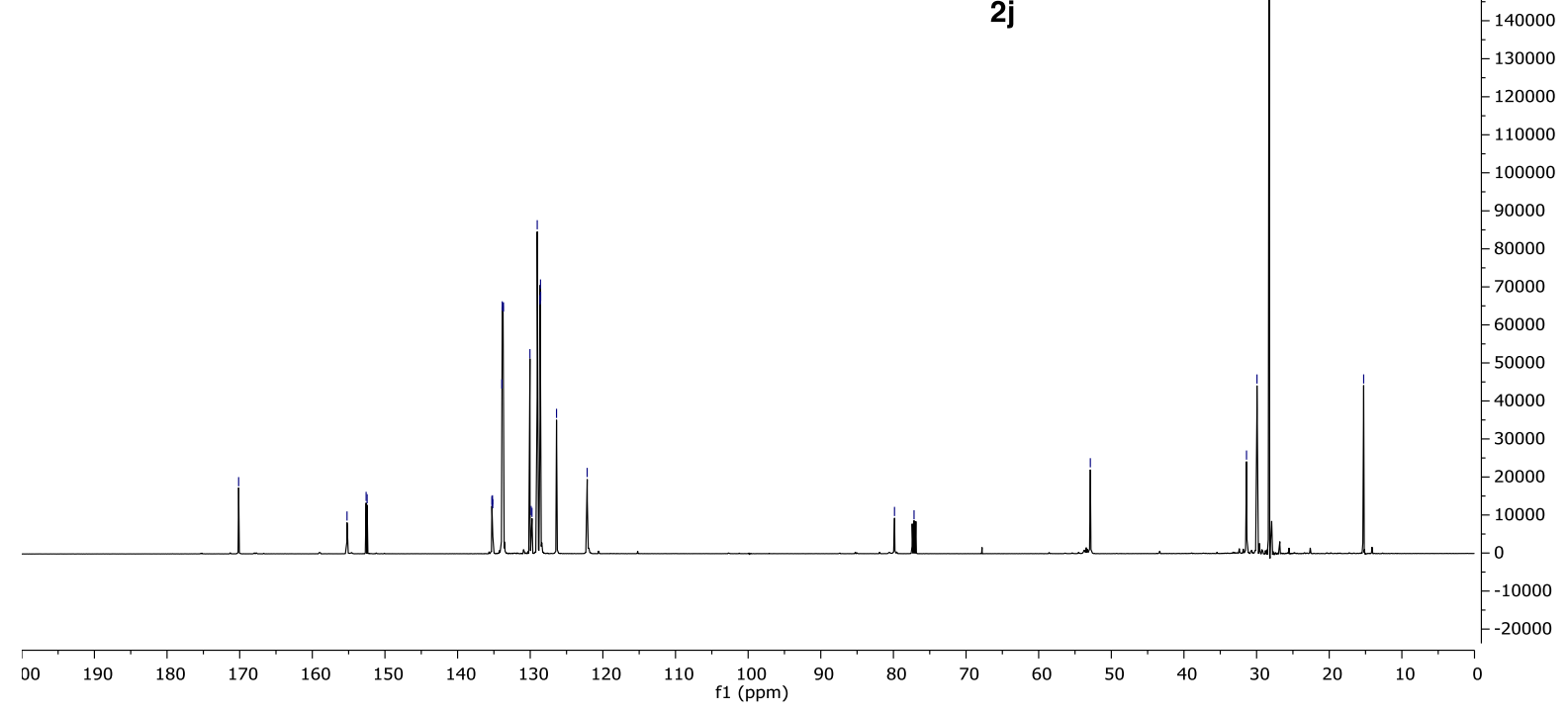

${ }^{31} \mathrm{P} \mathrm{NMR}\left(\mathrm{CDCl}_{3}, 202 \mathrm{MHz}\right)$

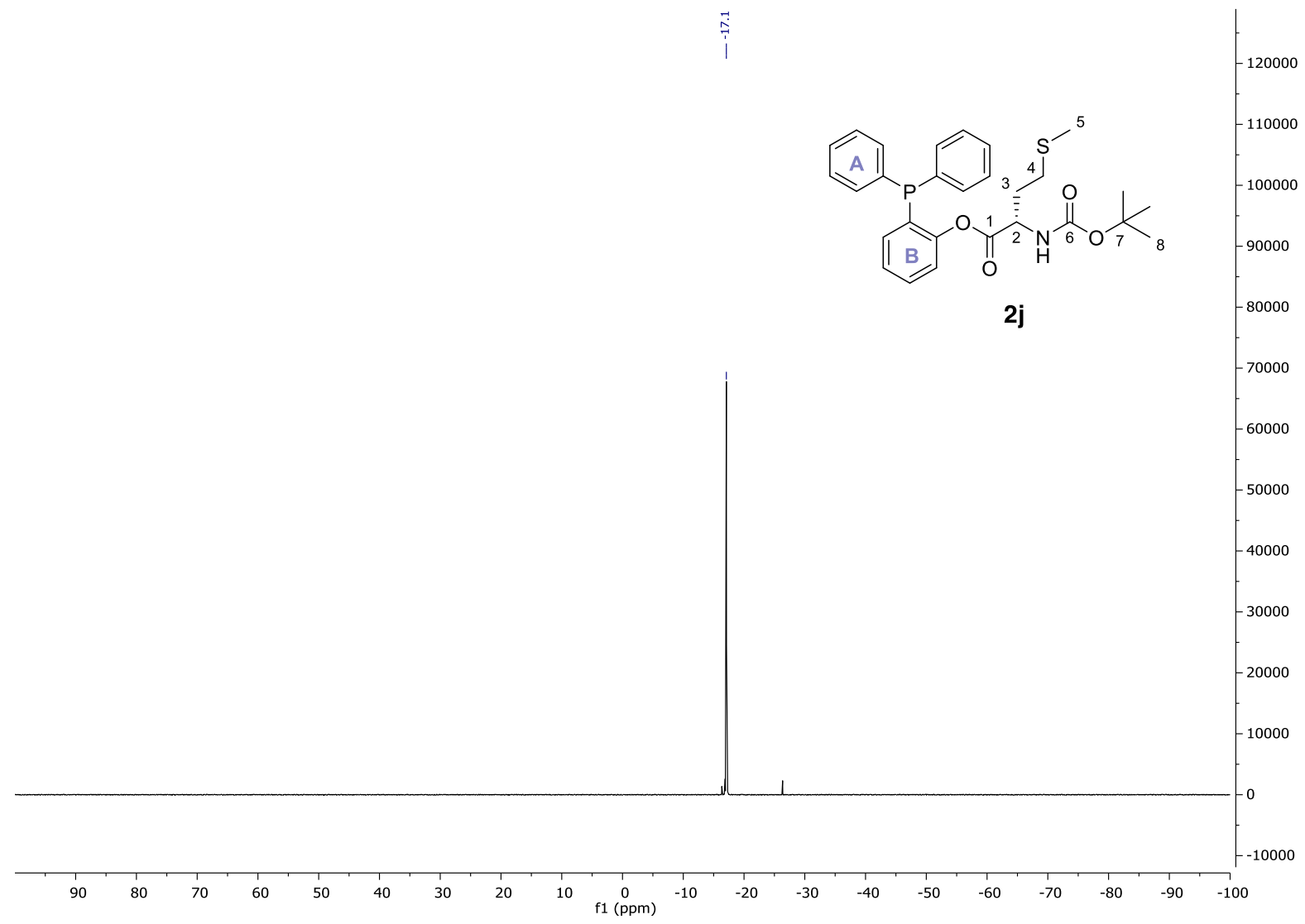


${ }^{1} \mathrm{H}$ NMR $\left(\mathrm{CDCl}_{3}, 500 \mathrm{MHz}\right)$

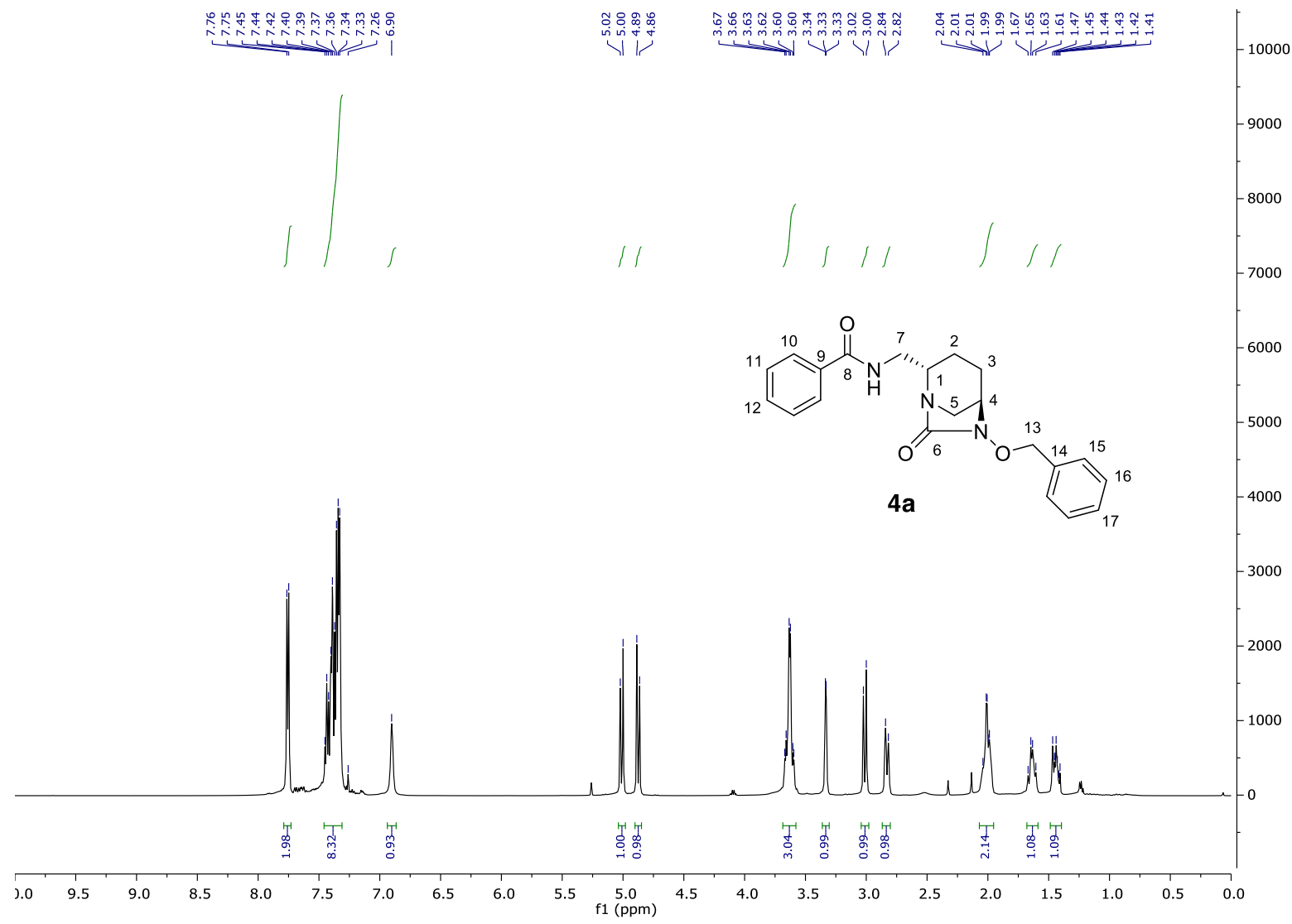

$\left.{ }^{13} \mathrm{C} \mathrm{NMR} \mathrm{(CDCl} 3,126 \mathrm{MHz}\right)$

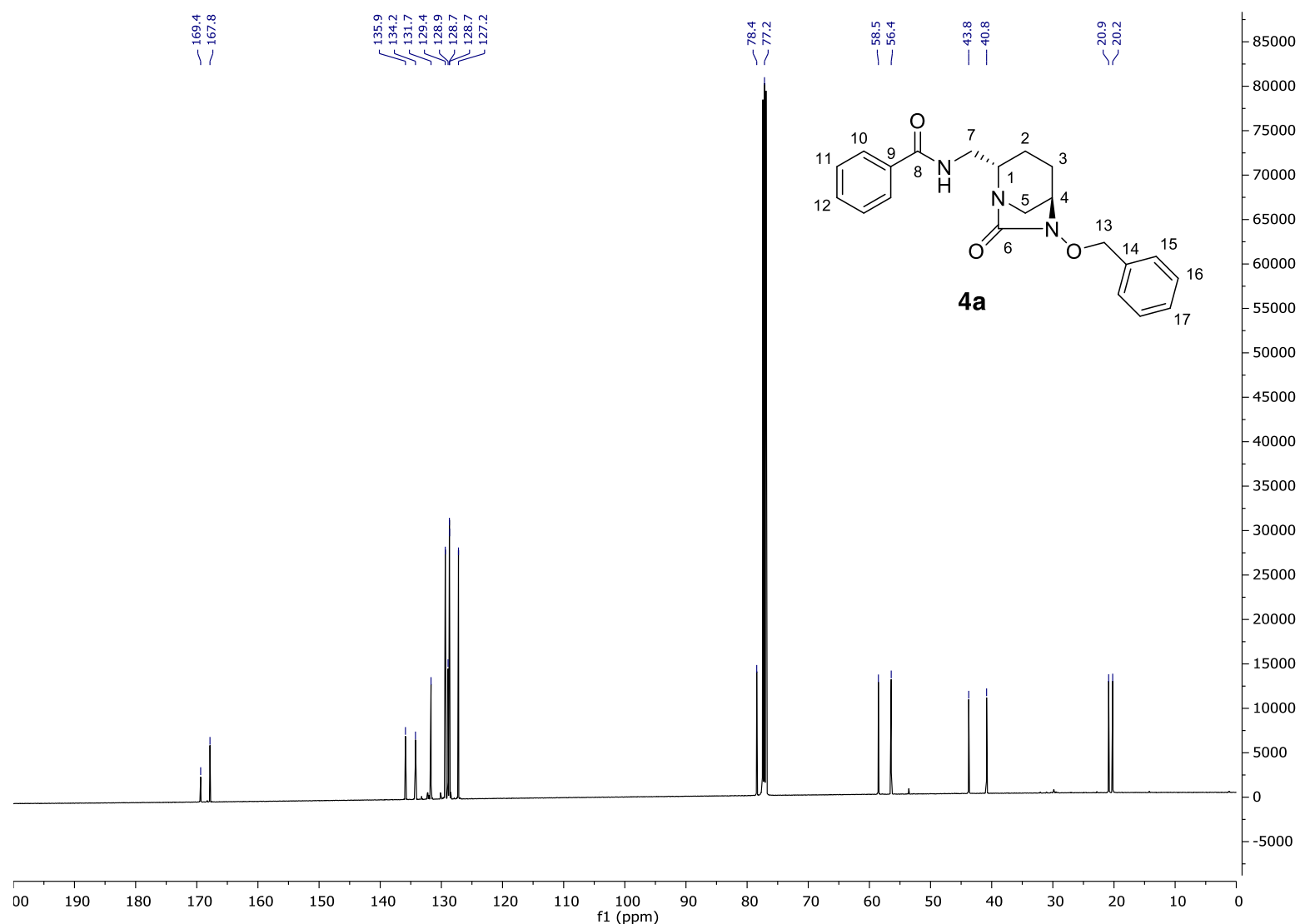


${ }^{1} \mathrm{H}$ NMR $\left(\mathrm{CDCl}_{3}, 500 \mathrm{MHz}\right)$

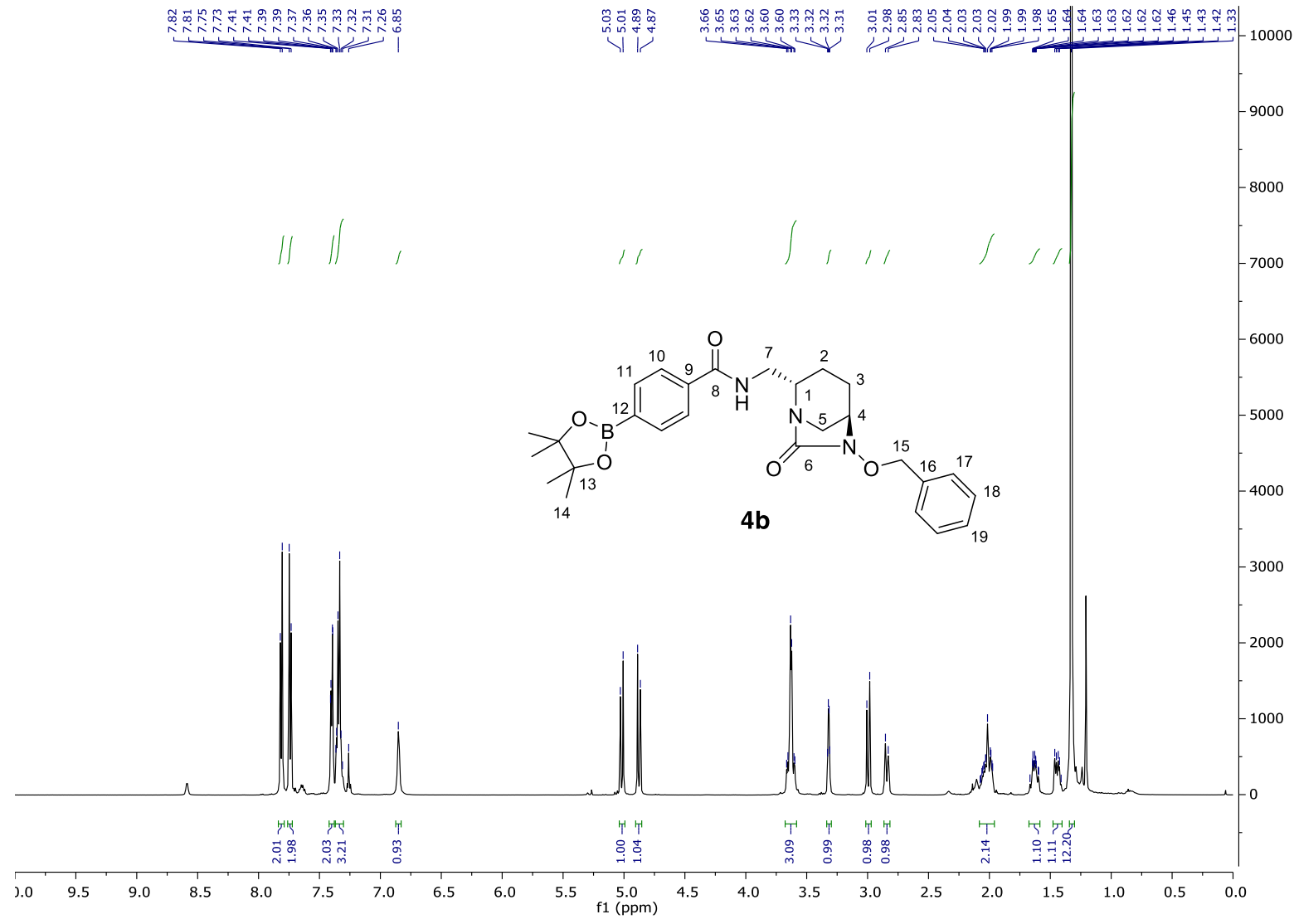

${ }^{13} \mathrm{C}$ NMR $\left(\mathrm{CDCl}_{3}, 126 \mathrm{MHz}\right)$

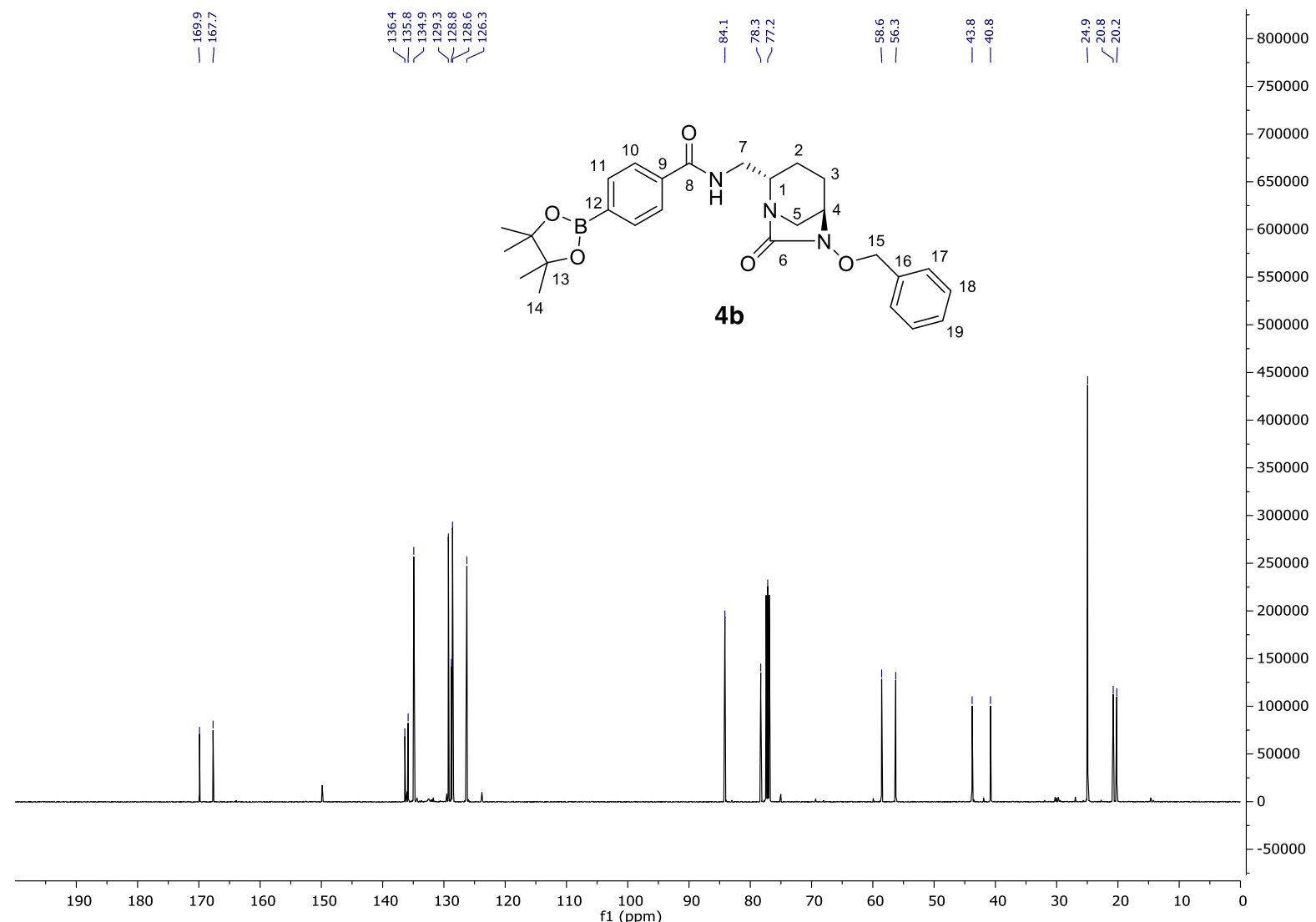


${ }^{1} \mathrm{H} \mathrm{NMR}\left(\mathrm{CDCl}_{3}, 500 \mathrm{MHz}\right)$

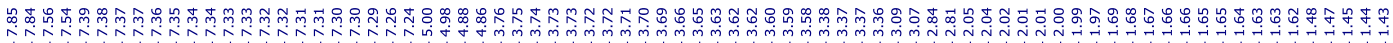

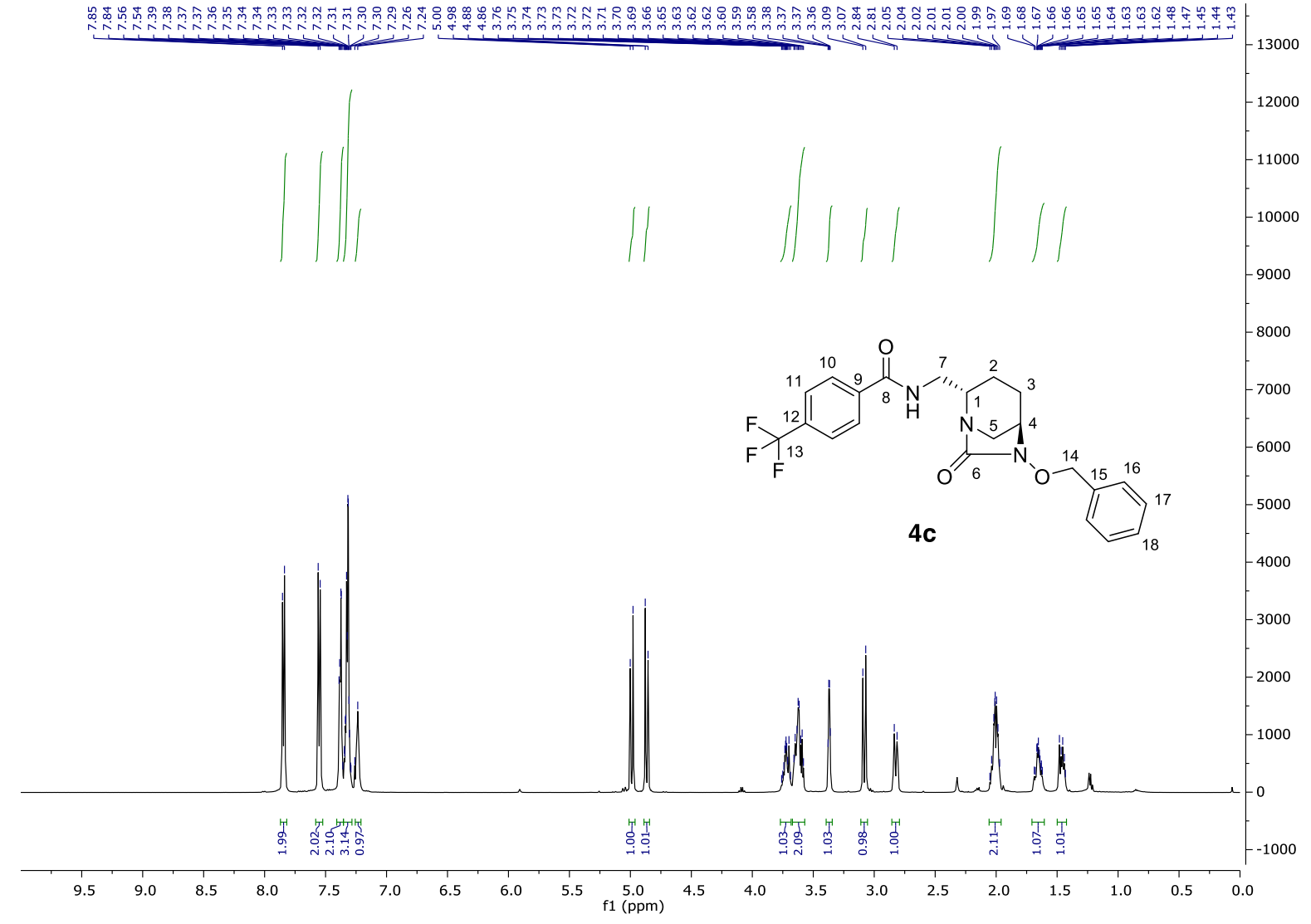

$\left.{ }^{13} \mathrm{C} \mathrm{NMR} \mathrm{(CDCl}, 126 \mathrm{MHz}\right)$

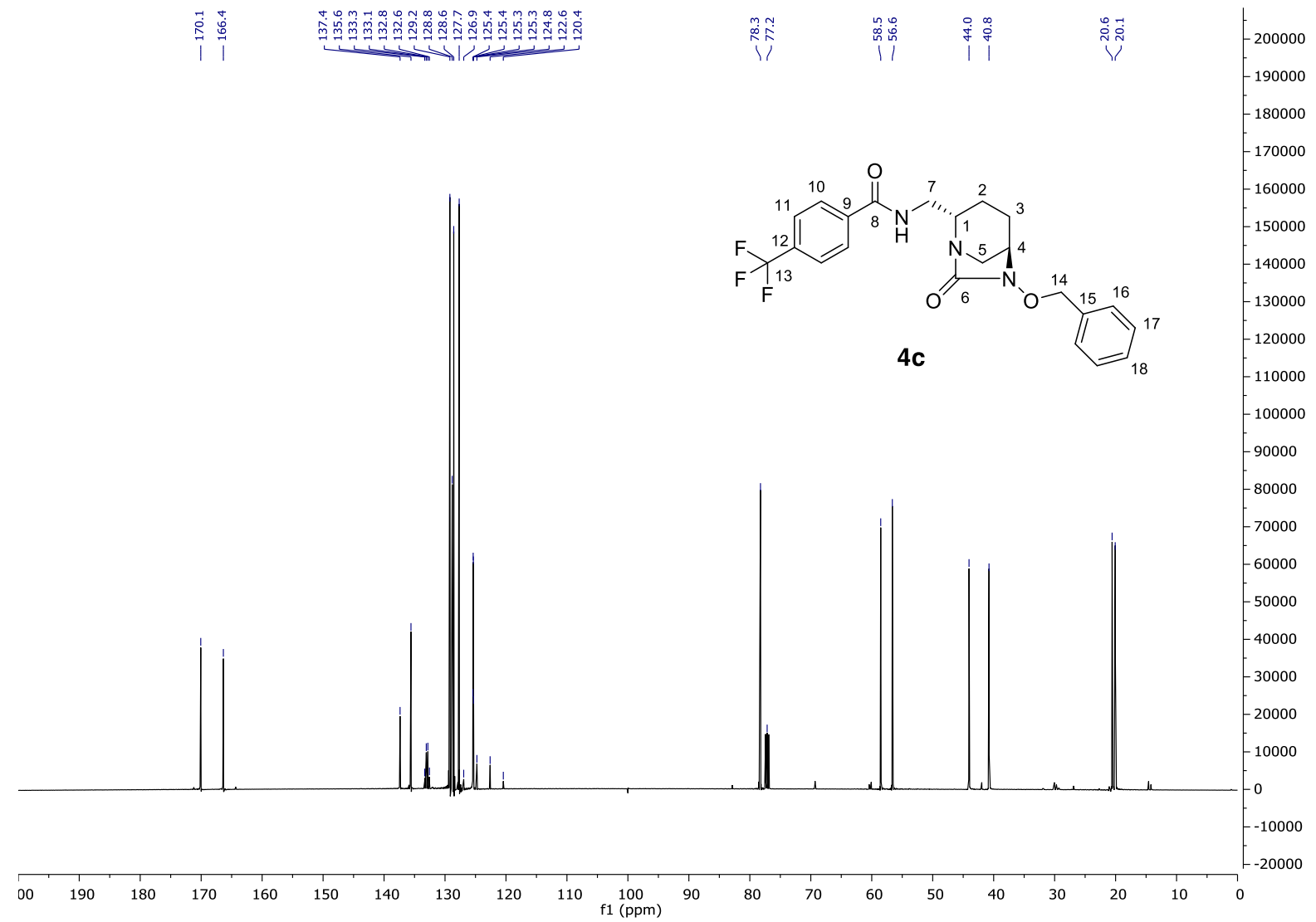


${ }^{1} \mathrm{H}$ NMR $\left(\mathrm{CDCl}_{3}, 500 \mathrm{MHz}\right)$

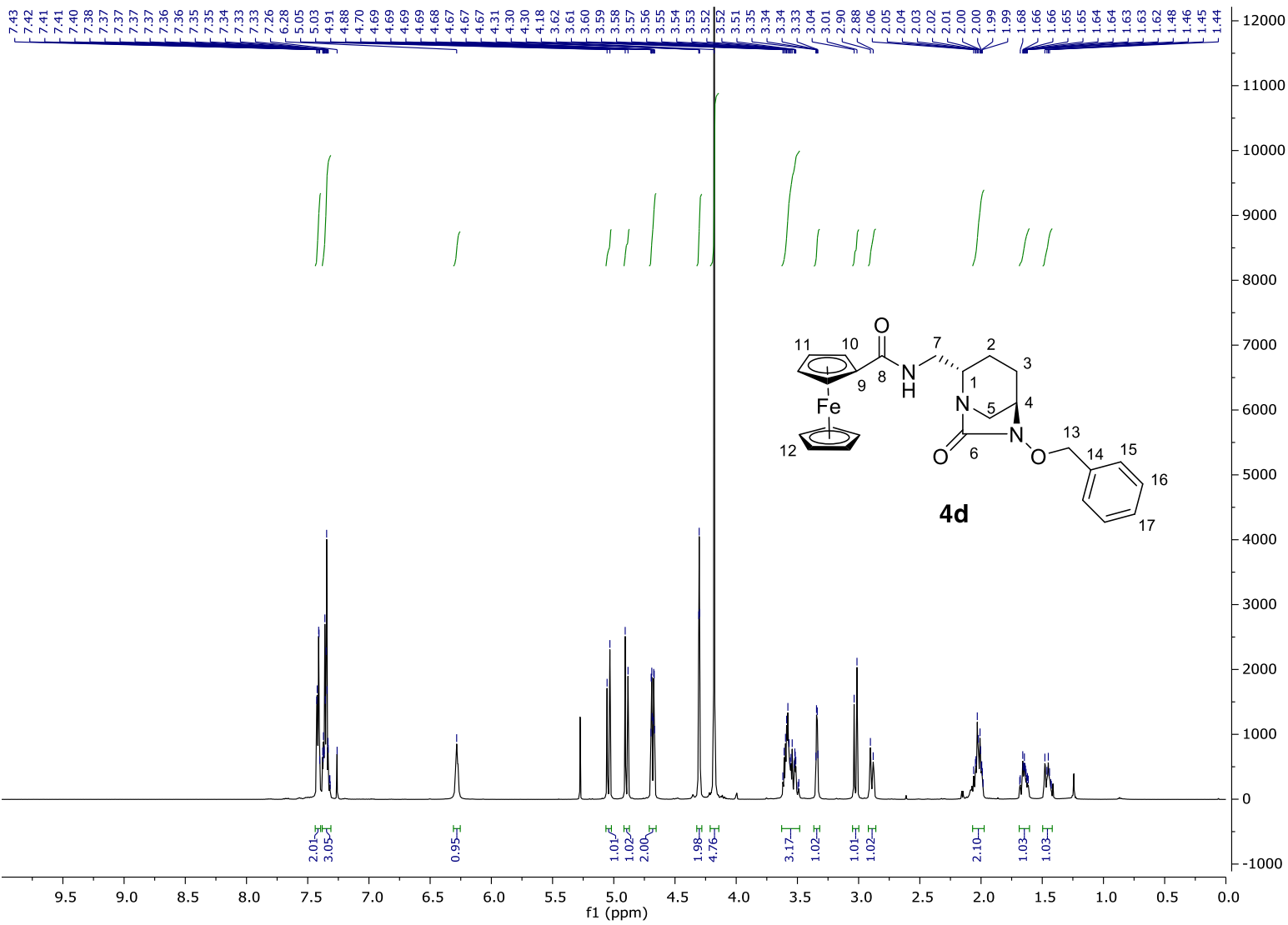

${ }^{13} \mathrm{C} \mathrm{NMR}\left(\mathrm{CDCl}_{3}, 126 \mathrm{MHz}\right)$

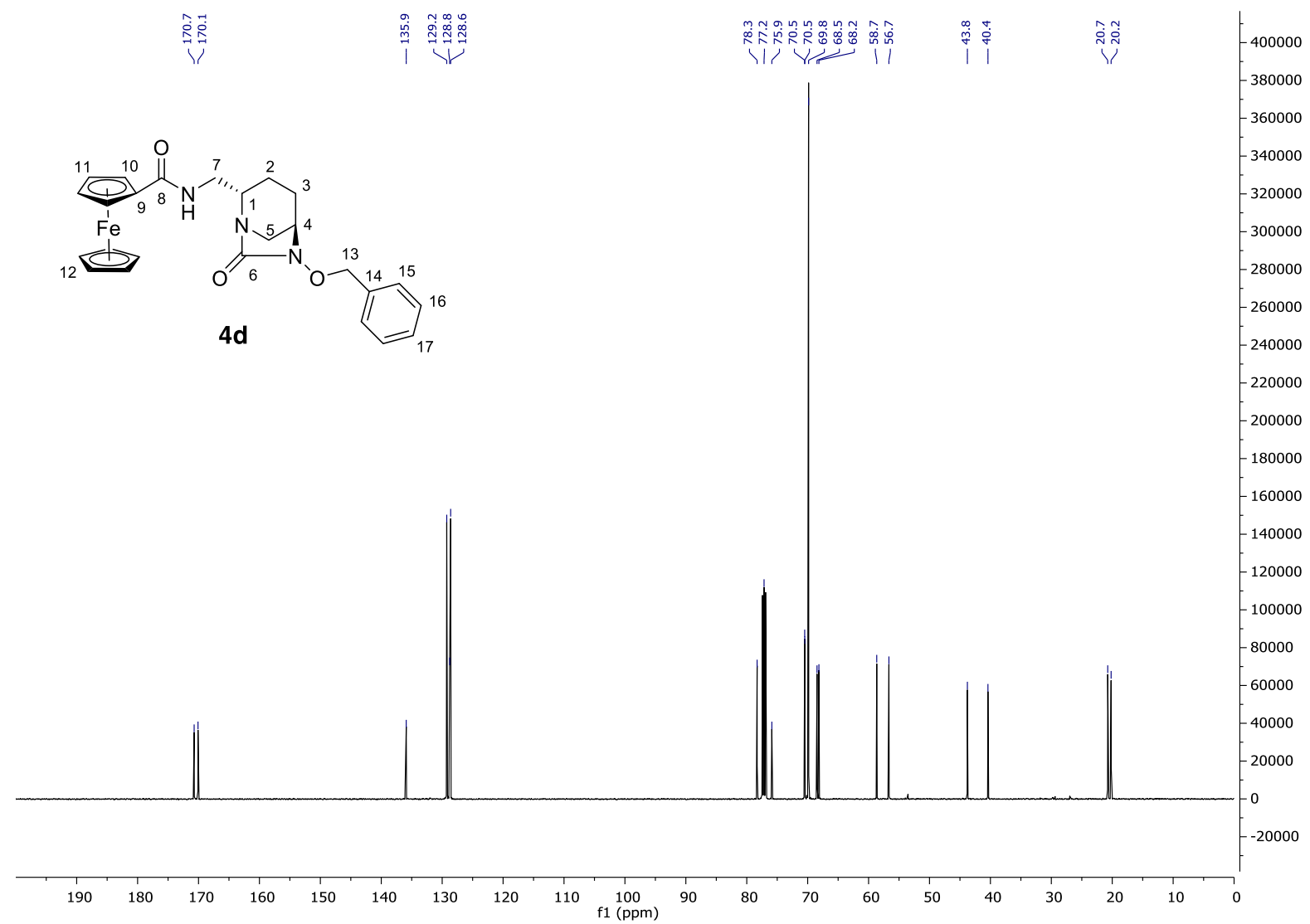


${ }^{1} \mathrm{H}$ NMR $\left(\mathrm{CDCl}_{3}, 500 \mathrm{MHz}\right)$

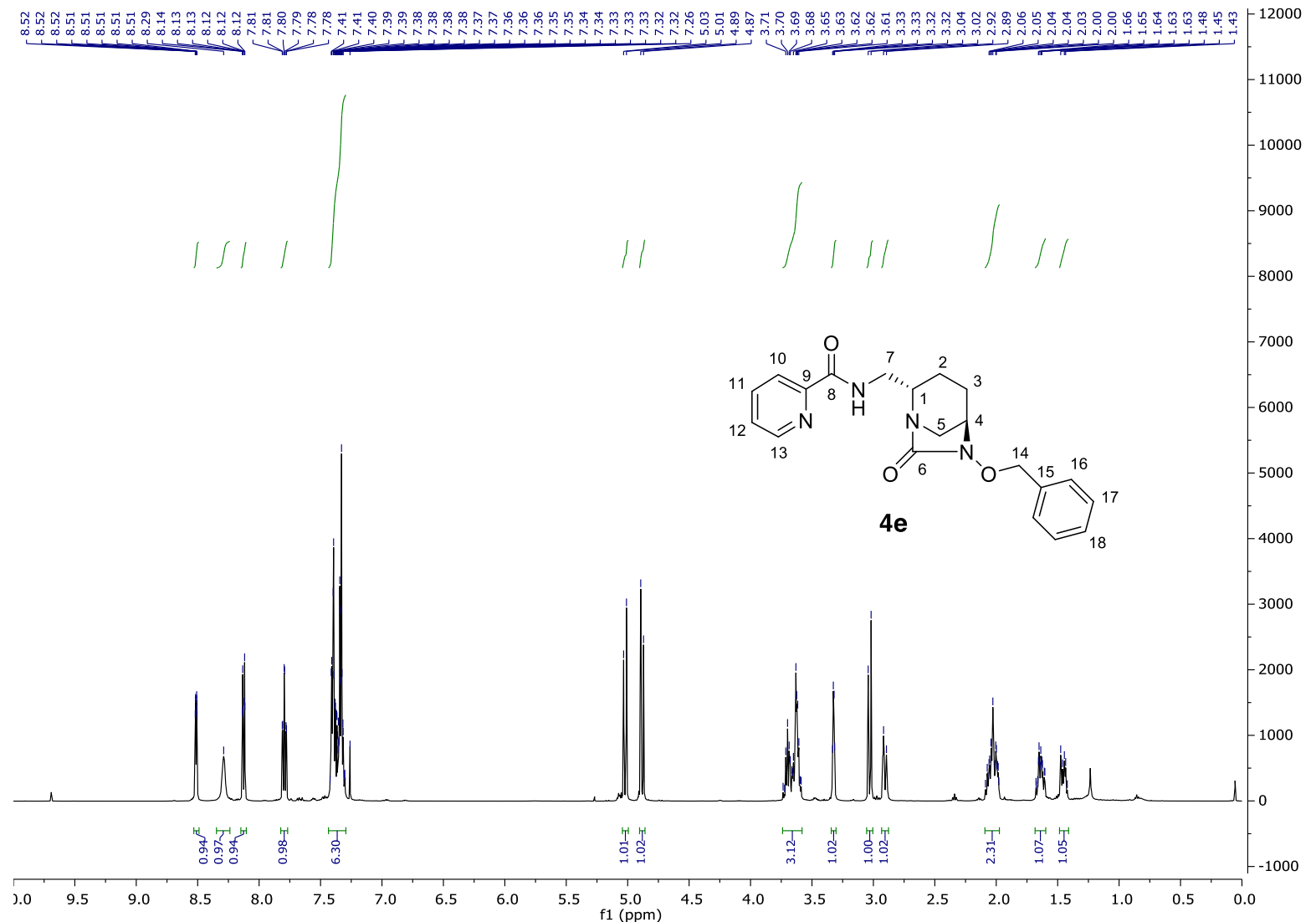

$\left.{ }^{13} \mathrm{C} \mathrm{NMR} \mathrm{(CDCl} 3,126 \mathrm{MHz}\right)$

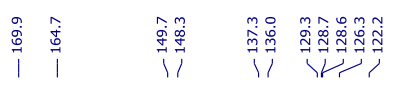

管

$-400000$

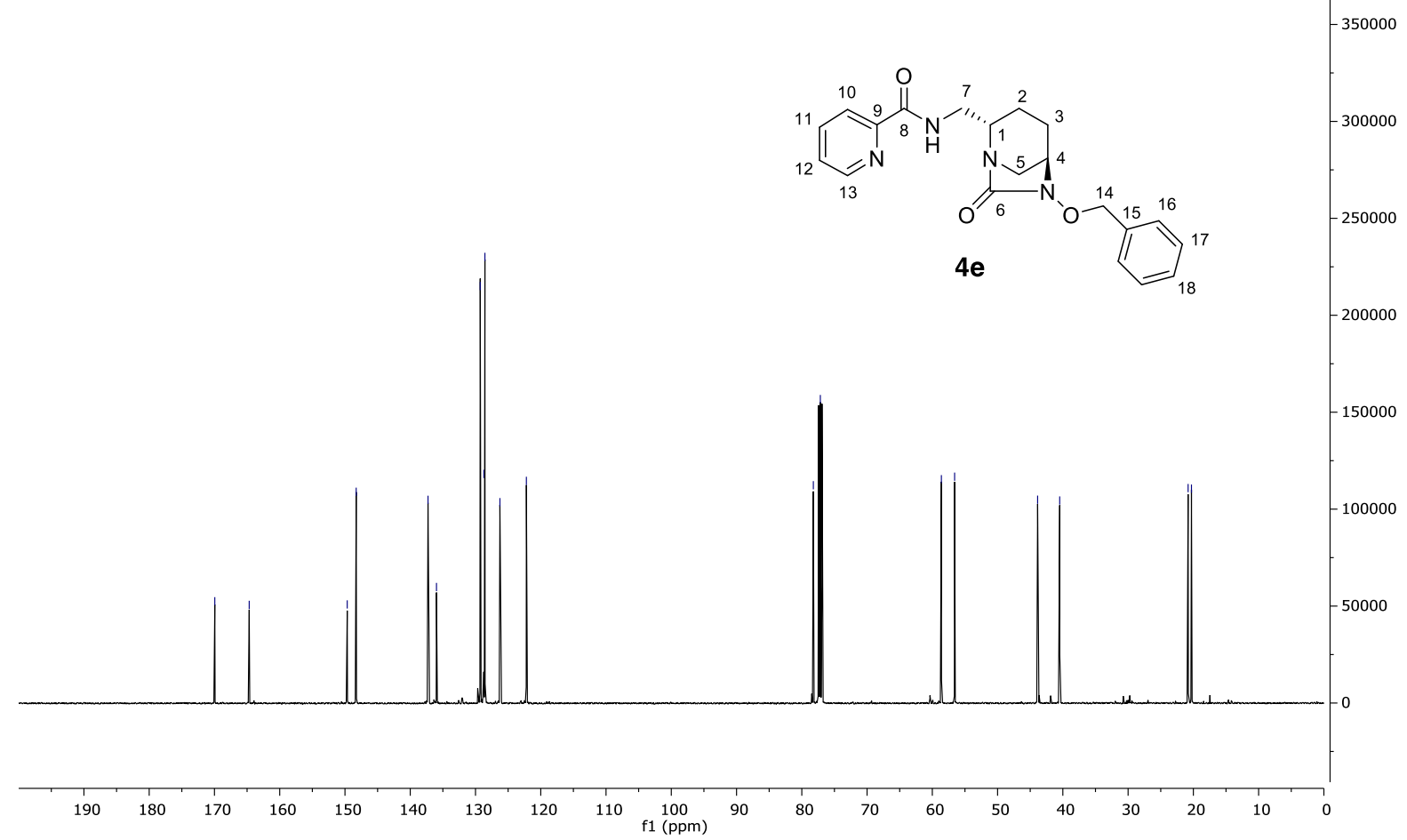




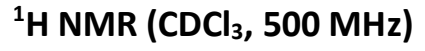

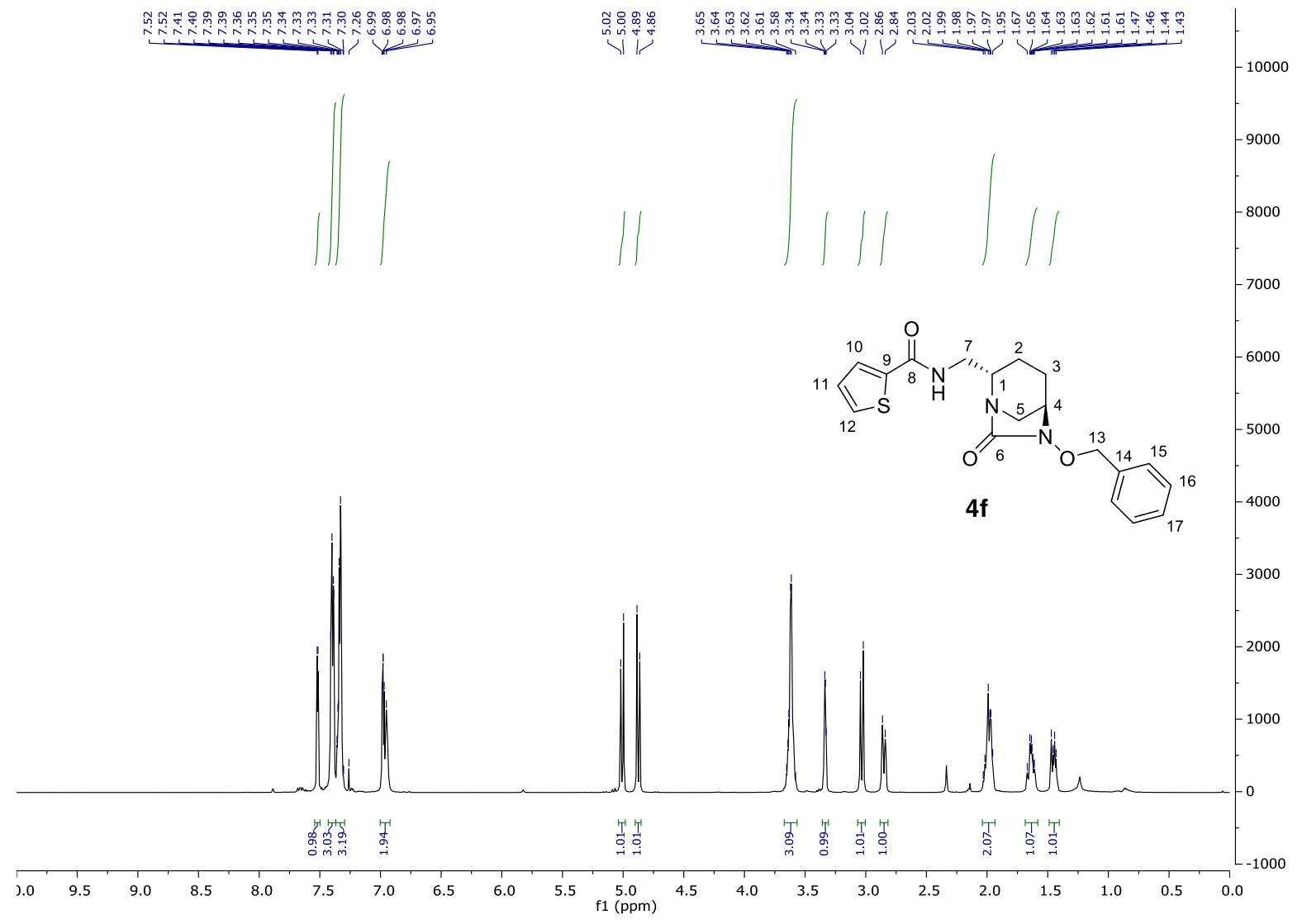

$\left.{ }^{13} \mathrm{C} \mathrm{NMR} \mathrm{(CDCl}, 126 \mathrm{MHz}\right)$

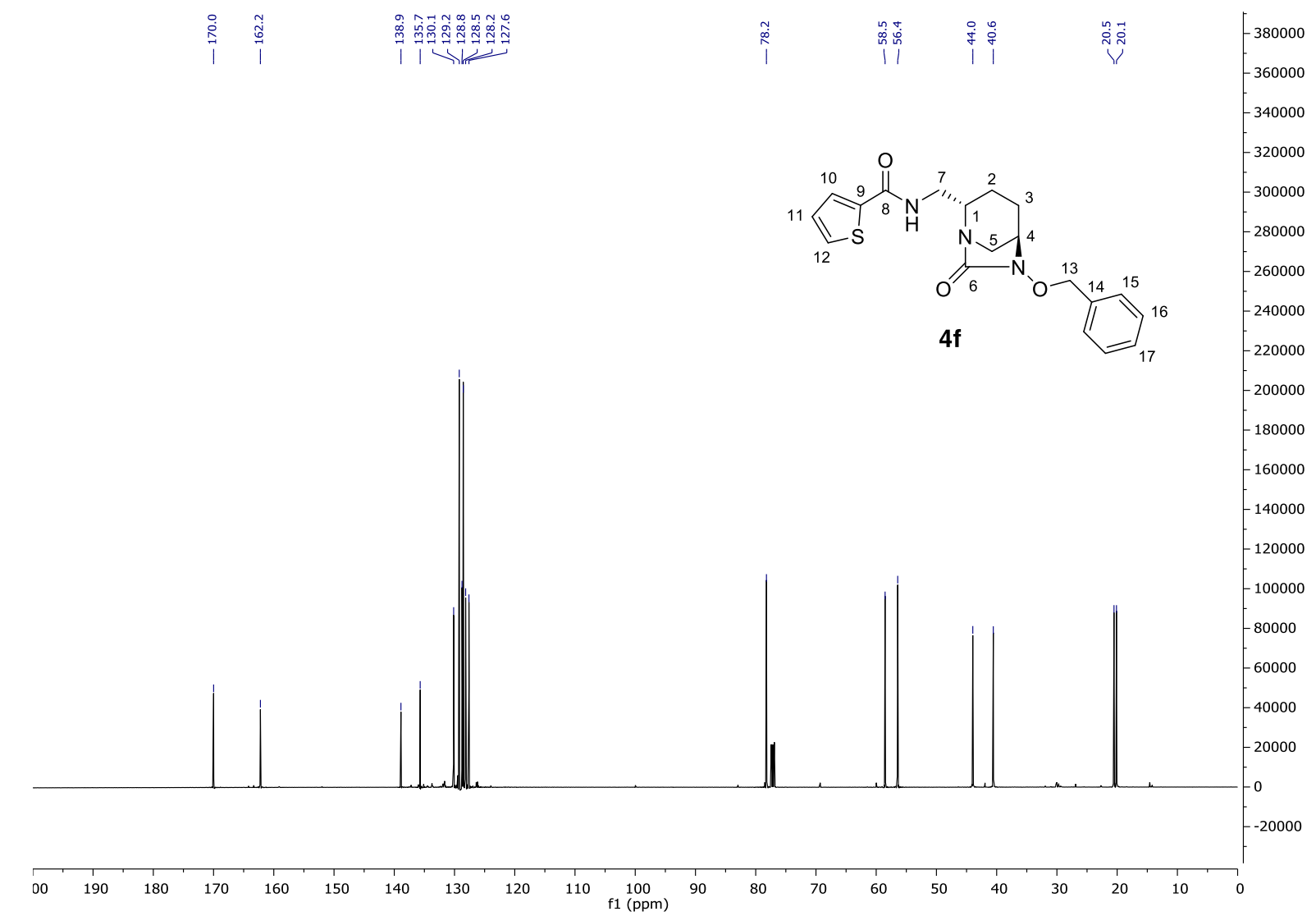


${ }^{1} \mathrm{H}$ NMR $\left(\mathrm{CDCl}_{3}, 500 \mathrm{MHz}\right)$

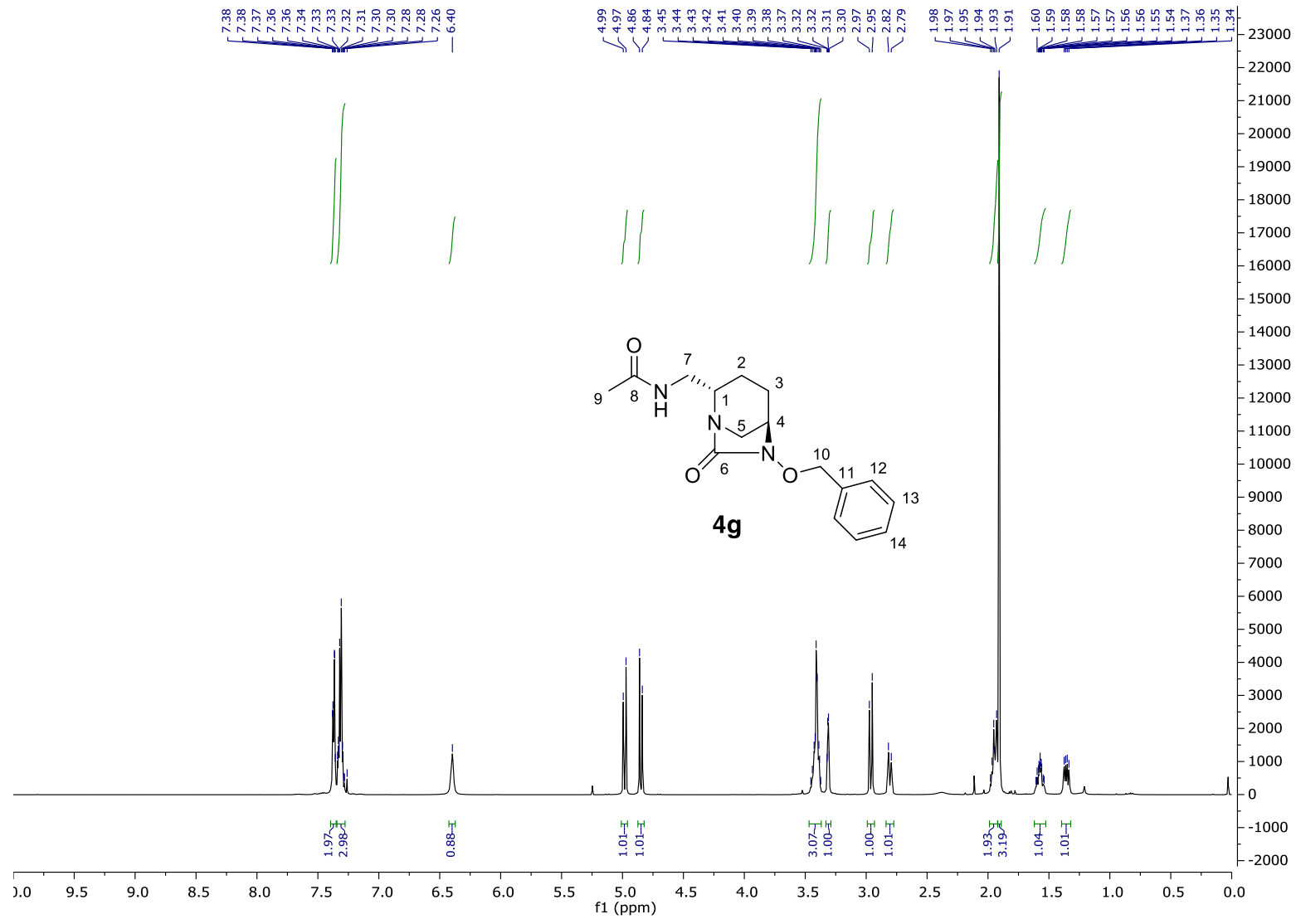

$\left.{ }^{13} \mathrm{C} \mathrm{NMR} \mathrm{(CDCl} 3,126 \mathrm{MHz}\right)$

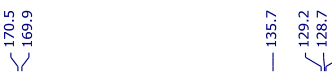

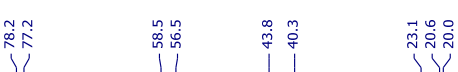

$-450000$

i.

400000
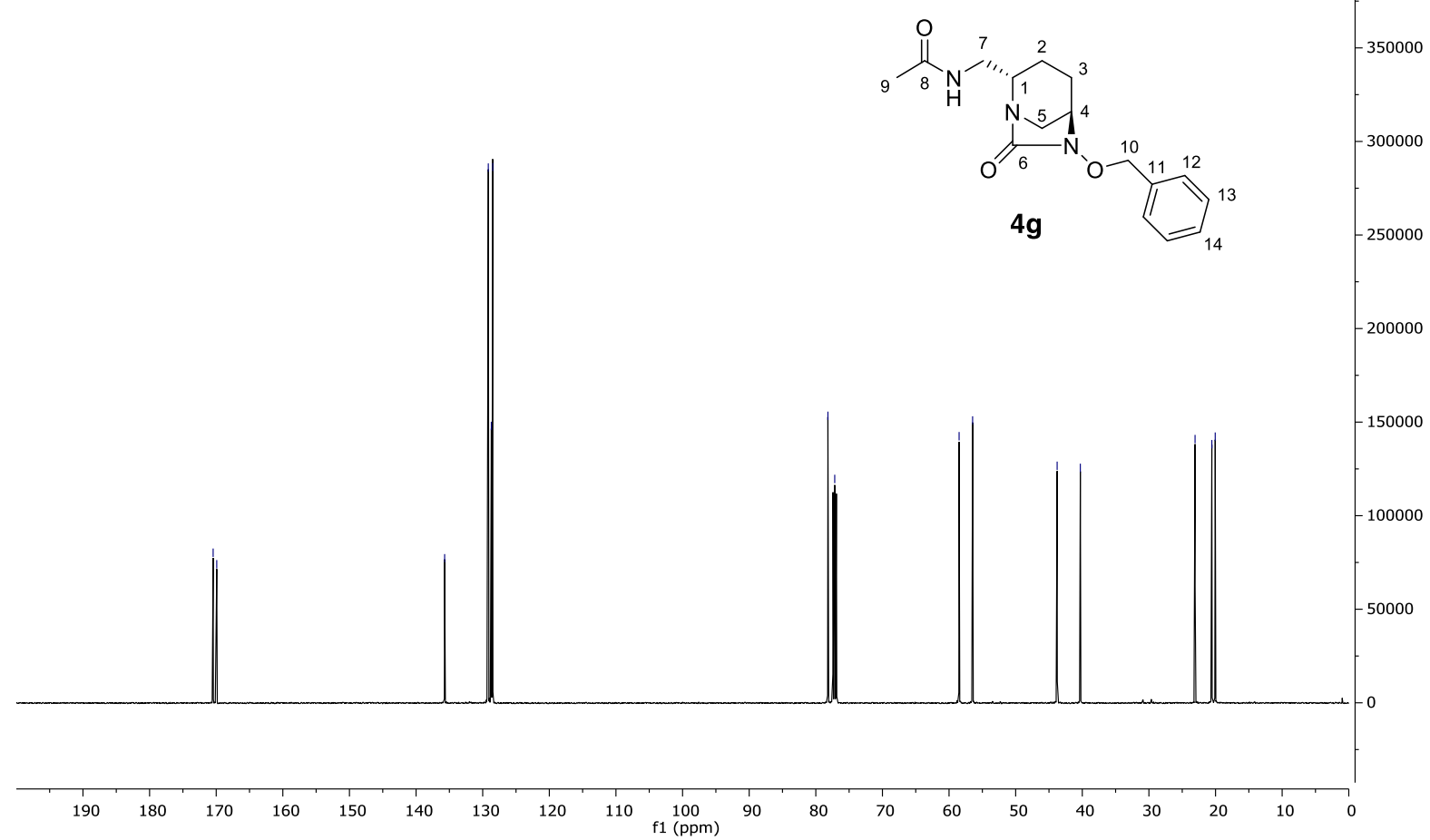

350000 
${ }^{1} \mathrm{H} \mathrm{NMR}\left(\mathrm{CDCl}_{3}, 500 \mathrm{MHz}\right)$

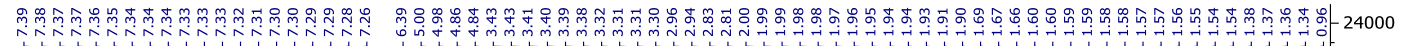
-23000

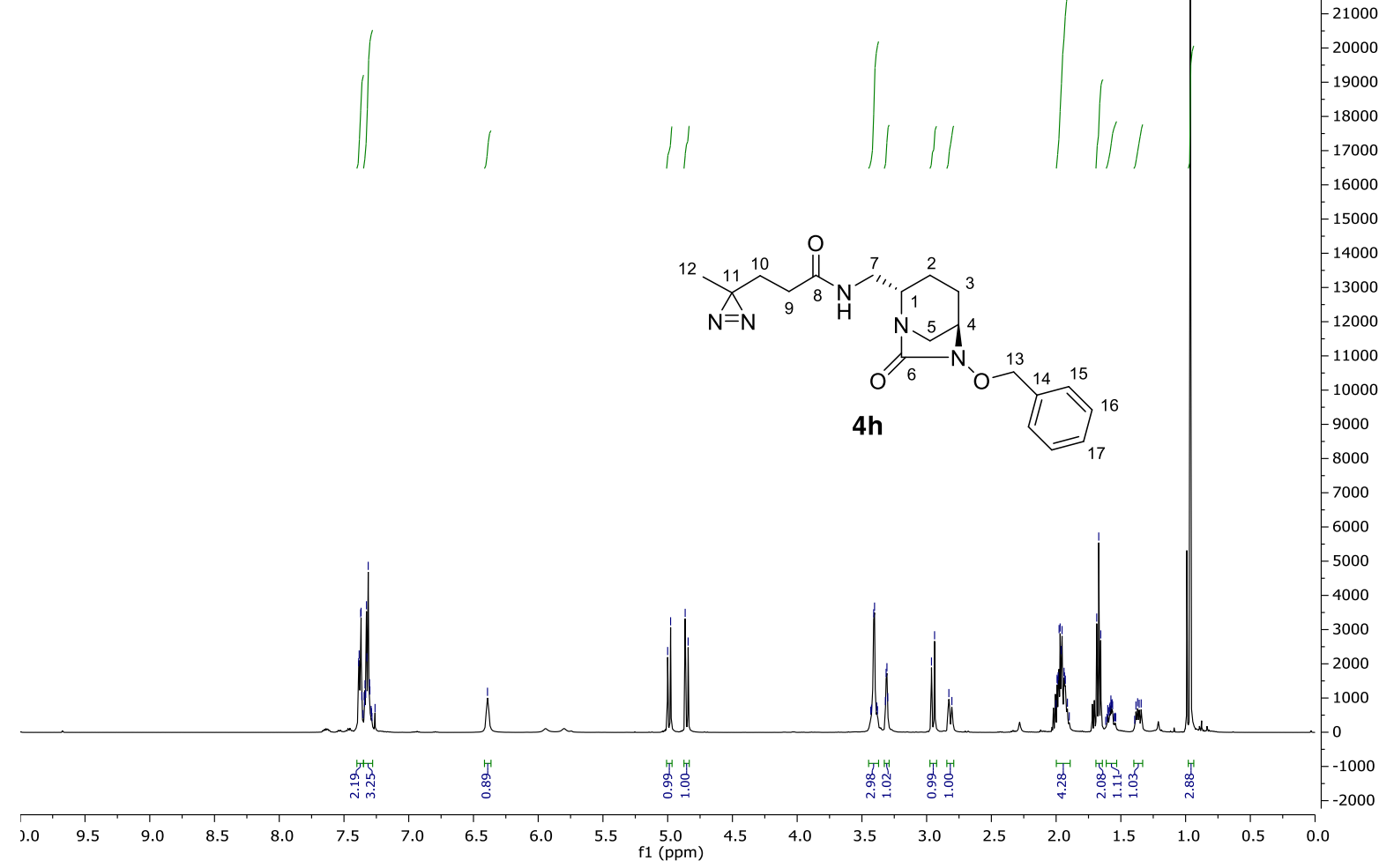

$\left.{ }^{13} \mathrm{C} \mathrm{NMR} \mathrm{(CDCl} 3,126 \mathrm{MHz}\right)$

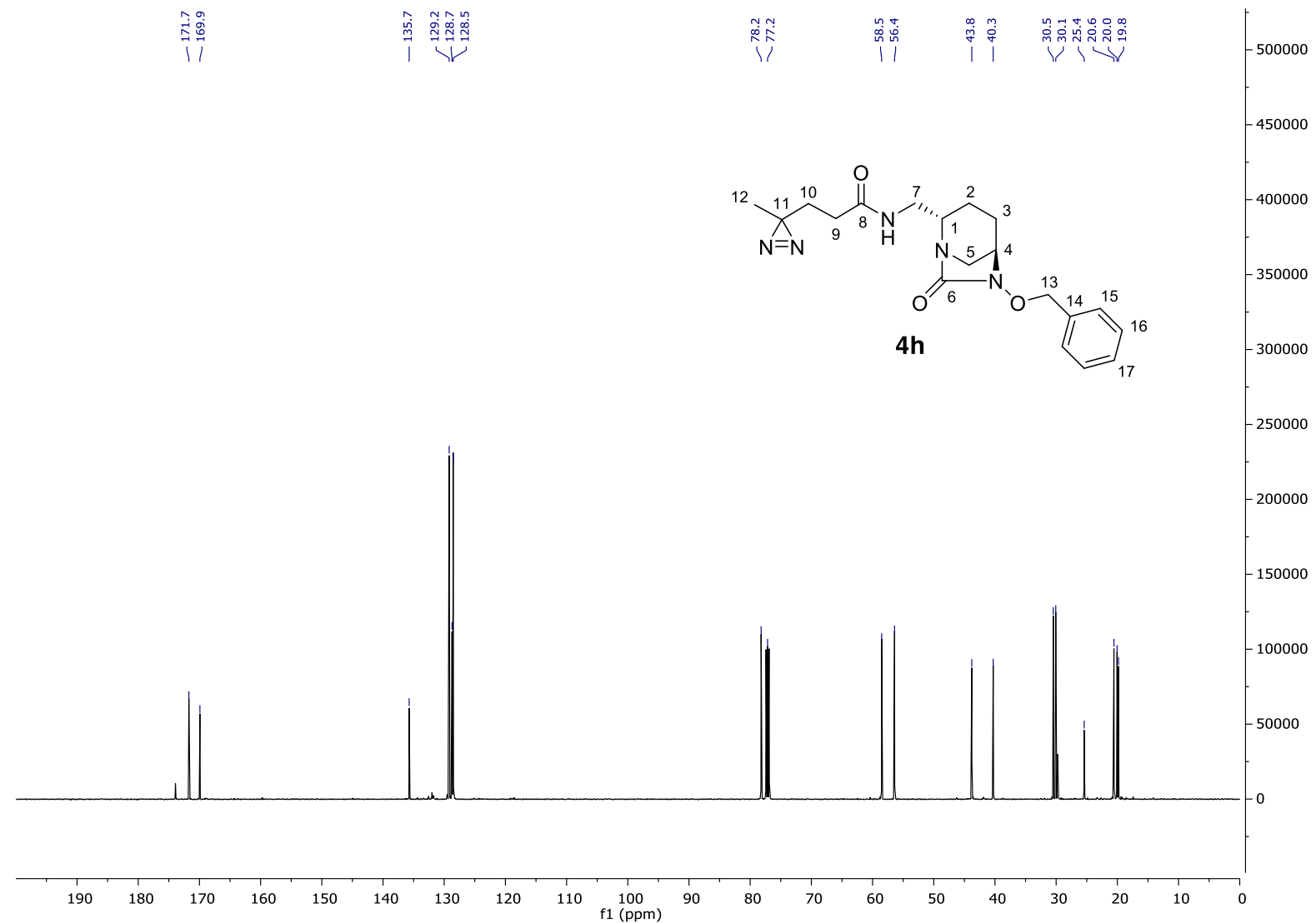


${ }^{1} \mathrm{H} \mathrm{NMR}\left(\mathrm{CDCl}_{3}, 500 \mathrm{MHz}\right)$

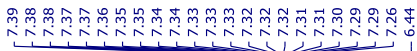

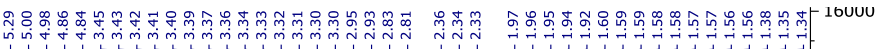
1

\section{0}

14000

13000

12000

/ 11

II

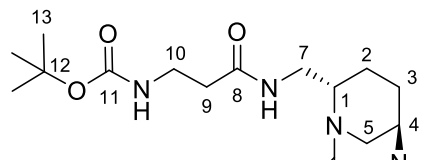

$4 \mathbf{i}$

$\mathrm{O}^{6} \overbrace{18}^{15}$

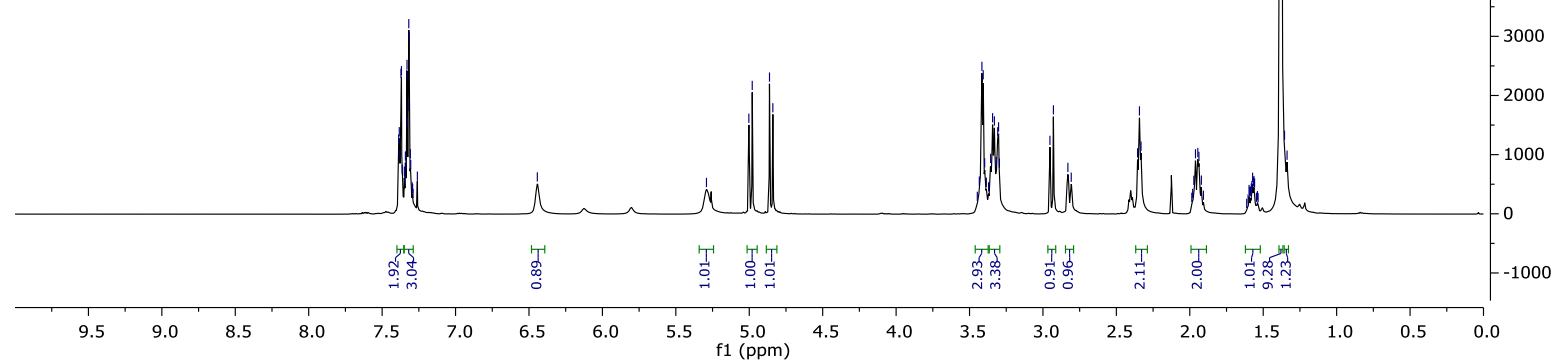

$\left.{ }^{13} \mathrm{C} \mathrm{NMR} \mathrm{(CDCl} 3,126 \mathrm{MHz}\right)$

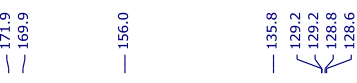

$4 \mathbf{i}$

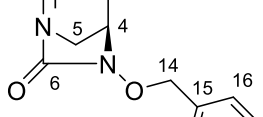<smiles>C1=CC=[In]C=C1</smiles>

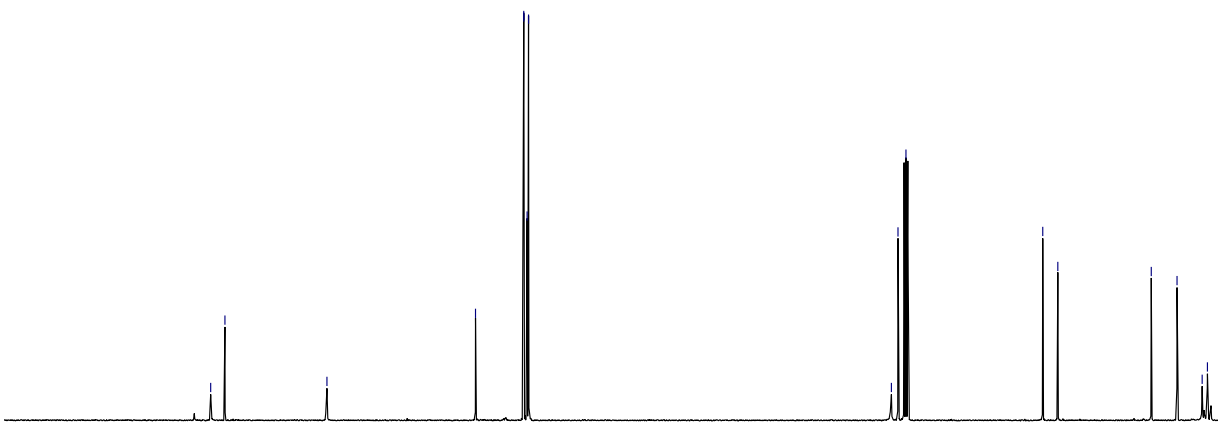

0000

000

0000

0000 


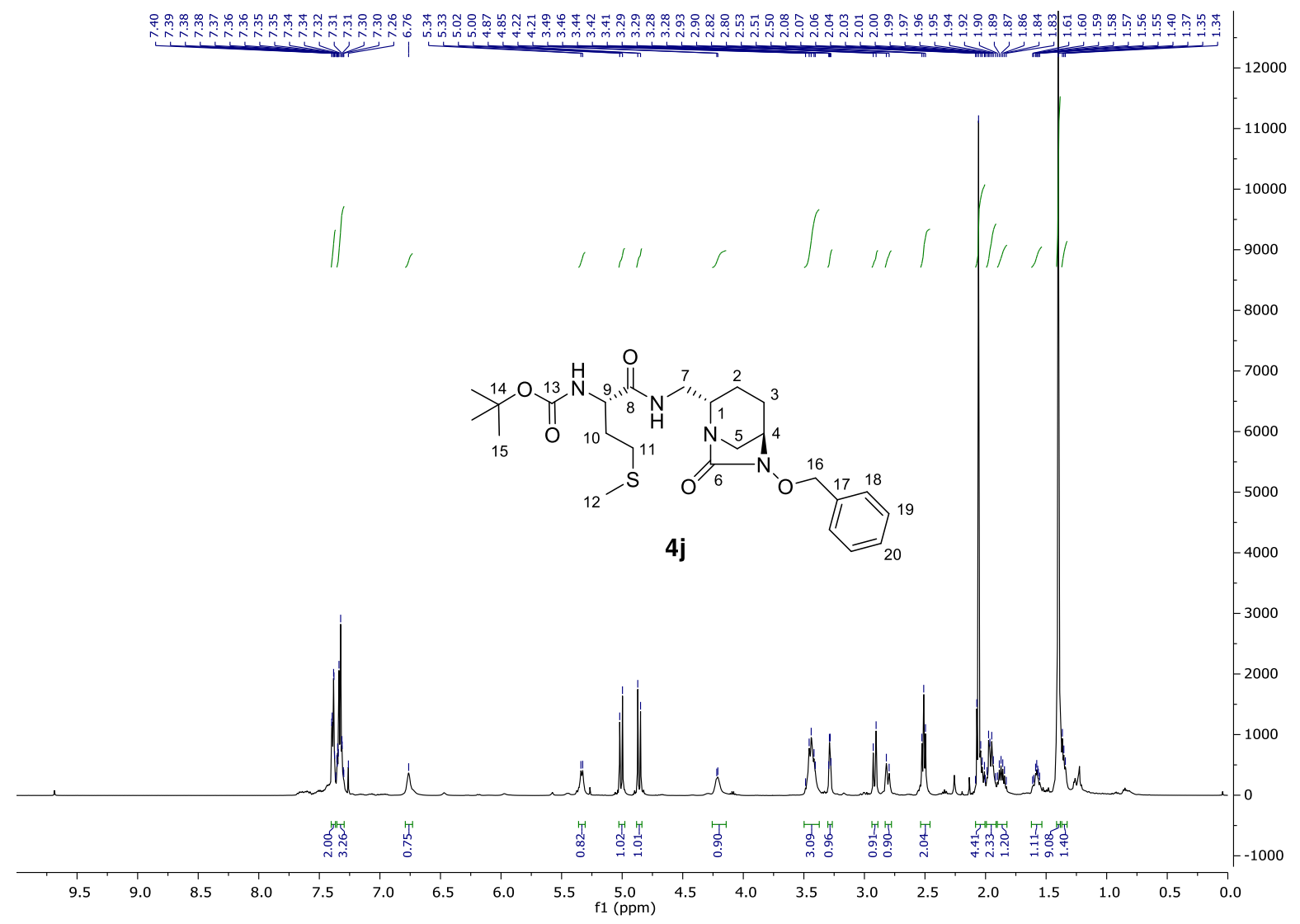

$\left.{ }^{13} \mathrm{C} \mathrm{NMR} \mathrm{(CDCl}, 126 \mathrm{MHz}\right)$

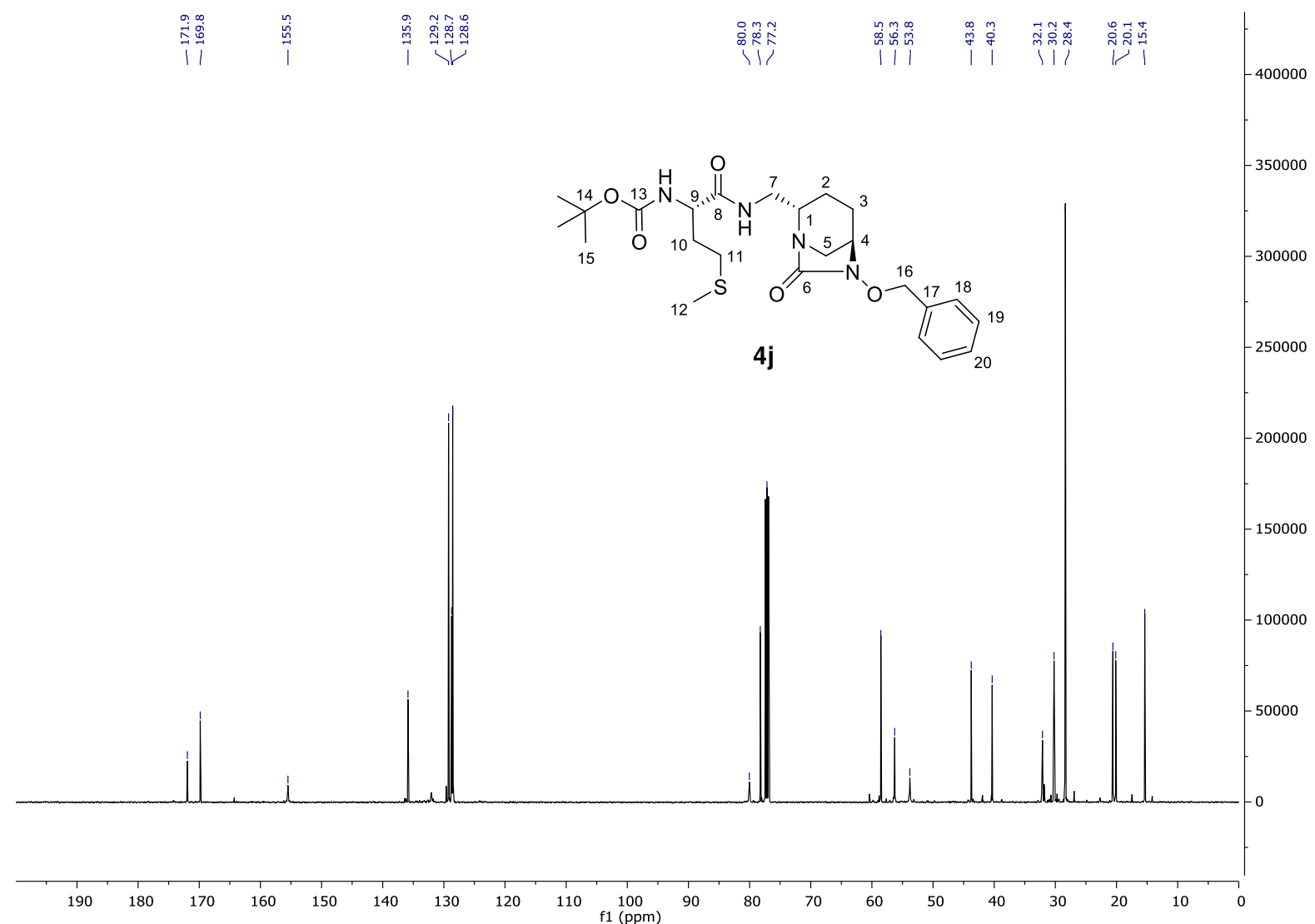


${ }^{1} \mathrm{H}$ NMR (D $\left.20,500 \mathrm{MHz}\right)$

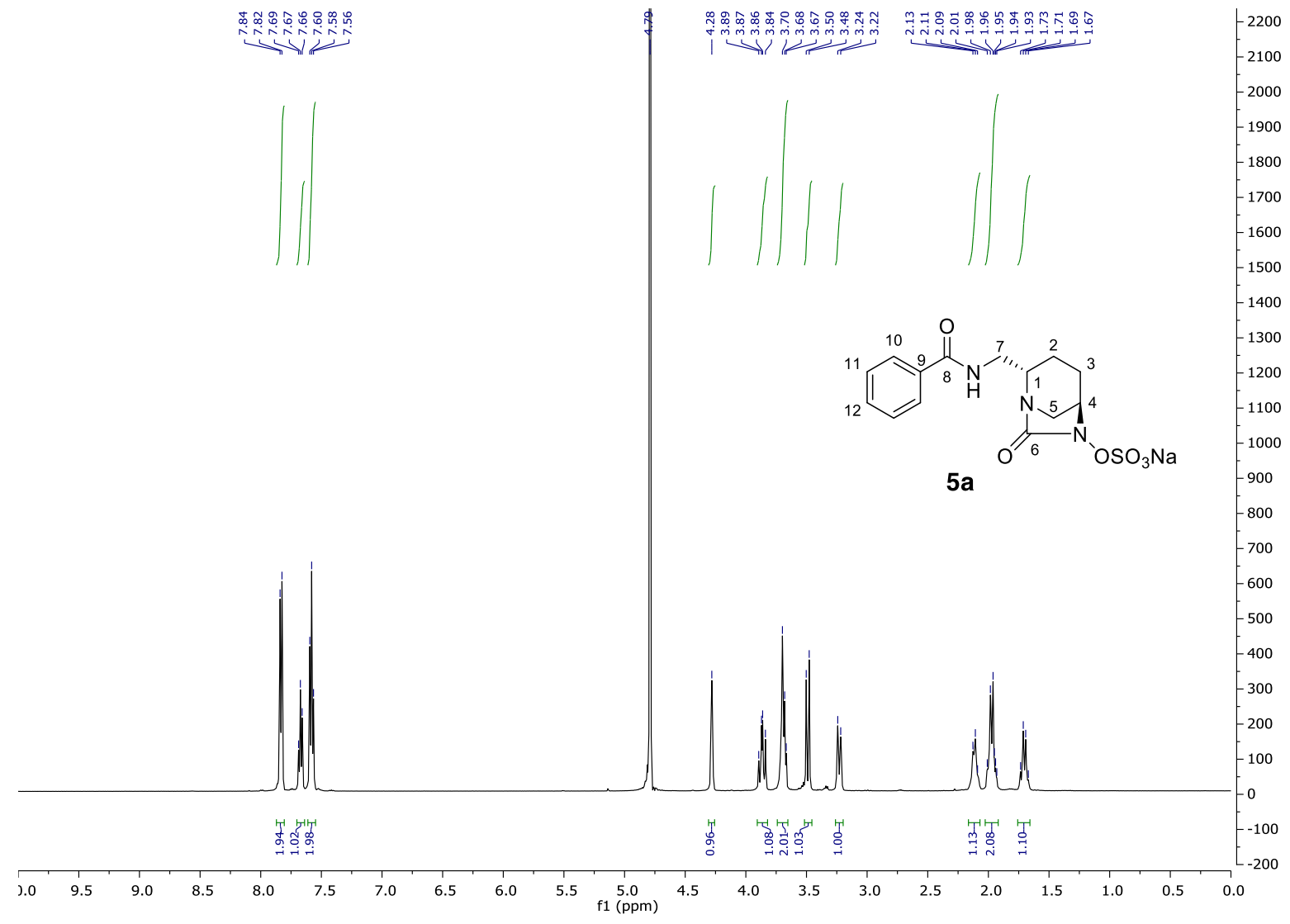

${ }^{13} \mathrm{C}$ NMR $\left(D_{2} \mathrm{O}, 500 \mathrm{MHz}\right)$

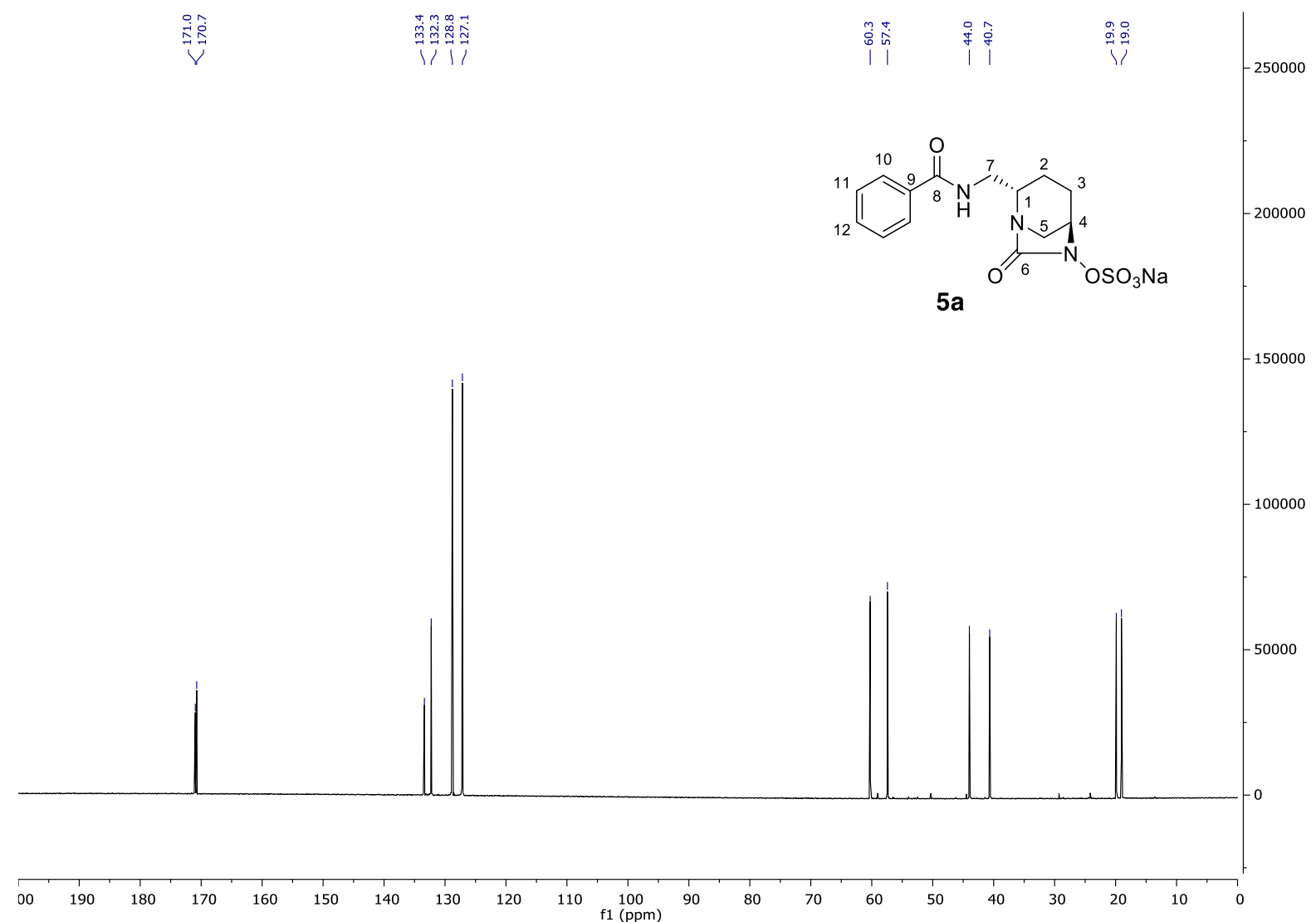




\section{Analytical HPLC}

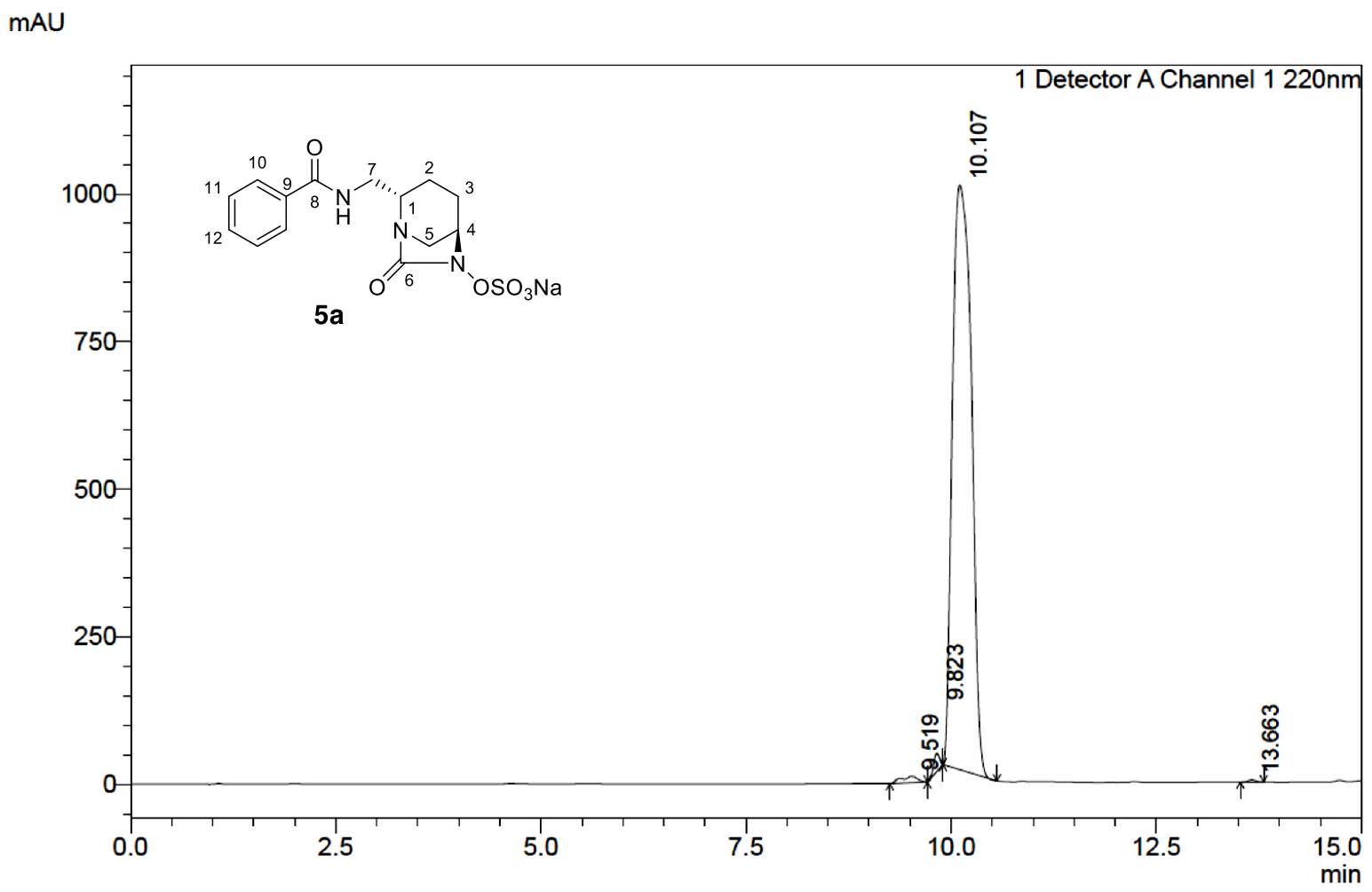

\begin{tabular}{|r|r|r|r|r}
\hline Peak\# & Ret. Time & \multicolumn{1}{|c|}{ Area } & Height & \multicolumn{1}{c}{ Conc. } \\
\hline 1 & 9.519 & 148972 & 10512 & 0.926 \\
\hline 2 & 9.823 & 155303 & 30487 & 0.966 \\
\hline 3 & 10.107 & 15759567 & 990104 & 97.990 \\
\hline 4 & 13.663 & 19028 & 3017 & 0.118 \\
\hline Total & & 16082869 & 1034120 & \\
\hline
\end{tabular}

HYPERSIL ${ }^{\circledR}$ BDS C18 column $(150 \times 4.6$ mm, $5 \mu \mathrm{m})$

Gradient: $\quad 0$ to $100 \% \mathrm{~B}$ in $15 \min \left(\mathrm{A}: \mathrm{H}_{2} \mathrm{O}, \mathrm{B}: \mathrm{CH}_{3} \mathrm{CN}\right)$

Flow: $\quad 1.3 \mathrm{~mL} / \mathrm{min}$

Detection: $\quad 220 \mathrm{~nm}$ 